\title{
DETECTING SUSPICIOUS BEHAVIOR WITH LOW-COST SENSORS
}

\author{
A Thesis \\ Presented to \\ the Faculty of California Polytechnic State University, \\ San Luis Obispo
}

In Partial Fulfillment

of the Requirements for the Degree

Master of Science in Computer Science

by

Ahren Alexander Reed

November 2011 
(C) 2011

Ahren Alexander Reed

ALL RIGHTS RESERVED 


\section{COMMITTEE MEMBERSHIP}

TITLE: Detecting Suspicious Behavior With Low-cost Sensors

AUTHOR: Ahren Alexander Reed

DATE SUBMITTED: November 2011

COMMITTEE CHAIR: Clark S. Turner, Ph.D.

COMMitTeE MEMBER: Neil C. Rowe, Ph.D.

COMMITTEE MEMBER: Gene Fisher, Ph.D. 


\section{ABSTRACT \\ Detecting Suspicious Behavior With Low-cost Sensors}

Ahren Alexander Reed

A proof of concept is created that demonstrates how low-cost sensors and a simple software solution can be used to proactively detect IED placement. The main goal is to detect suspicious behavior; Specifically we derive requirements that loitering, meandering, improper location and object placement shall be detected. Current methods being used to detect Improvised Explosive Devices (IEDs) are costly in terms of equipment and risk to life, and many are retroactive; IED detection occurs long after explosives are placed. A prototype system is explored with the quality attributes of being low-cost, proactive and using simple software methods. A wireless sensor network of simple sensors may alert authorities to people in the act of placing IEDs. Previous work with Crossbow Motes showed that a network of infrared motion sensors can be used to detect loitering. In this prototype nine other sensors are reverse engineered to determine their true operating specifications. Then a prototype sensor network is developed to explore which low-cost sensors can be used to detect suspicious behavior. The results indicate that five low-cost sensors are effective in detecting suspicious behavior: infrared motion, infrared distance, light, force sensors and pressure sensors meet our requirements. 


\section{ACKNOWLEDGMENTS}

This work was supported by the U.S. National Science Foundation under grant 0729696 of the EXP Program. The opinions expressed are those of the author and do not reflect those of the U.S. Government.

Experiments were performed at the U.S. Naval Postgraduate School in Monterey, California, and at the Fort Ord Public Lands in Marina, California.

Permission was obtained by the Computer Science Department at NPS before video, photographs and various non-imaging sensor information was gathered on NPS property for use in this experiment. I am grateful to the NPS Computer Science Department for their gracious assistance and professional administrative support during my summers there as a Research Assistant in 2009 and 2010.

The Fort Ord Public Lands area is open for Day Use by the public. Appreciation goes out to the Bureau of Land Management and the City of Marina Police Department for their dedicated custodianship of the area.

The human subject used for data gathering and observation by sensors was Ahren A. Reed, (myself.) All observed behavior was scripted and used for experimental purposes only.

There is no classified material in this Master's Thesis. All work is purely scientific for academic purposes. All data gathering was conducted with off-the-shelf computer equipment publicly available. All sources cited are available at time of writing through a university library or the Internet (such as IEEE Xplore, and ACM Portal) and no confidential or restricted resources were used in data gathering.

I would like to thank Drs. Clark Turner, Neil Rowe and Gene Fisher for their participation in this thesis and for their mentorship. 


\section{Contents}

List of Tables $\quad$ viii

List of Figures $\quad$ ix

1 Introduction to the problem space 1

1.1 Thesis Statement and Quality Attributes . . . . . . . . . . . . . 2

1.2 Definition of suspicious behavior and Derived Requirements . . . . . . . . . . . 5

2 Related Work $\quad 10$

3 Previous Work $\quad 15$

3.1 Research with IR motion sensors . . . . . . . . . . . . . . . . . . . 15

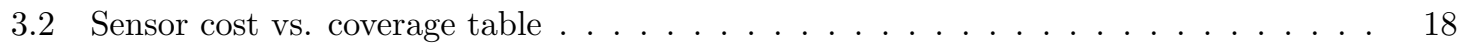

4 Experiment Design $\quad 24$

4.1 Black-box testing of sensors (see appendix A for experiment date \& charts) . . . . 24

4.1 .1 Preliminary Experiments _. . . . . . . . . . . . . . . . . . . . 29

4.1.2 Reverse engineering sensors to determine specifications . . . . . . . . . . . . 32

$4.1 .3 \quad$ Physical Contact Sensors _. . . . . . . . . . . . . . . . . . 34

4.1 .41111 Infrared Motion Sensor _ . . . . . . . . . . . . . . . . . 36

4.1 .5 Light Sensor $1127 \ldots \ldots \ldots \ldots \ldots$. . . . . . . . . . . . . . 37

4.1.6 Sonar Distance Sensor $1128 \ldots \ldots$. . . . . . . . . . . . . . . . . . 39

4.1.7 Phidgets 1101 IR Distance Adapter and 3521 Infrared Distance Sensor . . . . 41

4.1 .8 Phidgets 1108 Magnetic Sensor . . . . . . . . . . . . . . . . . . . . . . . . 42

4.2 Prototype testing in field (see appendix B for experiment data \& charts) . . . . . . 44 
4.2.1 Experiment in Quad, proof of concept . . . . . . . . . . . . . . 44

4.2.2 Experiment Plan . . . . . . . . . . . . . . . . . . . . 47

4.2 .3 Analysis of sensors . . . . . . . . . . . . . . . . . . . . . . 48

4.2.4 Quick summary of sensor performance . . . . . . . . . . . . . . 54

5 Conclusion and future work $r 56$

5.1 Strengths and Weaknesses of this Work . . . . . . . . . . . . . . . . 56

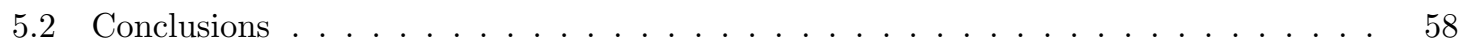

5.3 Future work . . . . . . . . . . . . . . . . . . . . . . . . 59

$\begin{array}{lr}\text { A Phidgets black box testing } & 66\end{array}$

B Quad experiments analysis $\quad 104$

B.1 Infrared Motion Sensor Analysis . . . . . . . . . . . . . . . . . . . . . . . . . . . . 104

B.2 Infrared Distance Sensors . . . . . . . . . . . . . . . . . . . . . . . . 115

B.3 Sonar Distance Sensors . . . . . . . . . . . . . . . . . . . . . . . . . . . 125

B.4 Light Sensors . . . . . . . . . . . . . . . . . . . . . . . . . . 135

$\begin{array}{lr}\text { C False Positives of Sensors } & 138\end{array}$

$\begin{array}{lr}\text { D Wireless Sensor Networks } & 139\end{array}$

E Prototype sensor network proposal 142

F Computer Science Methodology 144

$\begin{array}{lr}\text { G Phidgets data acquisition code } & 148\end{array}$ 


\section{List of Tables}

2.1 Overall results comparing related work . . . . . . . . . . . . . . . . . . . . 14 


\section{List of Figures}

1.1 derived requirements will categorize different types of suspicious behavior . . . . . . 4

1.2 derived requirements used in this thesis $\ldots \ldots \ldots \ldots \ldots$

3.1 Tracking a single subject with Crossbow Motes . . . . . . . . . . . . . . . . . . 22

4.1 Developing metrics for derived requirements . . . . . . . . . . . . . . . . . . 24

4.2 Normal activity measured in Glasgow Quad . . . . . . . . . . . . . . . . . 26

4.3 Suspicious activity (loitering) measured in Glasgow Quad . . . . . . . . . . . . . . 27

4.4 Linking metrics to derived requirements $\ldots \ldots \ldots \ldots \ldots$

4.5 Glasgow Hall Quad image courtesy N. Rowe . . . . . . . . . . . . . . . . . . . . . . 29

4.6 Tracked paths through Glasgow Hall Quad image courtesy N. Rowe . . . . . . . . . 29

4.7 Likely IED placement in Glasgow Hall Quad image courtesy N. Rowe . . . . . . . . 30

4.8 The MaxBotix EX-1 sonar sensor field of vision . . . . . . . . . . . . . . . . . 39

4.9 placement of sensors in Glasgow Hall quad . . . . . . . . . . . . . . . . . . . . 49

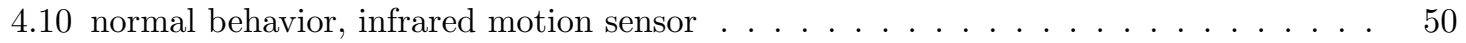

4.11 suspicious behavior detected with infrared motion sensor . . . . . . . . . . . . . 51

4.12 Normal behavior, tracking an individual walking . . . . . . . . . . . . . . 52

4.13 suspicious behavior, object left in front of sensor . . . . . . . . . . . . . . 52

4.14 noisy sonar sensors reporting false positives $\ldots \ldots \ldots \ldots \ldots$

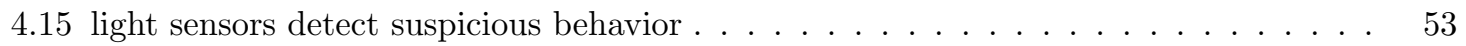

5.1 developing links between sensors and detecting types of suspicious behavior . . . . . 59 


\section{Chapter 1}

\section{Introduction to the problem space}

The problem space surrounding this thesis is the chaotic world surrounding improvised explosive devices (IEDs) and terrorism. IEDs kill many people and don't discriminate between civilians and soldiers. Statistics from NATO's International Security Assistance force in Afghanistan show that in 1999, 149 ISAF soldiers and 435 civilians were killed [13]. Civilians who are not involved in the war are being killed during day to day activities such as driving to work, more frequently than soldiers are being killed. With respect to military fatalities in the theater, the percentage of soldiers killed in action by IEDs runs between 60 and 75 percent, compared to other combat-related deaths.

There are four main types of IED:

- Package type (trashcan bomb)

- Vehicle (car bomb)

- Carried by human being (suicide bomb)

- Buried on path or road (roadside bomb)

For example, an explosive charge may be placed in a bicycle pannier bag, such as the one that killed the chairman of Deutsche Bank on Nov. 30, 1989 [22]. A shaped charge sent a 2 kg. slug of molten copper through the passenger door of his Mercedes. One could consider that a "package bomb" yet there are others. There is a whole industry devoted to manufacturing bomb-proof waste receptacles for public spaces. A bomb connected to a cell-phone trigger can be tossed easily into a 
trash can at the farmers' market and detonated remotely. A bomb-proof container that weighs 1000 lbs will direct the blast upward and away from nearby pedestrians. Suicide bombers regularly board buses in Tel Aviv with the goal of detonating themselves on a packed bus [6]. This thesis focuses on the problem space around roadside bombs, that is, explosive devices buried or otherwise concealed in a public place.

Roadside IEDs can look like anything. They can be grenades piled together under pieces of cardboard, or mines stacked together and buried in the sand. Often they are unexploded (and perhaps very old and unsafe) tank artillery rounds linked together with a detonator and buried. Some IEDs have been found weighing up to 500 lbs. IEDs are placed on known military patrol routes, concealed in construction debris such as a block of concrete or a pile of garbage.

\subsection{Thesis Statement and Quality Attributes}

Thesis Statement: This thesis asks the question, "Can a simple software system using low cost sensors be used to pro-actively detect suspicious behavior?" We have shown in previous work that Crossbow Motes with infrared motion sensors can track individuals and detect suspicious behavior [32]. Using rapid prototyping, we build a simple software system to test other low-cost sensors for their use in a system to monitor an area and automatically detect suspicious behavior.

\section{Main Quality Attributes}

The goal of this work is to detect suspicious behavior that can lead to IED emplacement. There are three quality attributes the solution focuses on:

- Inexpensive (possible solution: COTS hardware) example: CubeSat [23]

- Simple (can be maintained by non-domain experts)

- Proactive ("The only window of opportunity you have is when they are emplacing the IEDs.") [15]

\section{Inexpensive}

Launching a satellite into space in the 1960's was very expensive. For example, the first communications satellite launched in 1965 was Intelisat I (Early Bird) designed by Hughes. It had an operating 
cost (building, launching and 1.5 year lifespan) of $\$ 15,600,000$, and weighed in at $68 \mathrm{~kg}$ [18]. The current trend is toward reduction of costs to achieve the same benefit. An example is Cal Poly's CubeSat program which uses Commercial Off-the-Shelf (COTS) hardware [23]. New advantages in materials allow researchers to minimize mass and volume. A small communications satellite can be launched into orbit now for around $\$ 40,000$, and weighing in at only $1 \mathrm{~kg}$. [8]

Although COTS military procurement started in 1994 [17] it has become more important in recent years as military budgets have been reduced. COTS hardware is currently being used by the U.S. Navy to lower submarine construction and maintenance costs. The Navy realized it could spend more money on research \& development or simply purchase sonar technology that was available in industry for less [4]. These measures come at the end of what a Houston Chronicle article calls, "the post-9/11 era of blank-check military spending" [3]. United States Secretary of Defense Robert Gates recently spoke to the American Enterprise Institute where he discussed the fiscal crisis and cutbacks in military spending:

Reversing an unsustainable course - where more and more money is consumed by fewer and fewer platforms that take longer and longer to build - meant reforming the acquisition process and the department's buying culture. The goal is that any new weapons system should meet benchmarks for cost, schedule and performance while minimizing 'requirements creep' - the kind of indiscipline that leads to 25 million dollar howitzers, 500 million dollar helicopters, 2 billion dollar bombers, and 7 billion dollar submarines. [12]

\section{Simple Solution}

Hierarchic systems are often nearly decomposable. Hence only aggregative properties of their parts enter into the description of the interactions of those parts. -Herb Simon, The Sciences of the Artificial [35]

The Crossbow MSP410 was a commercial attempt to create a wireless sensor network security. It was ultimately unsuccessful, and one of the reasons may be that customers were frustrated at not

being able to modify the program loaded on the units, (I know I was.) Most MicaZ Motes are entirely user-programmable with NesC; these were meant to be used with a proprietary program only. This work shows the necessity of user-maintained software; the basis of object-oriented programming is 


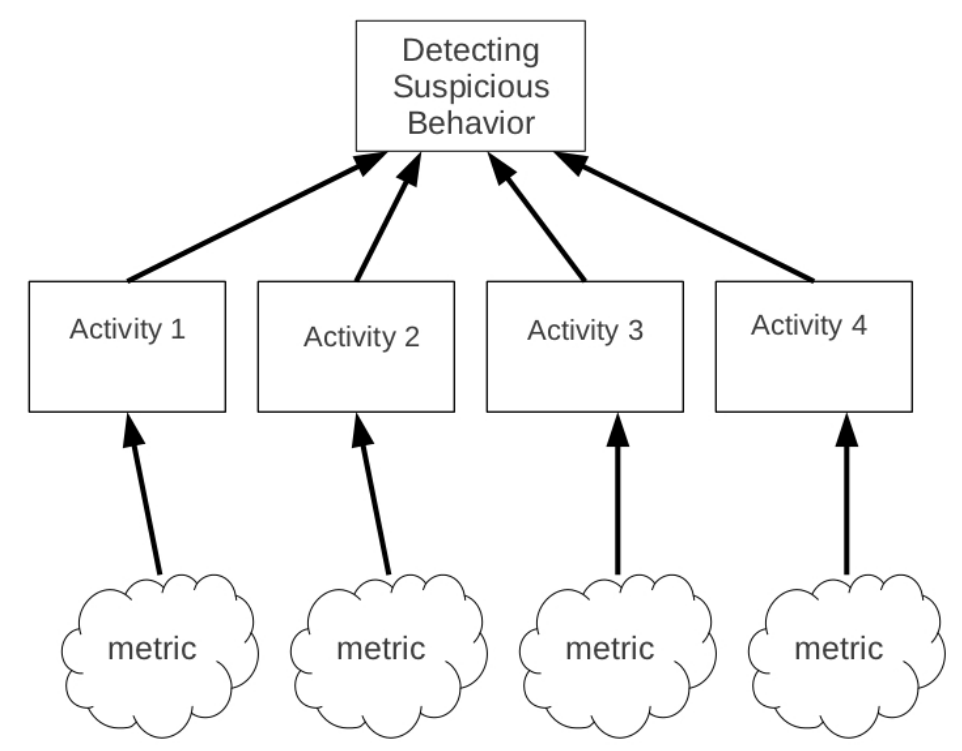

Figure 1.1: derived requirements will categorize different types of suspicious behavior

to be able to add and modify methods to suit the use of the software. Rather than embedding a complex, proprietary algorithm on a chip and packaging it as a one-program-fits-all solution, this work seeks simple solutions in programs and algorithms. This is essential for maintenance and modification of the system by non-experts in the field.

The main requirement of detecting suspicious behavior can be broken into derived requirements which categorize different types of suspicious behavior (loitering, meandering, etc.) A metric is developed for data analysis which tells the observer when one of the derived requirements is being detected. Simple software solutions can be created that analyze that metric from the data gathered. An example of this is writing the software algorithm in Matlab to plot a linear best-fit curve to track a subject walking past IR Motion sensors, as we did in our previous work [32]. Figure 1.1 shows derived requirements are linked to suspicious activities. The activities are measured by simple sensors to detect suspicious behavior. The Metrics determine which sensors and what readings they give.

A modular approach allows the problem of detecting suspicious behavior to be broken into smaller pieces, each with a simple solution. Each time a new derived requirement is found, information can be analyzed and assembled to detect suspicious behavior. For example, we want to detect loitering with a new type of sensor: A metric can be added that analyzes data from this sensor and as- 
sembled into a larger solution to detect suspicious behavior. The metrics themselves may be unique to each kind of sensor and users can implement tools using widely available software to analyze data.

\section{Proactive}

When you find someone placing an IED, you now have the opportunity to gather information [15]

- Where their ammo stash is located

- Who they are working with

- Where other IEDs are placed

The U.S. Military currently deals with roadside bombs after-the-fact. Very little detection is being done as IEDs are being placed. Most of the detection is done after bombs have been placed and cleanup responsibility falls on dedicated Route Clearance Teams and Ordinance Disposal Technicians. Route clearance teams drive attack-and-bomb-resistant vehicles such as the MRAP Buffalo [13]. These vehicles have robotic arms with enormous pitchfork-like tines to sift sand and dig through suspicious piles of debris on the road. It is better to blow a few tines off an expensive robot arm than risk the lives of soldiers. Route clearing teams are effective but slow. It may take three days for a team to clear $70 \mathrm{~km}$ of road. All the while they are being followed by a convoy of delivery vehicles and passenger cars; those are the residents of Afghanistan trying to do their jobs and get to their families. Roadside bombs are more dangerous for the people living in the war zone.

A proactive solution built with sensors to monitor a road will ultimately reduce costs in the long run. More lives can be saved, the danger of ordinance disposal can be reduced or eliminated, and there is less machinery involved.

\subsection{Definition of suspicious behavior and Derived Require- ments}

In order to detect suspicious behavior we must set up a definition for normal behavior that occurs in the environment being monitored. For example, the normal behavior for a suburban street is that cars drive down it at a maximum of $25 \mathrm{mph}$ and sometimes people walk by on the sidewalk. This can 
be used as a comparison against observed behavior once a system is set up to monitor the area, to determine whether or not any new observed behavior is suspicious. If we observing someone walking at night down the sidewalk and suddenly leaving the sidewalk to peer through windows of houses, this indicates suspiciousness. A policeman driving by might think this is a cat-burglar looking for a house to rob. In fact, going by one's gut feeling is sometimes enough to detect suspiciousness! [21] This example simply serves to separate acceptable behavior for the environment from that which is not. Leaving the sidewalk is out of the norm. Peering through windows at night is out of the norm.

At other times, detecting suspicious behavior may not be so obvious. Everyone inside an office building that requires an RFID key-card to access may have the right to be there (since the company issued them a key card,) yet their behavior within the building may be suspicious. Identifying suspicious behavior in this environment now requires identifying patterns of specific employees-building a database of regular behavior-and then flagging behavior which is out of the norm [9]. While the secretary to the VP of Engineering might have access to the building, it may be suspicious for her to visit the IT Data Center for example; generally we expect IT department employees there and her presence in that location is out of the norm for her at-work behavior.

Defining this norm can come from several ways, but video analysis may be sufficient to identify general traffic patterns when we can't get everyone to wear or carry RFID tags. We can get an idea of what normal behavior looks like for a courtyard or a road simply by watching it for a few hours [33]. Video tracking software can analyze where and how fast people travel through the monitored area. Indeed this is done every day; transportation department traffic cameras on freeway overpasses look down highways and report data to a monitoring station. The purpose is to determine the average speed of traffic [26]. Observers may notice a slow-down during heavy traffic, and an increase in average speed during light traffic. With respect to suspicious behavior: Traffic has a normal direction of flow. Thus, watching somebody drive the wrong way down the highway is certainly suspicious! Recognizing normal behavior for any area is important since it provides reference to start from. 


\section{Examples of suspicious behavior are: [28]}

1. Loud noises and noticeable signals from the agent

2. High speed, since it contributes to surprise

3. Abrupt changes in speed or direction

4. Communication with other agents identified as suspicious

5. Attempting to avoid visibility

6. Isolation and/or lack of communication with others

7. Lack of goal-directed behavior (i.e. loitering and lurking)

\section{Derived Requirements}

Four derived requirements for measuring satisfaction of detecting suspicious behavior are chosen.

This thesis focuses on detecting loitering, meandering, leaving an object and improper location.

Figure 1.2 illustrates the connection between derived requirements and the main requirement.

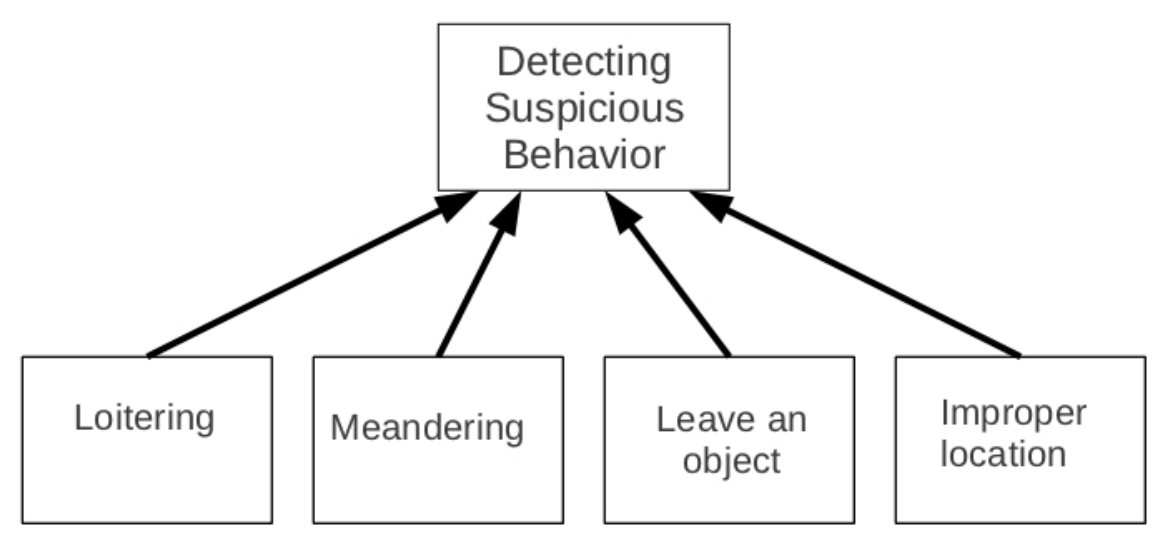

Figure 1.2: derived requirements used in this thesis

\section{Example of behavior: Loitering}

Coordination by deceptive agents may require people to meet in the same location and we may reasonably expect them to spend some time there. While one agent is waiting for another they will 
likely be loitering at the designated meeting place. As explained by Rowe in Detecting Suspicious Behavior from Positional Information, "both an agent that brings a bomb and an agent that installs it must visit the same suspicious low-visibility location" [29]. One real-life example of this is when a Pakistani scientist was found loitering with bomb- making plans in her handbag. It may have been an example of picking the low-hanging fruit since she was caught loitering in front of a police station, however, it became obvious to the police nearby that her loitering was indeed suspicious [39].

\section{Example of behavior: Meandering}

Video tracking was used in a recent paper by Rowe [30] where a test subject was asked to hide around a specific car in a parking lot. The software was able to pick out this individual as suspicious compared with other pedestrians in the parking lot; normal behavior was walking to a car, getting in and driving away, not lurking. Meandering may be important in detecting individuals who are meeting suspicious agents [29] or it may be that someone is acting as a trigger-man for an IED placed nearby.

\section{Example of behavior: Leave an Object}

Obviously, emplacing an IED involves leaving an object, so this is quite an important derived requirement. The U.S. military is testing a system capable of locating ground and sea mines by scanning area with plane-mounted cameras: Joint Multi-Mission Electro-Optic System (JMMES) [40]. JMMES uses: Multi-Spectral Imaging, Mid-Wave Infra Red, color, night, and bioluminescence sensors, integrated and packaged with a Laser Designator/Range Finder in a 15-inch turret. The current status is promising, yet the system needs work. In some tests the system was able to identify disturbed ground where small size land mines were buried, yet it failed in other tests. The system is expensive, requiring high quality optics and an aircraft to position the device.

\section{Example of behavior: Improper Location}

This is the principle behind home alarm systems. When the homeowner has set the alarm and is away, someone else inside the house is clearly in an improper location. The same can be said for being inside a bank vault after operating hours, and the plot of any Mission Impossible episode. A humorous example of detecting improper location appeared recently in a paper by Rowe where video monitoring was used in a quad at the Naval Postgraduate School. This public space contains a landscaped planting area with succulents, bushes and cactus. The expected behavior of subjects 
in the field of camera vision was simply to walk between two buildings. Rowe's software was able to successfully pick out a grad student hiding in the cactus planter in the quad during one test [33].

Other work involves identifying objects and their normal locations within a frame, and then identifying anomalous video images [2]. For example, cars belong on the road and pedestrians belong on the sidewalk. Video of a street showing cars on the sidewalk or pedestrians in the road would be considered an anomalous frame. Cars should not be on the sidewalk, and pedestrians should not be jaywalking. 


\section{Chapter 2}

\section{Related Work}

Four areas of research will be discussed in this section: Low cost magnetic sensors, fiber optic microphones, 3D terrain mapping, and route clearing patrols. The methods described below will each be compared with the three quality attributes of this thesis work:

1. low cost

2. simple

3. proactive vs. reactive

\section{Low cost magnetic sensors}

A few students at NPS have done work in the area of low-cost sensors as detailed below. Both were Master's thesis projects where students studied the Crossbow MSP410 Mote, and specifically the magnetic sensors built into the Motes.

The Master's thesis coauthored by Sundram \& Sim focuses on the Crossbow Motes placed around waste receptacles in public areas. It should be noted that the MSP410 contains a true magnetic sensor; it can detect ferrous materials like a beachcomber's metal detector. Other companies (like Phidgets) manufacture a sensor called a "magnetic sensor" that instead detects magnetic fields. Thus, one can detect a refrigerator magnet but not a box of nails. The experiments carried out in Sundram \& Sim demonstrated that metallic IEDs can be detected by placing the Motes in and around trash cans in public spaces. This is a useful proof of concept, as it is explained that hand-carried explosives 
may be quickly tossed into waste receptacles in markets and parks, causing a great threat to public safety [38].

The Master's Thesis by O'Hara continues the work by Sundram and Sim by experimenting with different placement of the same Crossbow motes to efficiently detect metallic objects [20]. It is more of a sensor placement experiment to determine how far away and what configurations are best. This work creates a testbed of sensors to effectively choose the optimal placement for the magnetic sensor.

The use of the Crossbow MSP410 wireless sensor network works when we examine them in context to our three quality attributes:

1. low cost: The Crossbow Motes use a 2-axis magnetic field sensor which is an inexpensive electronic part, readily available.

2. simple: The MSP410 comes with 1 program loaded onto it, and the units are not meant to be user-serviceable. The academic kits come with a program called Mote-View which is a GUI for Windows OS. It is capable of storing a comma-separated-value file of readings for data analysis with programs such as Matlab. It is simple in terms of being a COTS product, however it is prohibitively difficult to customize the software loaded on the Motes. Normally, Motes are user-programmable, however these are not.

3. proactive: The Motes report in real-time to the sink.

\section{Video Tracking}

Video tracking is the means of identifying objects in the camera's view by comparing the current frame with a reference background image [33]. Location data and speed can be calculated as is frequently done with traffic cameras [26]. Pattern recognition may be used to identify and differentiate vehicles, for example, comparing bright headlights and associating them with a car or motorcycle. Highway cameras judge distance of objects by comparing them with locations on the screen; a camera pointed at the horizon will show objects at the horizon as being farther away than objects at the bottom of the screen. On a highway, the road stripes are painted at regular intervals and are uniform length. Size of vehicle may be calculated by comparing with adjacent road stripes. Generally, highway traffic cameras monitor speed and traffic flow, but they could be used to identify vehicles stopped on the side of the road, or those that have driven to improper locations, (i.e. off the road in 
a ditch.) At time of writing, EarthCam is a commonly available COTS traffic camera. The camera cost is around 1600 USD and is meant to be used with an armored, weather-proof housing which adds another 2000 USD to the bill.

1. low cost: No, cameras and housing units cost several thousand dollars

2. simple: Complex software

3. proactive: Yes

\section{Fiber optic microphones}

Research undertaken by Andrew Lewis for his company SensOptics discovered that traditional dataline fiber optic cables may be used as microphones. The discovery occurred while working on a project for AT\&T to protect buried cables from becoming excavated by nearby construction [10]. The product is currently being sold as a security system for monitoring borders and property lines. Resolution is quite good. Multiple vehicles can be identified along a stretch of $40 \mathrm{~km}$ buried cable, and operators can listen in on specific 10 meter wide stretches of cable [24].

1. low cost: No, system is in the range of $25,000+$ USD

2. simple: No, need to dig a $40 \mathrm{~km}$ long trench. However it does come with user friendly software designed for security personnel.

3. proactive: Yes

\section{D Terrain Mapping}

MetaVR produces software for Unmanned Aerial Vehicles (UAVs) that performs 3D terrain modeling for the purpose of IED detection [19]. These products are based on the principle of using high-mega-pixel CCD cameras and flying over the same area on multiple days. For example, Flight Landata, Inc in Massachusetts uses 60 mega-pixel cameras capable of $4 \mathrm{~cm}$ resolution [37]. Special terrain mapping software can compare data from different days and recognize changes in the terrain, such as disturbed earth or left-objects, (i.e. an animal carcass that wasn't there yesterday.)

The U.S. Navy is testing the Joint Multi-Mission Electro-Optic System (JMMES) [40]. JMMES uses: Multi-Spectral Imaging, Mid-Wave Infra Red, color, night, and bioluminescence sensors, integrated and packaged with a Laser Designator/Range Finder in a 15-inch turret. Testing is still in 
progress; however, it is able to locate buried mines. Another emerging technology is Ground Penetrating Radar(GPR) to locate buried mines [14]. Recent work done at New Mexico Tech in detecting land mines couples GPR with an infrared camera and metal detectors. Generally speaking, terrain analysis techniques involve an entire suite of sensors designed to detect soil changes, whether flown on a UAV or assembled on a ground vehicle.

1. low cost: No, the system requires high-tech cameras mounted in a housing designed for flight, and a UAV

2. simple: No, requires highly complex analysis software

3. proactive: No

\section{Route Clearing Patrols}

Route Clearing Patrols are groups of soldiers using high tech vehicles and equipment (sometimes robotic) to assist in clearing IEDs from a length of road. Vehicles like the Buffalo are armored personnel carriers with a mechanical arm used for exploring and unearthing IEDs buried in garbage or rubble on the roadside. The soldiers themselves undergo extensive training on identifying and locating IEDs [5].

A recent hearing before the 111th Congress illustrates how important human beings are in IED detection:

The Marines frequently patrol with handheld minesweepers, a version of what people use on a beach to find coins. General Mickelson says his best weapons against the bombs is what he calls the mark-one eyeball-that is Marines being over there, soldiers being over there, being there for six months, noticing that strange carcass that wasn't there yesterday is shaped funny with red wire coming out of it, that is the IED [15].

1. low cost: No, human life at risk

2. simple: No, requires extensive training and armored vehicles

3. proactive: No

\section{Overall Results}

Table 2.1 compares the various sensing types mentioned in this section. The most suitable direction of research is the magnetic sensors incorporated into the Crossbow MSP410 Mote. The sensor 
Table 2.1: Overall results comparing related work

\begin{tabular}{|l|c|c|l|l|}
\hline & low cost & simple & proactive & notes \\
\hline magnetic sensors & yes & yes, with reservations & yes & $\begin{array}{l}\text { sensors themselves are } \\
\text { simple, reservations } \\
\text { about user-loadable } \\
\text { programs on MSP410 }\end{array}$ \\
\hline video tracking & no & no & yes & $\begin{array}{l}\text { complicated math in- } \\
\text { volved in program to } \\
\text { compare video }\end{array}$ \\
\hline fiber optic microphones & no & no & yes & \\
\hline 3D terrain mapping & no & no & no & \\
\hline route clearing patrols & no & no & no & \\
\hline
\end{tabular}

electronics are inexpensive, a simple graphing program like Matlab can be used to analyze data, and the wireless sensor network is proactive. That direction is the same as the work we did for our paper in which we focused on detecting suspicious behavior with the infrared motion sensors in the MSP410 [32]. We have reservations about the simplicity of the Crossbow MSP410 Motes because this seems to be the only Crossbow product that does not allow the user to load their own software on the Mote. The user manual describes how only in rare circumstances-in the case of a non-functional Mote-the Crossbow's pre-compiled executable file can be reloaded onto the Mote. It involves disassembling and nearly dismantling the Mote, which is essentially a non-user-servicable product. We had some non-working Motes in the lab and I attempted this only once, however I stopped halfway through and reassembled the Mote because I felt as though I was going to break it. The user was never encouraged to write their own programs for the MSP410. There were things that the Crossbow Motes did that we would have liked to understand better, such as the PIR sensor reporting a subject walking by at their point of closest approach. It would have been helpful to see the factory-implemented algorithm in this case. How did the Motes know when the subject was at closest approach? Sadly, the MSP410 program was not open-source. The Phidgets sensors were highly user-programmable. This is the right direction to go because the user needs to make the system fit their needs and adapt to a specific environment in the field. 


\section{Chapter 3}

\section{Previous Work}

\subsection{Research with IR motion sensors}

Because of researchers like Dr. Rowe we know what direction to move in. Recent research at NPS has contributed to studies on suspicious behavior, studies on likely IED locations, tracking with microphones, using magnetic sensors, infrared sensors and video tracking to detect suspicious behavior.

Work with Dr. Rowe in the Current Direction:

1. Supports automated approach to detecting suspicious behavior with cameras [29]

2. Mathematical model predicts six classic deception strategies [28]

3. Wireless Sensor Network with IR motion sensors tracks detects loitering through linear best fit graph $[32]$

4. algorithms to improve localization accuracy by including spatio-temporal relationships among objects tracked (work leading up to next paper) [27]

5. Predict locations where IED placement will occur, test low-cost COTS sensors (work done for this thesis [34]

\section{Published paper from 2010}

My thesis work builds on that foundation of research on detecting suspicious behavior. Work done in the summer of 2009 was performed using Crossbow MSP410 wireless sensors and published 
in a paper titled: Detecting Suspicious Motion with Nonimaging Sensors [32]. The Crossbow Mote experiments were set up by myself with the assistance of Dr. Neil Rowe, for whom I acted as a Research Assistant. Jose Flores worked with testing microphones and tracking footsteps, under the guidance of Dr. Rowe. The two were put together to illustrate that inexpensive sensors (both binary infrared motion detectors and off-the-shelf Radio Shack microphones) could be used to track motion of pedestrians and detect suspicious behavior.

\section{What was done}

Work during the summer of 2009 involved test cases used to determine the functionality of Crossbow MSP410 Motes with Passive Infrared (PIR) sensors. Detection of suspicious behavior is done by plotting a time-frame of Mote readings and using a statistical best fit program. Skewed data or best-fit-lines that center around one location are an indication of loitering and suspicious behavior. This document presents the basic problem of IED detection and serves as a proof of concept that low-cost sensors may be deployed to monitor roads.

\section{Why use a wireless sensor network?}

The goal is to monitor roads and detect suspicious behavior. Nonsuspicious behavior on a road is continuous travel at a constant speed. A wireless sensor network can be deployed on a road. By its nature, a wireless sensor network is self-repairing; there is a high redundancy among sensors in a well-implemented network, and the ad-hoc routing protocols allow the network to self-repair when a node becomes dysfunctional.

For a description of Wireless Sensor Networks, please see appendix.

\section{Cost is an important factor}

Low cost Motes in a wireless network are preferable to other monitoring devices, such as cameras. Cameras such as those implemented on American highways to monitor traffic are hard to conceal. They have a high cost per unit, (around 3000 for a typical road-use traffic cam) and are subject to problems with occlusion. For example, someone can put up a billboard, and that causes a blind-spot where someone could dig a hole and place an IED unseen by the camera. Other deliberate methods of occlusion are possible, such as shooting out the lens or damaging the mounting hardware. Since 
cameras are meant to monitor a large area (perhaps 50 to 500 meters) the disablement of a camera causes a significant hole in the system.

If the cost of a wireless Mote can get down to 50 dollars (or less) the possibilities of large fault tolerant networks are created. If a Mote can monitor 10 meters diameter, then 50 are needed to monitor 500 meters, at a cost of 2500. This is a savings over the 3000 traffic cam and the usual 2000 dollars for the steel frame mounting tower. If a Mote is destroyed, that in itself is a suspicious act, and Motes nearby can monitor the destructive activity, and alert authorities. 


\subsection{Sensor cost vs. coverage table}

\begin{tabular}{|c|c|c|c|c|c|c|}
\hline Type & $\begin{array}{ll}\text { Cost per } \\
\text { sensor }\end{array}$ & Coverage & $\begin{array}{l}\text { sensors } \\
\text { per } 1 \\
\text { km }\end{array}$ & $\begin{array}{l}\text { Mote } \\
\text { units } \\
\text { per } 1 \\
\text { km }\end{array}$ & $\begin{array}{l}\text { Cost per } \\
\mathrm{km}\end{array}$ & $\begin{array}{l}\text { Temp } \\
\text { Range }\end{array}$ \\
\hline $\begin{array}{ll}\text { XL } & \text { Max } \\
\text { Sonar } & \text { EZ0 } \\
(\mathrm{MB1200})\end{array}$ & $\begin{array}{l}\$ 30 \text { each } \\
\text { or } \$ 120 \text { per } \\
\text { Mote }\end{array}$ & $7.65 \mathrm{~m}$ & 524 & 131 & $\$ 15,720$ & $\begin{array}{l}-40^{\circ} \mathrm{C} \text { to } \\
+65^{\circ} \mathrm{C}\end{array}$ \\
\hline $\begin{array}{l}\text { Bendix VO- } \\
\text { RAD radar }\end{array}$ & $\$ 3000$ & $100 \mathrm{~m}$ & 10 & 10 & $\$ 30,000$ & $\begin{array}{l}-40^{\circ} \mathrm{C} \text { to } \\
+85^{\circ} \mathrm{C}\end{array}$ \\
\hline $\begin{array}{l}\text { KUBE } \\
\text { Cone Optics } \\
\text { TR230 and } \\
\text { C172 Sensor }\end{array}$ & $\begin{array}{l}\$ 10 \quad \text { each } \\
\text { or } \$ 40 \text { per } \\
\text { Mote }\end{array}$ & $5 \mathrm{~m}$ & 800 & 200 & $\$ 8,000$ & $\begin{array}{l}-30^{\circ} \mathrm{C} \text { to } \\
+70^{\circ} \mathrm{C}\end{array}$ \\
\hline EarthCam & $\$ 1600$ & $50 \mathrm{~m}$ & 20 & 20 & $\$ 32,000$ & $\begin{array}{l}-23^{\circ} \mathrm{C} \text { to } \\
+49^{\circ} \mathrm{C}\end{array}$ \\
\hline $\begin{array}{l}\text { Radio } \\
\text { Shack mike } \\
\text { with Icicle } \\
\text { preamp }\end{array}$ & $\$ 87$ & $\begin{array}{l}9.1 \mathrm{~m} \quad(30 \\
\text { feet })\end{array}$ & 110 & 110 & $\$ 9,570$ & $\begin{array}{l}-10^{\circ} \mathrm{C} \text { to } \\
+40^{\circ} \mathrm{C} \\
\text { (guess, } \\
\text { no specs } \\
\text { avail.) }\end{array}$ \\
\hline $\begin{array}{l}\text { Cobalt fiber- } \\
\text { optic micro- } \\
\text { phone }\end{array}$ & $\begin{array}{l}\$ 25,000 \\
\text { (guess) }\end{array}$ & $40 \mathrm{~km}$ & 1 & 1 & $\begin{array}{l}\$ 25,000 \\
+\quad \$ 822 \\
\text { for } 1 \mathrm{~km} \\
\text { of fiber }\end{array}$ & $\begin{array}{l}-40^{\circ} \mathrm{C} \text { to } \\
+50^{\circ} \mathrm{C} \\
\text { for the } \\
\text { cable, } \\
0^{\circ} \mathrm{C} \text { to } \\
+40^{\circ} \mathrm{C} \\
\text { for the } \\
\text { computer }\end{array}$ \\
\hline $\begin{array}{l}\text { Sarasota } \\
625 \mathrm{X} \text { in- } \\
\text { ductive } \\
\text { loop }\end{array}$ & $\$ 137$ & $5 \mathrm{~m}$ & 200 & 200 & $\$ 27,400$ & $\begin{array}{l}-40^{\circ} \mathrm{C} \text { to } \\
+85^{\circ} \mathrm{C}\end{array}$ \\
\hline
\end{tabular}


The above table lists several commercial off the shelf sensors that can be employed to detect suspicious behavior. Hypothetically a wireless sensor network could be built that uses identical Motes, each employing one specific type of sensor. The chart examines how such a network could be set up to monitor $1 \mathrm{~km}$ of road, comparing cost per sensor, range and the number of sensors needed to monitor $1 \mathrm{~km}$ distance. The least expensive setup employs infrared motion sensors: The KUBE Cone Optics TR230 (lens) and C172 sensor. This is basically what Crossbow built when they manufactured the MSP410, and what we employed in our previous work as a proof of concept that a low-cost sensor can be used to detect suspicious behavior [32]. The table shows that while other sensors may be used, the lowest cost solution is the infrared motion sensor.

\section{Microphones were also used to gather data}

Microphone placement can be used to detect suspicious behavior. Nonsuspicious behavior would be a person walking in a single direction at a single speed. Suspicious pedestrian motion is a change in speed (such as stopping) and change in direction. Loitering is an example of both, if the person stops and begins milling about aimlessly. We can measure the differences in time between footsteps to detect a change in speed. Multiple microphones can be used to compute distance of the subject, which in turn can betray a change in direction. Analysis of audio data with a low pass filter is sufficient to detect peaks in sound energy caused by footsteps.

In searching for footsteps in audio data, footsteps are generally not less than 0.48 seconds apart and no more than 0.80 seconds apart, for each walker. Generally a person walks at the same speed, so when we identify a walker we identify nonsuspicious behavior as regular walking with little deviation between peaks, and suspicious behavior as a change in speed, faster or slower.

Similar work in tracking motion through microphones has been implemented by SensOptics, with the Cobalt device. It has been used to monitor cars, as implemented in the fiber optic buried mic [24].

\section{Work done with Crossbow MSP410 IR motes}

To reduce false alarms, we must track changes over a period of time to find consistent patterns [32]. 
The MSP410 Motes contain four passive-infrared (PIR) sensors. They also contain a magnetic change-detection sensor. The magnetic-detection functionality was tested by Sundram in his NPS thesis. Unfortunately, the effective range for detecting a bucket of nails is about 1 yard. Many Motes would have to be deployed in order to cover an entire road, thus the high number required would cause such a system to be cost-impractical.

Work done in Summer 2009 tested the functionality of the PIR sensors. These units are deployed in 4 quadrants around the sensor giving 360 degree view. The sensors were meant to be used as a security system.

\section{Passive Infrared Detection}

The PIR sensors in the Crossbow motes were binary sensors; we found they could only report when a subject was in range but not determine distance accurately. When the Mote reported motion in a quadrant it also reported a PIR strength reading but this was unreliable. We found that when motion was detected this value was 1023, its maximum. Rarely did the Mote report other values.

Accuracy of the PIR sensors decreased exponentially at distance. For example, we were almost $100 \%$ accurate at detecting a person walking past at 12 feet, and almost $0 \%$ accurate at detecting a person walking at 24 feet. This finding was dramatically different than the Crossbow product spec sheet which claimed the MSP410 could accurately detect motion up to 80 feet away. We wondered what battleship they had detected floating by? We noticed that people and cars walking 30 feet away were invisible to the sensor.

\section{Detecting ferro-magnetic metals}

Regularly during testing, a single mote would detect magnetic proximity. The Motes had been placed in a line along the outside of a building, and no metallic objects were immediately visible. At random intervals, one Mote by the corner of the building would give a flurry of magnetic readings. We later discovered that it had been detecting the elevator car moving in an elevator shaft, 2 feet through the wall behind it. 
The MSP410 does have the ability to detect magnetic objects but not at a great range. Previous work (cite thesis) showed that it had a range of about 4 feet. This is helpful for detecting metallic objects such as an IED placed in a trash can in a public market, but not practical for monitoring 40 kilometers of road. An entire roadway would have to be manufactured with cells of magnetic detecting devices. Such a concept may be possible on a paved road of the future, but impractical on a dirt road in Afghanistan. Experimentation showed a fascinating feature of these PIR sensors; they regularly fired off a reading when the subject was at their point of closest approach. It did not seem to matter whether that point was 2 feet away or 12. Tests were performed when the subject's path was linear, U-shaped, and directly toward-then-back. If the subject moved into then out of the PIR sensor's range, the Mote reported a single detection incident. Loitering produced clusters of readings.

(insert picture here)

\section{Proof of Concept that suspicious behavior can be detected with a low cost sensor}

Detecting suspicious behavior was done by analyzing 10 second periods and drawing a best-fit line over the points. A subject moving at a consistent speed in a straight line produced a nice vertical line on our graph in Matlab. When a subject loitered instead of moving through the test space, it produced a diagonal line that fell off the graph on either the left or right side. This rather simplified test illustrated the success of the linear-best-fit graphing technique; namely that clusters of readings produced a skewed line when graphed. Figure 3.1 shows how a single walking subject was tracked with Crossbow MSP410 Motes using PIR sensors.

Previously we stated that the PIR sensors were binary, so location was nearly impossible to determine with accuracy. However, we found that using an estimation was sufficient for detecting suspicious behavior. Since the most reliable detection was from 0 to 12 feet and then reduced dramatically after 12, we chose a point in the center of the possible field, (at 7 feet.) This put the subject in the middle of a detection zone, and was sufficient because a walking person would be in and out of that detection zone in a matter of seconds. It didn't really matter if they approached the sensor at 2 feet distance or 10, because they were moving along to the next Mote in the road 
Linear best fit graph where a subject walks past four PIR sensors (non-suspicious)

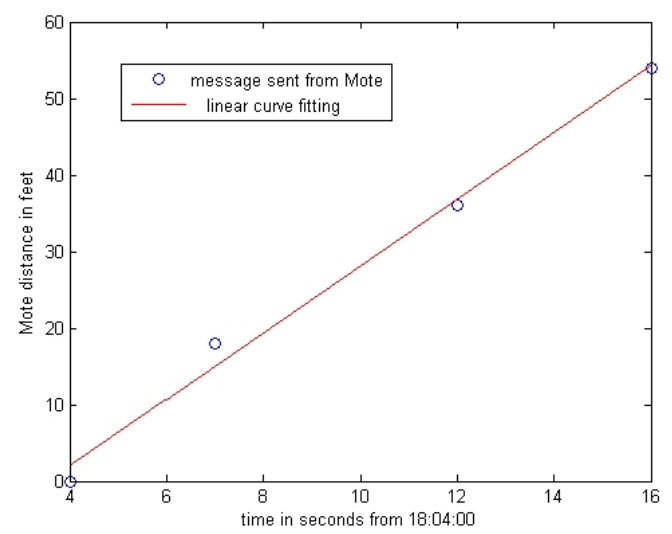

Figure 3.1: Tracking a single subject with Crossbow Motes

anyway. When the subject loitered inside a zone, that produced a flurry of PIR readings. We assume it will take more than just a few seconds to dig a hole to bury a roadside IED. Therefore, stopping is suspicious behavior.

\section{What are we looking for?}

Most IEDs are heavy, weighing in at around 80 to 500 pounds, since they are frequently made from tank artillery shells. Even a coke-can filled with explosives needs to be placed properly. Someone would still have to stop for a few seconds, place the device, and make some reasonable attempt at concealing the IED, perhaps with cardboard, random bricks or concrete rubble. A soda can with a cell-phone taped to the outside would be highly suspicious, and easily detectable. Time must be taken even with small IEDs. It is possible that someone could drop a wrapped package, whether a 12-pack drinks container or a bundle wrapped to look like a submarine sandwich. A smaller bomb of a size that a human being can reasonably carry would do less damage, and perhaps would not damage a vehicle, which most IEDs are intended to do.

In this case, it may be helpful to compare video data over time, to notice when stationary objects suddenly appear in the field [33] [29]. A computer with a camera that tracks people moving through a courtyard for example, would be able to tell the difference between an object that moves into frame and then out of frame a few seconds later [34]. Video tracking algorithms would be able to compare 
earlier data (considered part of the background) with later data to show that a stationary object has been placed in the visual field. This may be the subject of future work to develop a wireless sensor network that detects small, dropped explosives. Since the main goal of our experimentation was to detect people stopping and placing a large IED (which may be heavy and require digging to place) we feel the experiments with the MSP410 were successful. They show that we can detect loitering suspicious behavior, since stopping and digging a hole would certainly fall into that category [32].

\section{Relation to derived requirements}

Because of the nature of IED placement, our experiment will focus on four types of suspicious behavior associated with planting an explosive. Recall that the main requirements our system must meet in order to solve our detection problem are:

- loitering

- meandering

- leave an object

- improper location

We plan to design an experiment to test if other low-cost sensors are capable of detecting these suspicious behaviors. In previous experiments with Crossbow MSP410 motes it was discovered that loitering produced data clustering around one sensor. Thus, we used that as one of our metrics. A linear best fit curve plotted through points showed whether the subject had moved through the area at a constant speed or had slowed down and spent some time in a single area. Simple graphing techniques were sufficient to visually determine if suspicious behavior had taken place. Only basic software was needed to make this judgment; Dr. Rowe wrote the equations and I programmed them into Matlab. Similarly, a good experiment will show how new metrics can be developed and added to the system. It is desirable for non-experts in the field to be able to adapt the system using simple software. College-level math was all it took to see that a linear-best-fit line was suitable for detecting loitering in our 2009 work. When new metrics are developed, a new equation, algorithm or program can be developed and a new component can be added to a system designed to detect specific types of suspicious behavior. 


\section{Chapter 4}

\section{Experiment Design}

\subsection{Black-box testing of sensors (see appendix A for experi- ment date \& charts)}

\section{Metric Development}

Data patterns emerge in experiments where suspicious behavior takes place, which gives us the ability to develop Metrics, as seen in Figure 4.1:

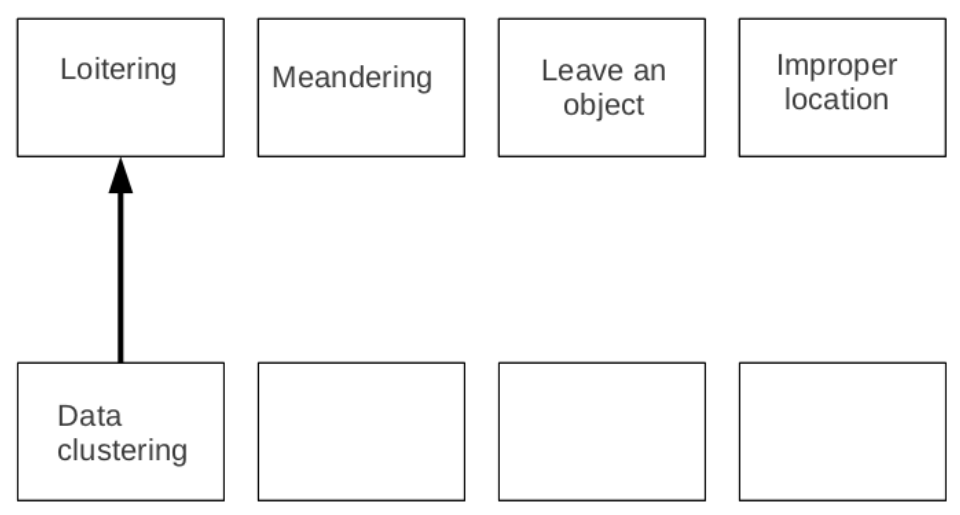

Figure 4.1: Developing metrics for derived requirements

The chart above looks like it did before we started experimentation; there are no lines linking data patterns to specific types of suspicious behavior, except in the case of data clustering being 
related to loitering. The other data patterns need to be identified by doing experiments. We need to examine what datapoints appear over a given threshold, for a given sensor, when we perform a specific activity in a controlled experiment. For example, during our experiment in the Glasgow Hall Quad, we placed objects, went to strange places on the map, and we also meandered without walking directly through the quad. These allow us to record sensor readings during activities we have defined as suspicious, so that we can analyze that data and see what patterns emerge. In keeping with the design concept of simplicity, we analyzed the over-threshold data in a COTS software suite called Matlab. Simply looking at a graph can help us identify what is suspicious.

For example, in the experiment below, (see Figure 4.2) a human subject walked through the quad and came back. This is a normal activity, namely walking through the quad as if we are going somewhere. This corresponds to someone walking from the bottom of the field to the top, and back again:

In the next example, (see Figure 4.3) the human subject meandered around the left side of the Quad (locations 5-6-7) for 60 seconds, then moved on. There is a definite pattern in the graph where the subject meandered around between locations.

Such behavior (on the graph it looks like a zig-zag) is definitely abnormal. It indicates that someone meandered around a certain area for a instead of walking straight through. It was by graphing that we found data patterns that happened, and were unique when suspicious activity occurred. The entire experiments and graphs are shown in the appendix for the Glasgow Quad Experiments, and one may see how we determined the metrics from graphed data patterns there.

Only after experimentation were we able to manufacture metrics for detecting suspicious behavior; patterns emerged as the data was being analyzed in Matlab. In fact, Matlab was a very helpful tool because the data could be sorted with different threshold values and then graphed to determine if patterns emerged. Not only did this validate the thresholds we had chosen but it also let patterns emerge that were clear examples of the metrics we were trying to find. Therefore, through experimentation the following patterns emerged: (see below and Figure 4.4.) 

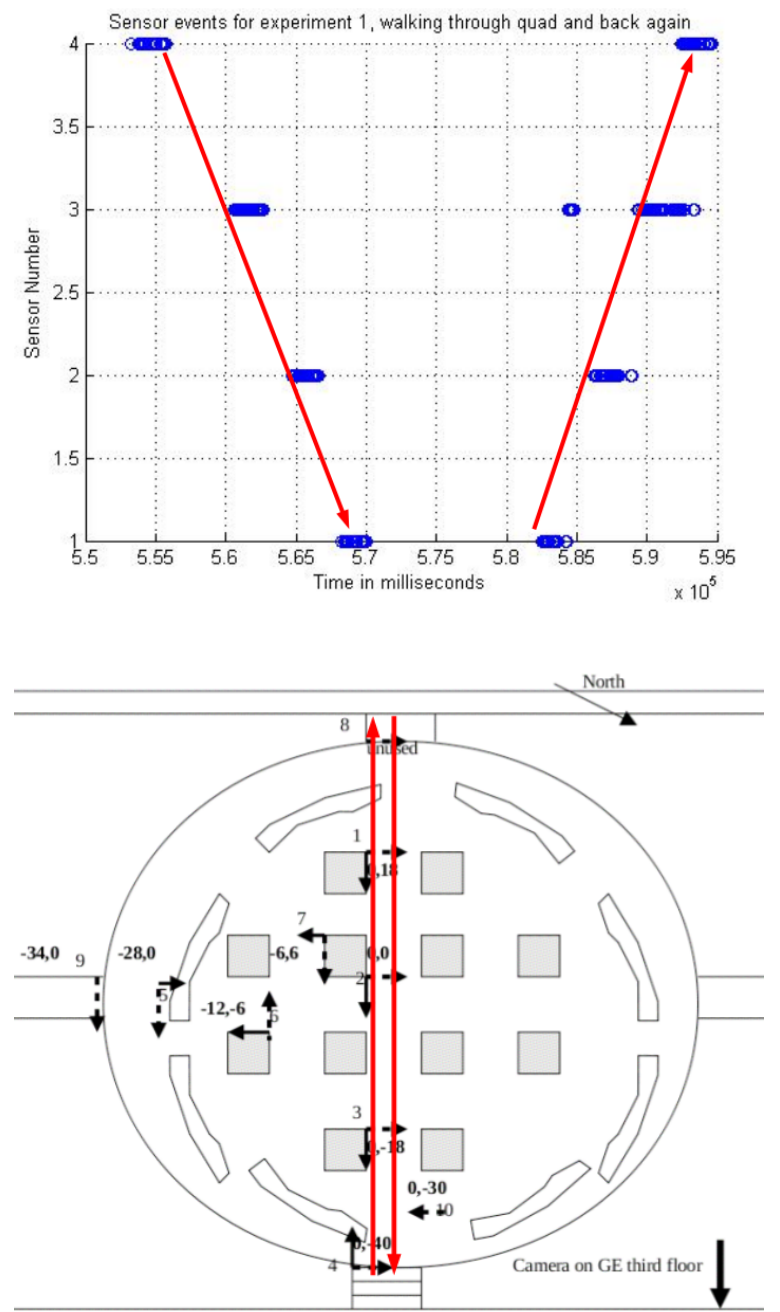

Figure 4.2: Normal activity measured in Glasgow Quad 

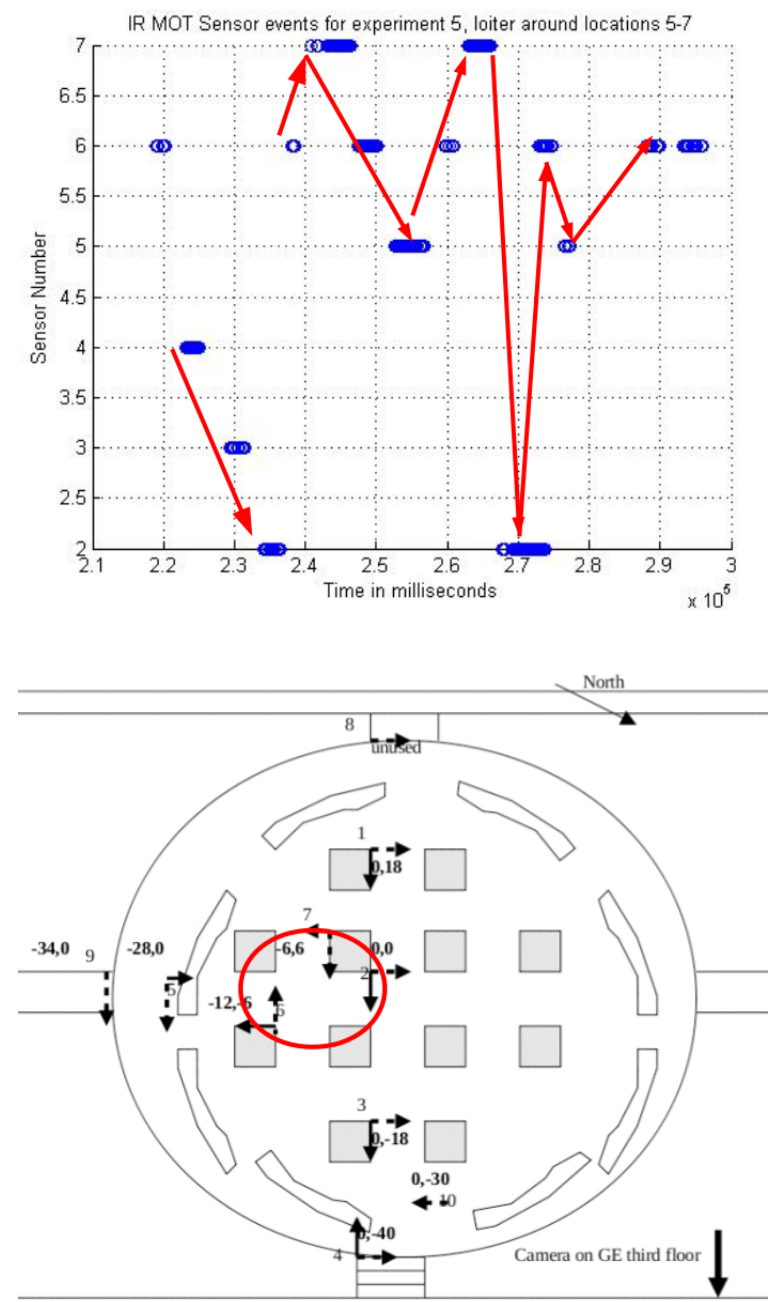

Figure 4.3: Suspicious activity (loitering) measured in Glasgow Quad 


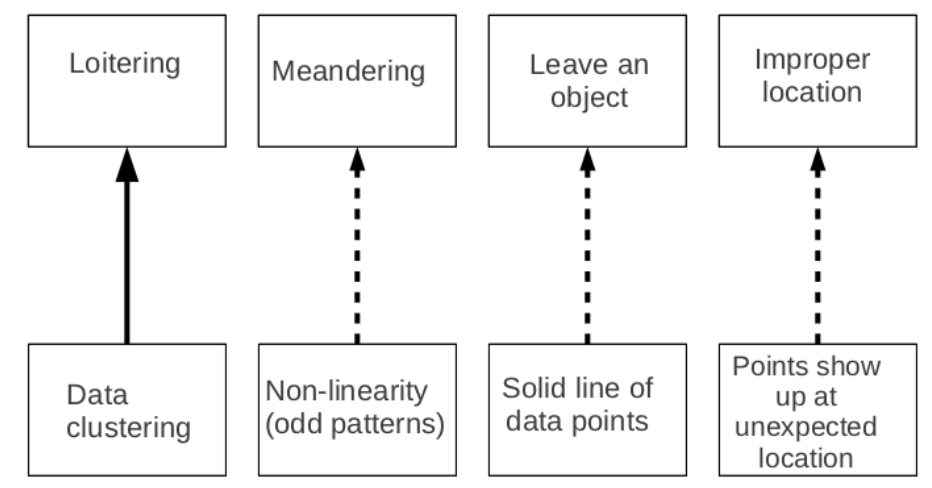

Figure 4.4: Linking metrics to derived requirements

\section{Development of Metrics after experimentation:}

1. Data clustering was associated with Loitering

2. Non-linearity (odd patterns) associated with Meandering

3. A solid line of points corresponds to Leaving an Object

4. Significant points appearing at unexpected locations indicates Improper Location

\section{Overall Experiment Design}

Designing the Experiment: We set out with the need to design experiments to test various sensors against our requirements and attributes. Experiments are designed in a way such that we can analyze the data with simple methods, and develop the correct metrics.

In a nutshell:

1. choose some simple sensors

2. design simple software

3. prototype and test to see how well we can do

Developing our testbed: Work done by Dr. Rowe shows likely places for IED emplacement, as seen in the diagrams below [34]. This guides our experiment setup and testbed development. Video 


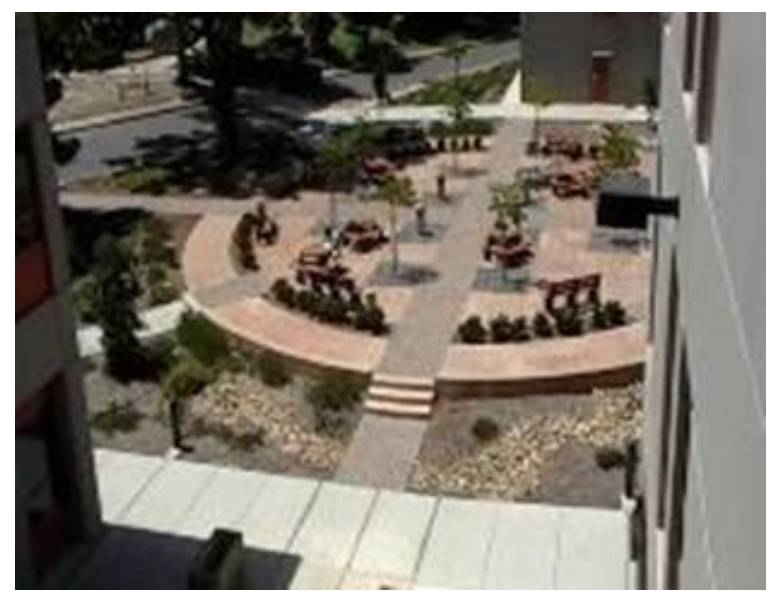

Figure 4.5: Glasgow Hall Quad image courtesy N. Rowe

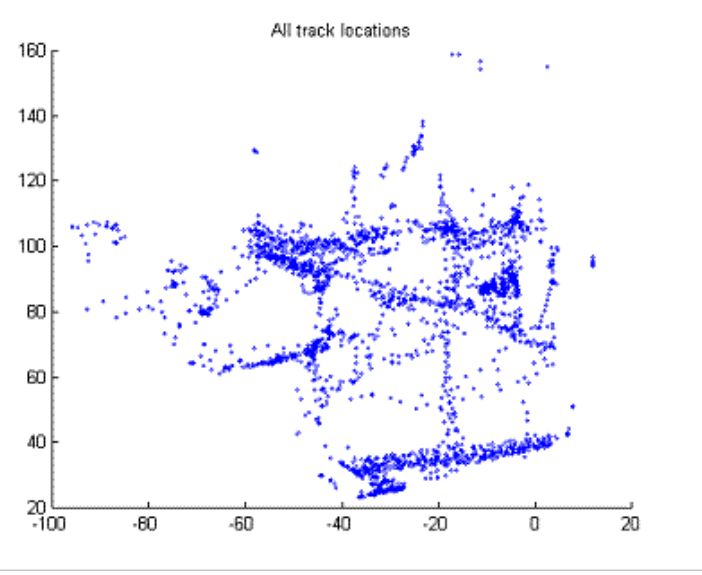

Figure 4.6: Tracked paths through Glasgow Hall Quad image courtesy N. Rowe

analysis was done in the Glasgow Hall quad area at NPS. Figure 4.5 shows a still shot from this video analysis. Paths were plotted using Matlab software, as seen in Figure 4.6. Based on frequently traveled paths, likely locations for IED placement were determined; the larger circles in the diagram indicate a high probability of IED placement. This can be seen in Figure 4.7. Our prototype-sensor network was set up according to this analysis.

\subsubsection{Preliminary Experiments}

Our first question: Out of list of commercially available (COTS) hardware sensors, Which ones are feasible to use in a testbed? 


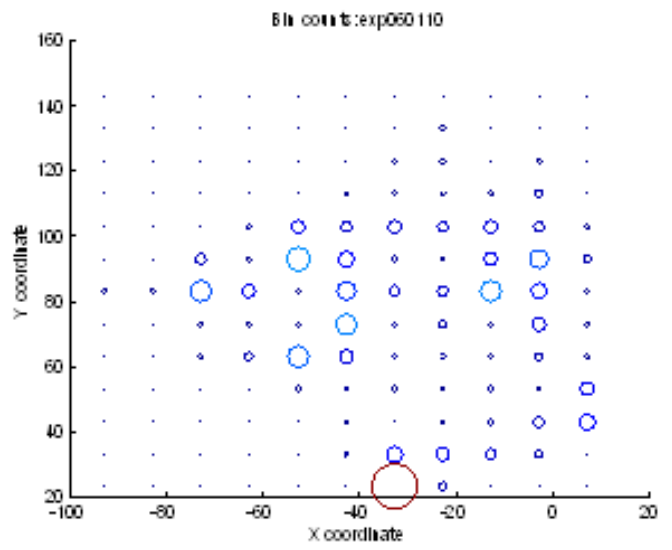

Figure 4.7: Likely IED placement in Glasgow Hall Quad image courtesy N. Rowe

XL Max Sonar -EZ0 (MB1200)

Phidgets 1120 pressure strip

Bendix VORAD radar Phidgets 1110 touch sensor

KUBE Cone Optics TR230 and C172 Sensor

Phidgets 1104 vibration sensor

EarthCam - highway traffic camera

Phidgets 1111 infrared motion sensor

Crossbow MSP410 (8 per kit)

Phidgets 1127 Precision light sensor

Radio Shack mike with Icicle preamp

Phidgets 1128 sonar distance sensor

Cobalt fiber-optic microphone

Phidgets 1101 \& 3521 IR distance

Sarasota 625X inductive loop

Phidgets 1108 magnetic sensor

Phidgets 1106 force sensor

The decision about what to use was based on cost.

\section{Eliminated:}

XL Max Sonar -EZ0 (MB1200), used in Phidgets $\$ 30$

Bendix VORAD radar $\quad \$ 3000$

KUBE Cone Optics TR230 and C172 Sensor, (used in Phidgets IR motion) $\$ 10$

$\begin{array}{ll}\text { Crossbow MSP410 educat. kit (8 per kit) } & \$ 4000\end{array}$

$\begin{array}{ll}\text { EarthCam - highway traffic camera } & \$ 1600\end{array}$

$\begin{array}{ll}\text { Sarasota } 625 \mathrm{X} \text { inductive loop } & \$ 137\end{array}$

Cobalt (OptiSense) fiber-optic microphone $\$ 25000$ (est) 


\section{Purchased:}

Radio Shack mike with Icicle preamp $\$ 87$

Phidgets 1120 pressure strip $\quad \$ 23.45$

Phidgets 1110 touch sensor $\quad \$ 7.25$

Phidgets 1104 vibration sensor $\quad \$ 11.35$

Phidgets 1111 infrared motion sensor $\$ 46.35$

Phidgets 1127 Precision light sensor $\quad \$ 11.35$

Phidgets 1128 sonar distance sensor $\quad \$ 36.05$

Phidgets $1101 \& 3521$ IR distance $\quad \$ 11.35+\$ 12.40$

Phidgets 1108 magnetic sensor $\quad \$ 11.35$

Phidgets 1106 force sensor $\quad \$ 11.35$

\section{The need for black box testing}

Reverse engineering was necessary to determine the specifications for each of the chosen sensors. Product specification sheets are frequently works of fiction, as we found out with the Crossbow MSP410 Mote in 2009 [32].

Passive infrared (PIR) sensors are used to detect dynamic changes in the local thermal radiation environment. The MSP410CA is equipped with four separate PIR sensors arranged orthogonally, providing full 360-degree coverage. This Quad Detect capability enables the identification of initial object vector as well as subsequent movement and direction. These high performance sensors can detect both personnel and vehicles at distances of up to 80 feet or more [16].

First, the spec sheet implies that a binary sensor can identify object vector. We did not find that was the case; the IR Motion sensor simply relates whether or not it sees an object within its range. Determining a vector has to be done with plotting and tracking between multiple sensors. Secondly the PIR sensor has a probability that it will detect someone, thus it does not have $100 \%$ accuracy, nor can it see to 80 feet. It only had a $53 \%$ chance of seeing someone at 30 feet. 


\section{PIR probability distribution of detecting a walking person}

$\begin{array}{llllll}30 \text { feet } & 27 \text { feet } & 24 \text { feet } & 21 \text { feet } & 18 \text { feet } & 15 \text { feet } \\ 0.53125 & 0.65625 & 0.84375 & 0.90625 & 0.96875 & 1.0\end{array}$

\section{Hypothetical uses of sensors}

Of the Phidgets sensors chosen, we had initial hopes they would perform in ways that would detect suspicious behavior. Here were our initial thoughts:

\begin{tabular}{|l|l|}
\hline Sensor & Hypothesis \\
\hline Phidgets 1120 pressure strip & Detect exact location of subject \\
\hline Phidgets 1110 touch sensor & Detect exact location of subject \\
\hline Phidgets 1104 vibration sensor & Detect footsteps \& infer distance \\
\hline Phidgets 1111 infrared motion sensor & Detect subject in vicinity \\
\hline Phidgets 1127 Precision light sensor & Unsure if we could detect shadow of passing subject? \\
\hline Phidgets 1128 sonar distance sensor & Better than IR Motion, these are more than binary \\
\hline Phidgets 1101 \& 3521 IR distance & Same as above \\
\hline Phidgets 1108 magnetic sensor & Detect artillery rounds \& explosives with metal casings \\
\hline Phidgets 1106 force sensor & Detect object placement \\
\hline
\end{tabular}

The observed behavior of sensors follows in the next section. Reverse engineering allowed the sensors to be tested to see if they should be used in the next phase of the experiment, namely the setup of prototype wireless sensors.

\subsubsection{Reverse engineering sensors to determine specifications}

Phidgets manufactures a large array of sensors and we ordered over a dozen different models. These sensors had to pass an Initial Stage before they got to the more thorough black-box investigation. Initial testing was very basic: The Phidgets sensors were set up on the desktop and we played around with them. Some of the Phidgets sensors we purchased did not even pass muster, such as the mechanical slider which reports it's position (i.e. 50\%.) Sensors deemed unsuitable were thrown back in the drawer and never made it to our eventual list of nine sensors to Black Box test.

Black box testing is important because the vendor's product information sheets can sometimes be works of fiction. Also, sometimes our own hypothesis about how they will perform is not accurate. 
One example is the magnetic sensor; we thought it would behave like a metal detector, and instead it only detected magnets. It did not detect ferrous objects like we anticipated. A value judgment was made simply on the basis of whether or not a sensor should be used in the next phase: Pass or Fail.

The following Phidgets Sensors devices were thoroughly black-box tested:

1. physical contact sensors

(a) 1120 pressure strip

(b) 1110 touch sensor

(c) 1106 force sensor

(d) 1104 vibration sensor

2. 1111 infrared motion sensor

3. 1127 precision light sensor

4. 1128 sonar distance sensor

5. 1101 distance adapter and 3521 IR distance sensor

6. 1108 magnetic sensor 


\subsubsection{Physical Contact Sensors}

\section{Pressure Strip (1120 FlexiForce Adapter and thin-film Sensor)}

\section{Black-box testing: successful}

This is two devices: A Phidgets adapter and a thin film sensor strip. The strip is four feet long and one inch wide. Combined, this is a good sensor to detect human subjects. We placed the thin film sensor under a 2 foot square of carpet to avoid damaging the strip. When placed underneath a carpet square it is able to detect when people step onto the carpet. This sensor will send interrupts to the Phidget Interface Kit when change occurs over a certain threshold. In experiments, we placed a laptop computer over the carpet square (with sensor underneath). It reported 1 interrupt upon pickup, 4 interrupts upon placing the laptop back on the carpet.

The pressure strip should be used as a binary sensor, not a weight scale. It is able to take the weight of a human being with no trouble, as long as the pressure strip remains flat and is not bent or deformed in any way. The product spec. says it is meant for objects 0-100 pounds but we had no problem stepping on it, when placed under a carpet square. However the device is delicate and should not be used in an environment like a dirt road in Afghanistan. Also, people can easily step over the sensors or go around them.

In the experiments where we placed a laptop on the carpet square concealing the pressure strip, we got consistently different readings. This was not because the weight of the laptop changed, or the pressure strip was faulty. We realized that sometimes the rubber feet on the laptop were pressing directly on the pressure strip and other times the laptop feet were bridging the pressure strip. In these cases it was only detecting pressure on the carpet square itself. Placing a laptop on the carpet square with the pressure strip caused it to report a raw value of around 450 .

Due to the structure-and delicacy-of the plastic sensor strip we believe this sensor is only suitable for hard, flat surfaces. Placing it under a carpet square on concrete and then walking over it is acceptable. Placing the sensor under dirt or on top of gravel would probably damage it.

The sensor itself is a Tekscan Flexiforce sensor. This is a paper-thin printed circuit. The thin-film 
construction allows for flexibility however care should be taken with the sensor so it does not break.

\section{Touch sensor}

\section{Black-box testing: fail}

The touch sensor is not good for detecting the placement of an object. The touch sensor will tell you if you are touching it. It's pretty basic. Think of it as a doorbell, rather than a weight scale. The product specification says: "The 1110 is a capacitive touch sensor and can detect a touch through plastic, glass, or paper. We recommend a material thickness of $1 / 8$ inch."

\section{Force sensor}

\section{Black-box testing: successful}

The force sensor is simply a push button covered in rubber; it will tell you how hard something is pressing the button. The button has a physical limitation of $1 \mathrm{~kg}$, so please do not push hard on the button or roll a bowling ball over it. You will break it. The Phidgets web site says, "Intended as a user input device (i.e. recognizing that someone is pushing a button.) Not accurate enough to be used as a weight measurement device."

The Force Sensor returns a Raw Value to the Phidget Interface Kit. Low values indicate a little force and high values indicate a lot of force, up to its maximum reporting range. This sensor will report raw values between 0 and 4000. In a simple test, an 18 inch by 18 inch carpet square with a heavy rubber base was placed on top of the Force Sensor and made it report 2642. Placing a laptop

computer on top of the carpet square make the Force Sensor report 3944, its maximum value. You wouldn't want to put anything heavier on top of the sensor.

\section{Vibration Sensor}

\section{Black-box testing: questionable}

This sensor is an inexpensive Piezo-electric disc which reports a change when a thin film flexes. We tested the device four different ways, 1. taped to the ground (using electrical tape), 2. resting on the ground, 3. mounted to a block of wood, 4. mounted inside an Altoids tin. We hypothesized that the senor could detect footsteps and human subjects walking past. This would be useful for 
tracking subjects and detecting suspicious behavior.

When the sensor is taped to the ground, it is not able to detect footsteps nearby. It simply reports whenever the wind blows over the sensor (flexing the thin film.) Because it is taped to the ground, the Raw Data Values reported are subdued.

When the sensor is resting freely on the ground it reports wild readings every time air flows over the device. The sensor is free to flex and reports whenever the wind blows. It fails to report footsteps nearby. Thus, this is a physical contact sensor because it can only detect when the object it is mounted to is actually moving. It may be suitable to detect when a bridge is vibrating when mounted directly to a bridge.

\subsubsection{Infrared Motion Sensor}

Black-box testing: successful This sensor is manufactured by Panasonic, part number AMN23111. The Panasonic data sheet discusses the detection zone and says, "the detection zone patterns are indicative of the projections of the 20 lenses with single focal point and with five optical axes. An object whose temperature differs from the background temperature and which crosses inside the detection zone will be detected."

This sensor does adjacent-cell-comparison to detect motion, similar to the human eye. Multiple fields monitored in the sensor and comparisons are make between adjacent fields. Experiments have shown the sensor is better at detecting side-to-side motion and worse at detecting motion coming straight towards it.

Characteristics for this Phidgets sensor as per the 1111 Product Manual:

This sensor detects changes in infrared radiation which occur when there is movement by a person (or object) which is different in temperature from the surroundings. As this sensor detects temperature differences, it is well suited to detecting the motion of people by their body temperature. The sensor is also characterized by a narrow sensing area. 
The product spec sheet says it can detect a human being at 5 meters away, with an operating temperature of $-20^{\circ} \mathrm{C}$ to $+85^{\circ} \mathrm{C}$.

Experiments show that at rest, the raw values are high, around 2000. When the sensors pick up motion the raw values drop to some numbers less than 100. The lowest readings (indicating the most motion) are around 99. Also, the sensor can go high and give a reading around 3000. This also indicates a detection event. Therefore, a threshold of $+/-1000$ from the "at rest" measurement of 2000 is a safe bet.

Analysis: A threshold value must be selected when polling the sensors. This may be between 500 and 1000 depending on what accuracy the user chooses. The higher the threshold the closer an object must be in order to trigger a detection event. The Phidget Interface Kit can be set up to detect interrupts from the sensors, however this must also be used in conjunction with a userprogrammable threshold. Otherwise the device may report many false positives if a low threshold is used.

Ranges: Tests were performed with the sensor laying on the ground, (cone pointing up) and walking nearby. When a human subject walks directly toward and then away from the sensor, the range is only 1 yard. When someone is walking in a circle around the sensor, it has an accurate range of 3 yards. Detection further out depends on the threshold you set.

The device can detect someone at 4 yards if the threshold is set at 500. If the threshold is set at 1000 (meaning the Raw Values must change by over 1000 points) there is no detection of subjects at this range. The device is not able to detect someone who is standing still. The device operates by comparing several areas of its visual field and responding to changes between areas. If someone stands still, there is no visual change detected. In contrast, the Infrared Distance Sensors are able to detect someone standing still.

\subsubsection{Light Sensor 1127}

\section{Black-box testing: successful}


The Phidgets 1127 Precision Light Sensor is meant for indoor use. This sensor measures light level in lux from 1 lux to 1000 lux, in a narrow range. The peak sensing wavelength is $580 \mathrm{~nm}$. This is at the end of the color yellow, moving towards green. The sensor works very well indoors and can detect subtle changes in brightness around different areas of a room. It is able to work under industrial fluorescent bulbs.

The typical bulbs installed in classrooms at NPS are Phillips Alto Universal T8 bulbs. These are long-life (20,000 hr) low mercury tubes intended for industrial use. Bulbs are 32 Watts. Design lumens for the bulbs are 1200 to 2800 . The school is using a mix of bulbs with color temperature ratings of $3500 \mathrm{k}$ and $4100 \mathrm{k}$. Fluorescent bulbs generally have two spikes in color because of Mercury; one energy spike appears in the violet $(435 \mathrm{~nm})$ and another in the green (546nm.) The Phidget Precision Light Sensor has a Peak Sensitivity Wavelength @ 25C of 580nm. We believe the sensor to be a Panasonic AMS104, which has at least 0.7 relative sensitivity at $546 \mathrm{~nm}$ according to the spec sheet chart.

This sensor can not function in daylight outside. It requires several layers of filter material in order to bring the light exposure down to a range it can measure, otherwise the readings are peaked to maximum. We find that using 5 layers of smoked glass with $33 \%$ filtering will put the sensor in a functional range outdoors. With this kind of filter stack it can be used to detect the shadow of a passing object. Light transmission with 5 filters at $33 \%$ is $0.391 \%$. $(.33 \times .33 \times .33 \times .33 \times .33)$. Most of these sensors are designed for home robotics kits, so it makes sense that they would function better indoors.

We experimented with placing the sensors inside cardboard boxes and creating a window in the side. This window was covered with 5 layers of limousine-tint film (90\% blockage).

Sensors report a Raw Value of 0 to 4000. To find the value in Lux, the following formula is used:

Lux $=$ RawSensorValue/4.095

When this is used, a peak value of 950 is considered saturated. Otherwise a raw value over 3800 is considered too bright. 


\subsubsection{Sonar Distance Sensor 1128}

\section{Black-box testing: successful}

This Phidgets sensor incorporates a MaxBotix EZ-1 Sonar Sensor, which can be seen in Figure 4.8. The sensor is very noisy; it produces a lot of spikes which could be considered false-positives of an object in view. Differentiating between suspicious behavior and normal behavior becomes more difficult when the data contains noise. As stated in Chandola et al.

Often the data contains noise that tends to be similar to the actual anomalies and hence is difficult to distinguish and remove [7].

Say for example a sensor reports values in the range of 2000 when nothing is in view (as is the case with this sensor.) Data is being sampled at 10 times per second, and may look like this: [2000, 2000, 999, 2000, 2000...] If a researcher chooses a specific threshold, say 1000, and writes a program to pull out tuples of raw data from the sensor that fall below that threshold, 999 may be seen as a valid data point. However, we know that a human subject didn't just jump in front of the sensor for one-tenth of a second and move away, since we are monitoring pedestrians on a sidewalk. Some kind of noise filtering should be used when polling data. A journal article was found that contained a filter that was easily incorporated into Java code for the Phidgets:

A Recursive Exponential Filter For Time-Sensitive Data, LAUR-99-5573, Phillip D.

Stroud, Los Alamos National Laboratory, October 8, 1999 [36]
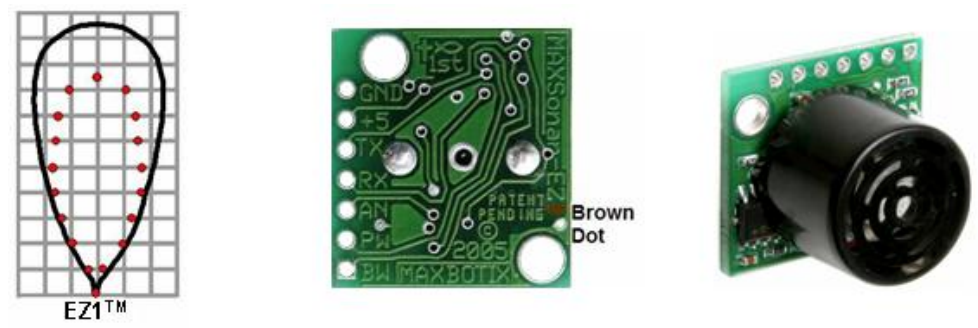

Figure 4.8: The MaxBotix EX-1 sonar sensor field of vision

This shape was verified during angle testing:

- Sonar can see 5 yards at 0 degrees

- Sonar can see 5 yards at 10 degrees

- Sonar can see 2 yards at 20 degrees 
- Sonar can see 1 yard at 30 degrees

- Sonar can not detect a subject at 40 degrees.

The sonar sensor reports Raw Values in the range of 2020 when it sees to infinity and there are no objects in view. This number becomes lower when objects move closer. The Phidgets specification sheet states this sensor is capable of sensing objects up to 6.45 meters away, however this claim was not observed during testing. We found a maximum range of 5 yards, (4.57 meters.)

The Phidgets 1128 specs give a conversion equation for dealing with Raw Values. The Formula to translate SensorValue into Distance is:

$$
\text { Distance }(\mathrm{cm})=\text { SensorValue } * 1.296
$$

Where SensorValue is determined by:

$$
\text { SensorValue }=(\text { RawSensorValue/4.095 })
$$

The accuracy was found to be off. Errors at distance were:

$$
\begin{array}{ll}
1 \text { yard } & 0.21 \text { yard error } \\
2 \text { yards } & 0.51 \text { yard error } \\
3 \text { yards } & 0.64 \text { yard error } \\
4 \text { yards } & 0.98 \text { yard error } \\
5 \text { yards } & 1.13 \text { yard error }
\end{array}
$$

The errors were all such that the object registered as being closer to the sensor than it really was. For example, at 4 yards, (12 feet) the sensor would report that a person standing still was 9.05 feet away. At five yards (15 feet) the sensor reported that the person was standing 11.66 feet away. When a human subject stood 6 yards away the sensor reported values above 2000, the same as when nothing is in view. This error is helpful in tracking a subject, but it is not necessary for detecting suspicious behavior. If someone is loitering in the area, we can rely on the sensor as a binary sensor; we simply care whether or not someone is standing in the immediate area. Standing in that area may be suspicious all by itself. 


\subsubsection{Phidgets 1101 IR Distance Adapter and 3521 Infrared Distance Sensor}

\section{Black-box testing: successful}

This sensor is two devices: the 3521 Sharp Distance Sensor and the 1101 IR Distance Adapter. The Sharp IR distance sensor is meant to be used with home robotics kits. It is supposed to be accurate from $10-80 \mathrm{~cm}$. When objects are closer than $10 \mathrm{~cm}$ it just reports values close to the 10 $\mathrm{cm}$ range. When nothing is in sight it reports a raw value around 18-20. When an object is very close it reports values 800-900.

The 1101 Phidgets manual gives the following formulas:

The formula to translate SensorValue into Distance for Sharp 10-80cm analog sensors is:

Distance $(\mathrm{cm})=4800 /($ SensorValue -20$)$

This formula is only valid over the SensorValue range 80-500.

To adjust a formula, substitute (SensorValue) with (RawSensorValue / 4.095)

Experiments found the IR Distance sensor could be used like a binary beam-sensor. This operates similarly to an "electric eye" beam crossing a door in a retail shop. When a customer walks through the door the beam is broken and a bell rings. The Sharp IR sensor can be used as a binary sensor in this fashion: It has no accuracy for distance measurement, yet it can tell if an object is within range out to 3 yards. The field of vision is pencil thin.

IR distance sensor can see 1 yard at 10 degrees $\backslash 1$

IR distance sensor can see 3 yards at 0 degrees $\backslash 1$

Thus, it has a range out to 3 yards. During experimentation it reported raw values in the $300-500$ range for objects crossing at the 1 and 2 yard marks. It reported a raw value of 109 when an object was 3 yards away, and 23 when an object was 4 yards away. It reports a value of 18-20 when nothing is in sight, so we concluded that it didn't detect anything at 4 yards.

Using a range of 3 yards, we suggest a threshold of 100 points. In the example above, the sensor 
reported a raw value of 20 when nothing was in sight, and a raw value of 109 when a subject was standing at 3 yards distance; thus we see a change of 89 points. Setting a threshold of 100 assures us with a high degree that someone is within the 3 yard range.

\subsubsection{Phidgets 1108 Magnetic Sensor}

\section{Black-box testing: fail}

This sensor was practically useless in testing. We had previously encountered a "magnetic sensor" in the Crossbow MSP410 Motes, and in fact, somebody at NPS wrote their whole Master's thesis on how they can be used to detect IEDs thrown in garbage cans [38]. The MSP410 has a, "two-axis magnetic field disorder detector." Sundram performed experiments carrying a bucket of nails past the Mote to see how far away it could detect ferrous objects. It should be noted that the nails were the typical hardware-store variety. They are simply steel nails, not magnetic by themselves. Going on the success of Sundram's thesis, we chose to purchase the Phidgets Magnetic Sensors, believing they would behave the same way.

Experiments showed they were not capable of detecting a bucket of nails at all. Instead, as we later found out, they are magnetic-field detectors. They don't detect ferro-magnetic objects (like nails) they detect magnets themselves, or in other words, they detect magnetized material. The only way we could get the Phidgets sensor to do anything was to drop a magnet directly on the sensor. In a nutshell, the Crossbow sensor will detect your refrigerator, the Phidgets sensor will detect your fridge magnets.

The Phidgets sensor may have more application in a home robotics kit, to determine when a toy train has hooked up to another toy train car. They're not great for detecting terrorists. 


\section{Summary of Test Results}

Overall results of black-box tests:

\begin{tabular}{|r|r|r|}
\hline Sensor & Black Box Testing & reason \\
\hline 1120 pressure strip & successful & precise location \\
\hline 1110 touch sensor & fail & must be touched on purpose \\
\hline 1106 force sensor & successful & detected object placed \& removed \\
\hline 1104 vibration sensor & questionable & won't detect motion nearby \\
\hline 1111 infrared motion sensor & successful & useful in tracking \\
\hline 1127 precision light sensor & successful & can detect shadows \\
\hline 1128 sonar distance sensor & successful & works if subject stands still \\
\hline $1101 \& 3521$ IR distance & successful & useful in tracking \\
\hline 1108 magnetic sensor & fail & doesn't detect iron \\
\hline
\end{tabular}

Of the sensors mentioned as successful above, the infrared motion sensor, light sensor, sonar sensor and IR distance sensor are our favorites for detecting suspicious behavior. The pressure strip is useful in exercises and should be included in experiments, but it is delicate and should not be deployed in the field. For example, a dirt road in Afghanistan is certainly a lot different than the brick paths at a naval base. As well, the force sensor is useful in experimentation to tell exactly when a device has been placed or removed. The sensor itself is delicate and would easily be damaged in the field. The concept of a pressure sensor and a force sensor are useful, and further advances in technology may show that a durable, field-deployable version exists down the road. 


\subsection{Prototype testing in field (see appendix B for experiment data \& charts)}

\subsubsection{Experiment in Quad, proof of concept}

Note: Full graphs of each sensor's performance in each of 10 experiments appears in Appendix D. There are approximately 40 graphs there (too much information to place here in this section.)

Four experiments were set up in the quad in front of Glasgow Hall at the U.S. Naval Postgraduate School in Monterey, California. Each experiment used around ten laptop computers connected to various sensing equipment (infrared motion detectors, light sensors, microphones, etc.) The laptops were placed in the quad along two main paths (one path north-south, another west-east.) Ten experiments were scripted where one or more individuals would walk through the quad on various paths. Participants were scripted to behave normally during some experiments, and behave suspiciously in other experiments.

Examples of suspicious behavior tested were:

- Stopping along the path and placing a canvas bag with heavy objects inside (books).

- Stopping along the path to pick up the canvas bag.

- Walking to the middle of the quad and loitering

- Digging in the landscaping behind a bench (simulated IED placement)

- Dragging a shovel through the courtyard

\section{Build-up to the final experiment}

The reason we did four experiments was because we either experienced set-up problems, or we decided to rearrange the placement of the laptops in the field. For example, the wind in the summer in Monterey is pretty good; we had days of steady $12 \mathrm{mph}$ wind (NPS is located 1 block from the ocean). The Phidgets sensors all connect to a PhidgetInterfaceKit with thin red-white-black wire twists. They tended to move slightly in the constant wind, causing interference with data collection. The Phidgets motion sensors detected their own wires, for example. Thus in future experiments we taped them down quite well to eliminate this error. 


\section{Developing a prototype}

Each laptop in the field represented a prototype sensor. When a full-featured product is developed based on this work, it is intended that each unit is a wireless sensor node in an ad-hoc network. Please see the future work section for more detail. The proof of concept is meant to test possible sensor configurations. Hence, we have configurations with microphones, IR motion sensors, sonar distance and IR distance sensors. Four laptops had light sensors attached, and one had a pressure (force sensor) attached for the purpose of testing. The Phidgets force sensor is impractical in the field; it can take a maximum of $1 \mathrm{~kg}$ pressure, and could easily be damaged. We used it to detect the moment someone placed a bag of books on top of it, to simulate the placement of a bag-bomb type of IED.

\section{What we tested and how we set up}

The experiment detailed here took place on September 17, 2010 and was set up by myself, with the assistance of Riqui Schwamm. The experiment was video taped, and the recording can be found with the media attached to this thesis. I was the human subject walking through the experiment.

Each laptop was set up to record sound, and 7 were set up with sensors. The program to collect sensor data was written by myself, and is attached in the appendix. The data collection program polls the PhidgetInterfaceKit to report raw sensor values from each of the attached sensors. The data was filtered using Matlab, using threshold values. Therefore, all of the "uninteresting" data points were taken out. The threshold values were set after careful experimentation with the sensors; when a threshold is reached it is generally 2 standard deviations away from the regular values reported by the sensors when nothing is in view. However, the threshold values are as follows:

\begin{tabular}{|l|l|l|}
\hline Sensor & threshold & notes \\
\hline IR Motion & 500 away from arithmetic mean & change $>500$ works well \\
\hline IR Distance & 100 away from arithmetic mean & indicates high prob. of detection \\
\hline Sonar & select tuples below 250 & this is the $<1$ yard range \\
\hline Precision Light Sensor & 300 away from arithmetic mean & indicates high prob. of detection \\
\hline
\end{tabular}

Having thresholds eliminates false-positives. We are highly confident that a subject is in range when a sensor reports a raw value beyond this threshold. 


\section{False negatives}

Briefly, the issue of false-negatives should be discussed. It may be possible to "sneak up" on a sensor. If this is the case, it has been noted in the previous section detailing the functionality and limitations of the sensor. For example, the infrared motion sensors monitor changes in fields and are more effective when a subject is moving across the field. Experimentation showed the sensor's detection range was reduced on a cold, foggy day when a subject walked directly toward the sensor. Thus a false-negative was reported (the sensor didn't report motion when a subject was indeed within range). 


\subsubsection{Experiment Plan}

In discussions with Dr. Neil Rowe he suggested the following plan for the experiment. Please note the type of suspicious behavior (or control) each experiment attempts to produce is in bold. Experiments were video taped by placing a camera on the third floor of Glasgow Hall East (GE) at NPS. Video was used to achieve ground truth for subjects walking through the course. Microphones were a separate part of the experiment, not part of this thesis yet important to the overall research. Please see paper [34].

1. One subject walks straight across the sensor field from sensor 4 to sensor 8 without stopping from bottom to top of the map, then back again. [control]

2. The subject walks from sensor 4 to the center of the sensor field and makes a left turn, then continues past sensor 9. [control]

3. The subject carries a bag and walks from sensor 4 to the center of the sensor field and makes a left turn, then continues to a position near sensor 6 where there is a force sensor run by 6 , then places the bag on the force sensor. They then walk past sensor 9. [loitering, object placement]

4. Similar to step 3 except the subject picks up the bag and carries it off to the left past sensor 9. [loitering, object placement]

5. The subject walks to the center of the sensor field, then to the area of sensors 5-7, and loiters within a circle of a ten foot radius for 30 seconds, then walks off past sensor 9. [meandering, improper location]

6. Similar to step 5 except the subject loiters within a twenty foot radius. [meandering, improper location]

7. The subject walks to an area of bare ground near sensor 5 with a shovel and inserts the shovel five times into the ground. [loitering, digging (for benefit of microphones)]

8. One subject walks to the center of the sensor field, and then to the area of 5-7, and loiters within a 10 foot radius. Then another subject walks across the sensor field from top to bottom, starting when the first subject starts loitering. [loitering, meandering, tracking with microphones] 
9. One subject walks to the center of the sensor field, and then to the area of 5-7, and loiters within a 10 foot radius. Then another subject walks across the sensor field from right to left, starting when the first subject starts loitering and passing the first subject. [loitering, meandering, tracking with microphones]

10. One subject drags an object from the bottom of the sensor field to the top (sensors 4 to 8 ). abnormal movement, tracking with microphones]

\subsubsection{Analysis of sensors}

The graphic marked Layout shows the placement of laptops with Phidgets sensors connected in the quad area at the Glasgow Hall building on the NPS campus, Figure: 4.9. Created by N. Rowe 


\section{Layout}

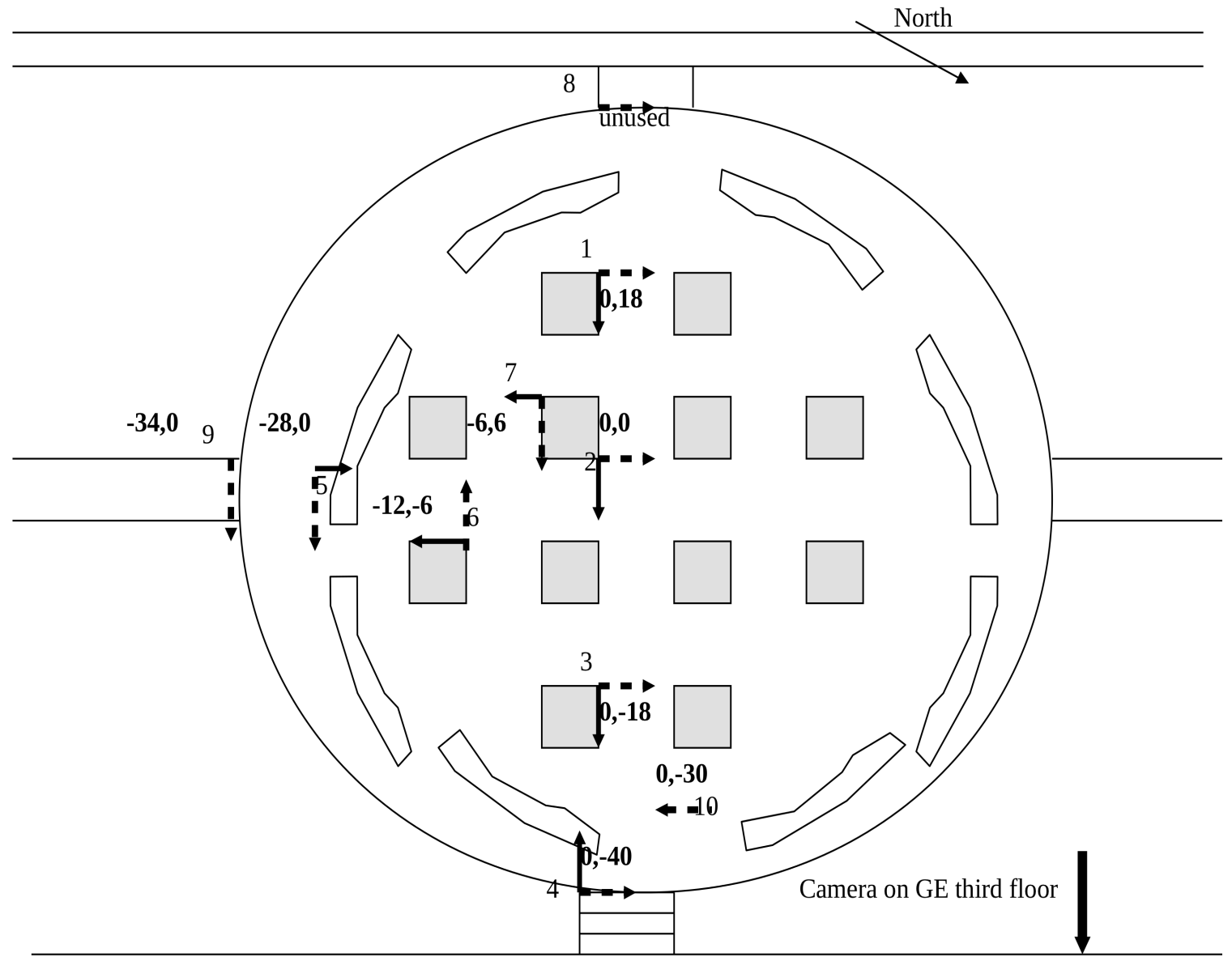

Numbers are sensors. Laptops 1-7 are Phidget sensors with infrared, motion, light (filtered), sonar, and microphone. Lap 6 also has a force sensor. 8-10 are microphones only. Microphones and sonar should be oriented with the solid arrows. narrow-infrared with the dotted arrows.

Notes: During experiment, Laptop 8 was not operational. Laptop 6 was missing a light sensor but had a force sensor.

Figure 4.9: placement of sensors in Glasgow Hall quad 


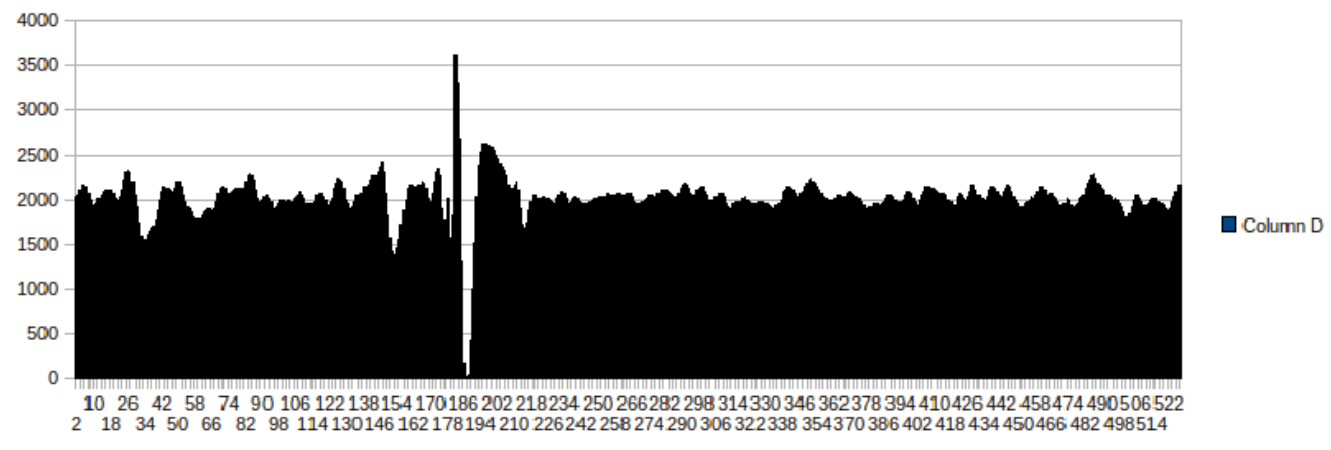

Figure 4.10: normal behavior, infrared motion sensor

\section{Generalized Data Analysis:}

We can make a quick judgment about suspiciousness by comparing two graphs. Simply put, a normal result will show a simple spike where a person walked past, and suspicious behavior such as loitering or meandering will show clustering behavior with many spikes. In the following example we will look at data from experiment 2 which was normal behavior and compare it with experiment 3 which had suspicious activity, where a bag was placed at location 6 .

The first example shows normal behavior from a person walking past laptop \#6, Figure: 4.10. This is simply a graph of one column of the raw data file collected from the experiment; this happens to be Column D, the Infrared motion sensor. The graph shows one spike and dip as the human subject walked past. The normal behavior in this experiment was to walk straight past location six without stopping.

The second example shows suspicious behavior at laptop \#6, Figure: 4.11 . In experiment 3 the subject stopped to place a bag on the ground near this laptop. The subject stayed in the area for approximately 10 seconds as the bag was placed. The infrared motion sensor has several peaks and valleys beyond a threshold of 500 raw data units. This indicates detection of the subject and suspicious behavior. We can make a simple analysis by comparing the two graphs: A few spikes is normal and many spikes is suspicious. The clustering of peaks is a trigger for the loitering metric.

Graphing raw data is a fast way for the human eye to determine if suspicious behavior is occurring. Tracking a subject is possible with even simple, binary sensors. The next example shows how 


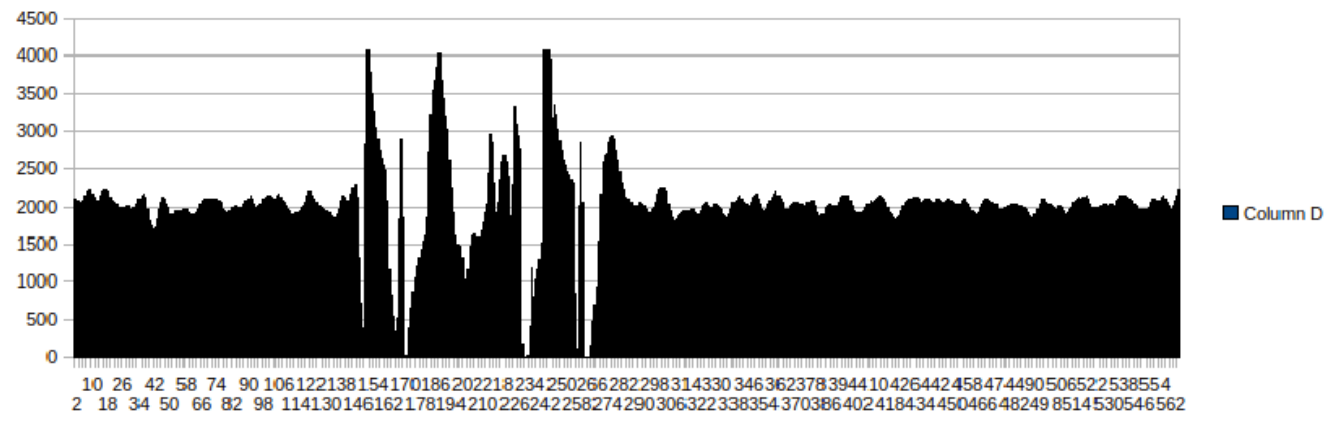

Figure 4.11: suspicious behavior detected with infrared motion sensor

the infrared motion sensors can be used to track a subject walking through the sensor field, seen in Figure: 4.12. Here, a single person walks from locations 4, 3, 2, 1 and back 1, 2, 3, 4. This graph demonstrates that infrared motion sensors can be used to track an individual which corroborates our experiment findings from the 2010 paper [32].

Similarly the infrared distance sensors can be used to track an individual. What is most interesting is that they can also be used to detect objects placed in the area. In this experiment, I was digging at a location and then I left the shovel there. The graph in Figure: 4.13 demonstrates the metric of Leaving An Object. The IR distance sensor reports the new object as a long streak.

The sonar range sensors did not perform so well. The data was noisy and had repeated echos as seen in Figure: 4.14. These show up as regular 'blips' on the graph where data points were reported beyond the selected threshold, yet nobody was at the location reporting "interesting" tuples. The long streaks indicate ground reflection, or the close proximity of an object in the field around the sensor. These do not indicate leaving an object, since they appear from the beginning of the experiment when the area had no activity. This indicates the need for tuning the sonar sensors individualy.

The light sensors worked surprisingly well, as shown by Figure: 4.15. It was a bright day, with distinct shadows. Again in the digging experiment, the light sensor detected my digging. It was so accurate that it recorded 5 clusters of data because each time I used the shovel, I leaned forward and my shadow crossed the sensor: 


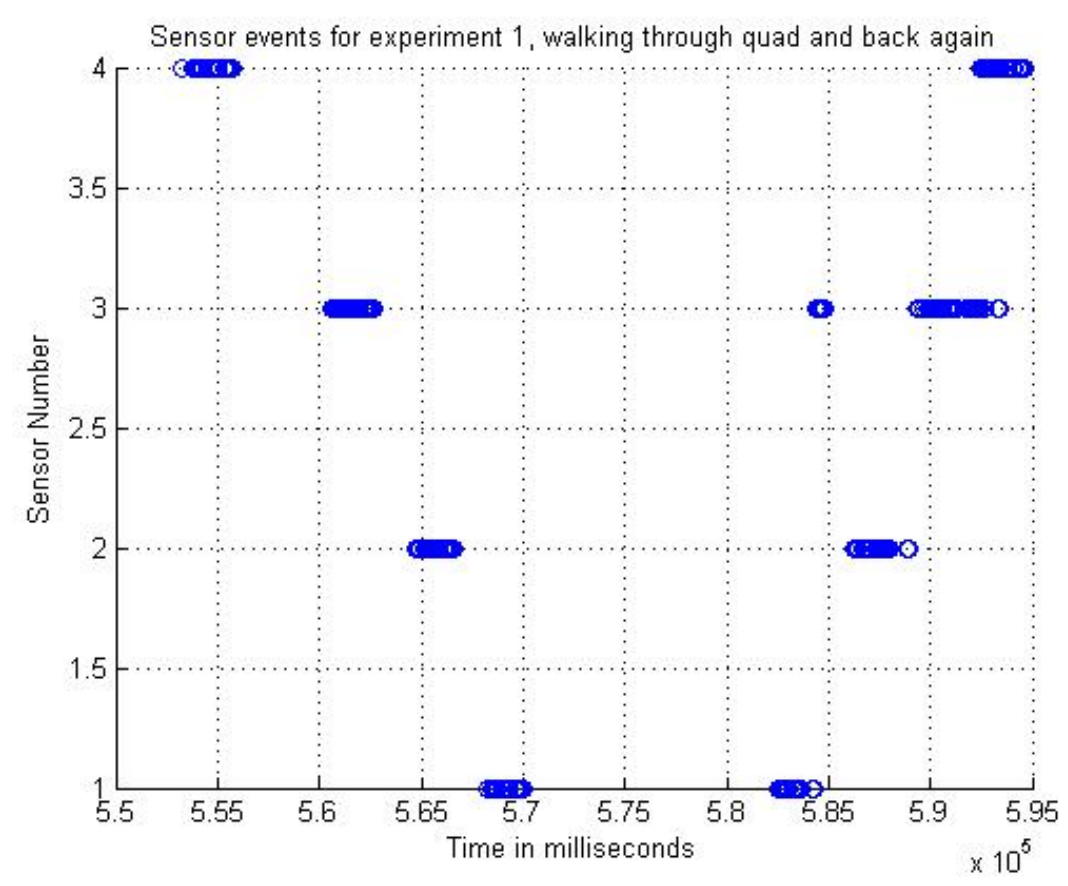

Figure 4.12: Normal behavior, tracking an individual walking

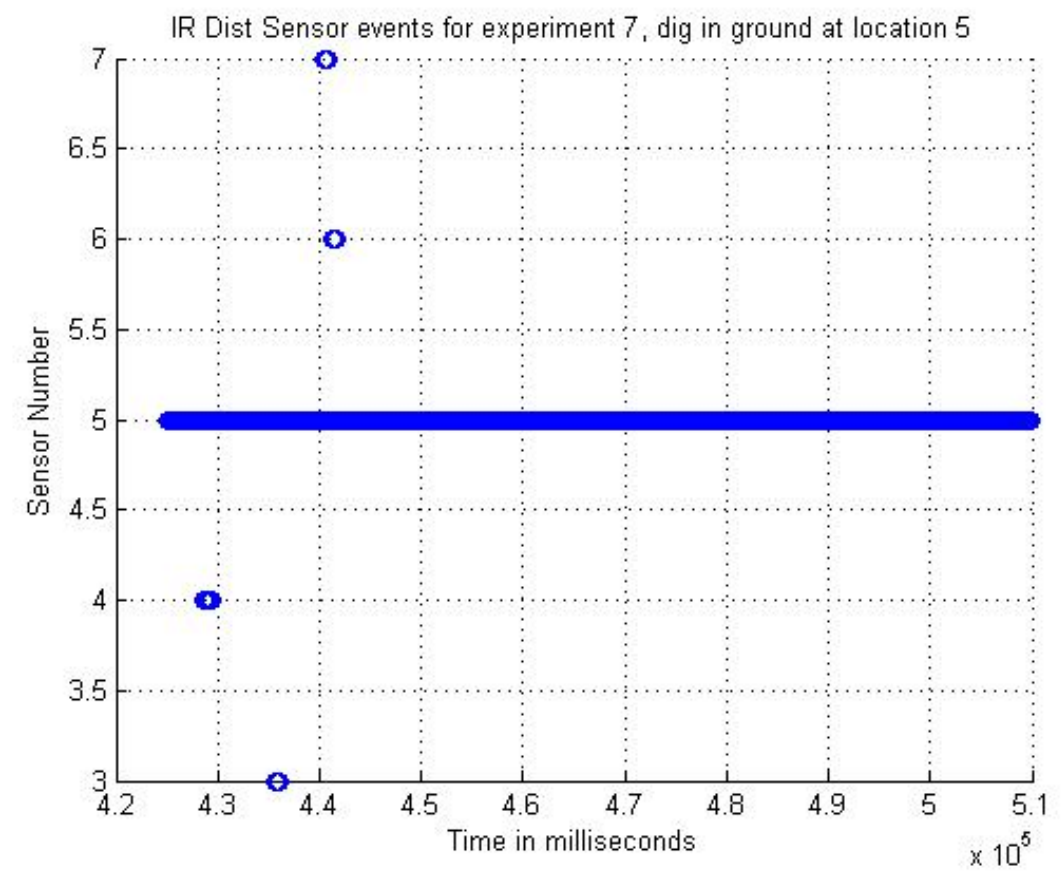

Figure 4.13: suspicious behavior, object left in front of sensor 


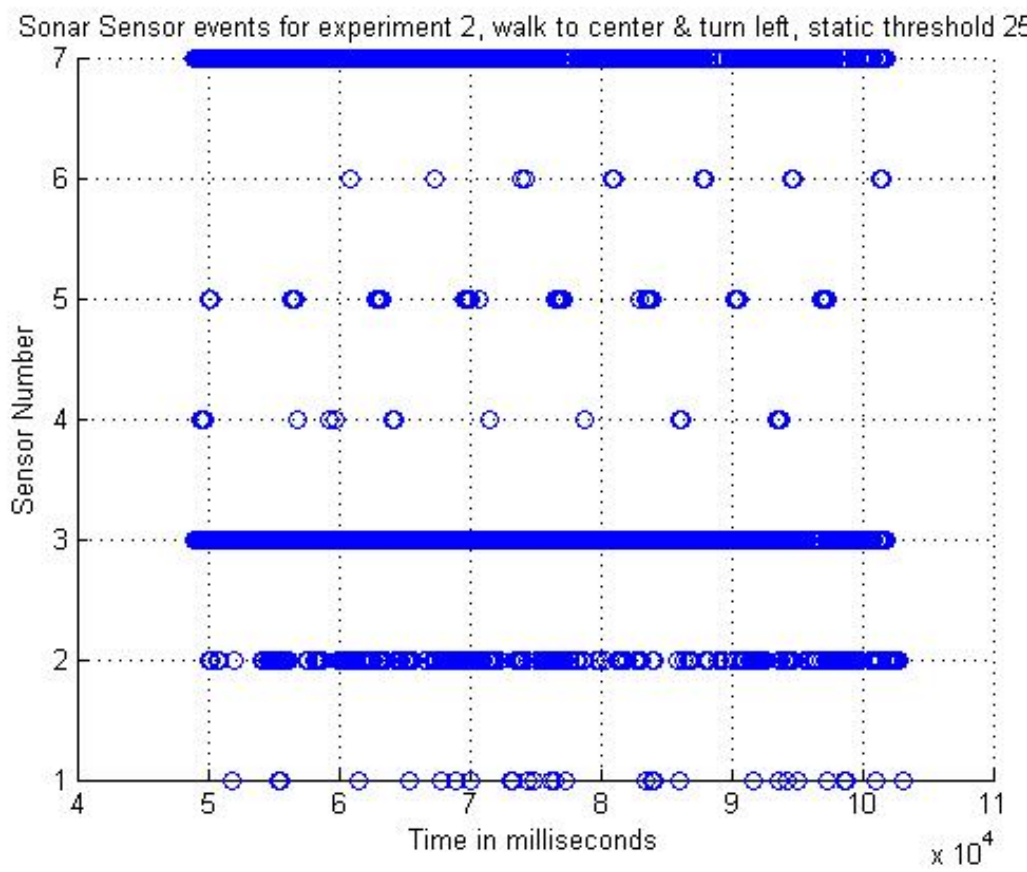

Figure 4.14: noisy sonar sensors reporting false positives

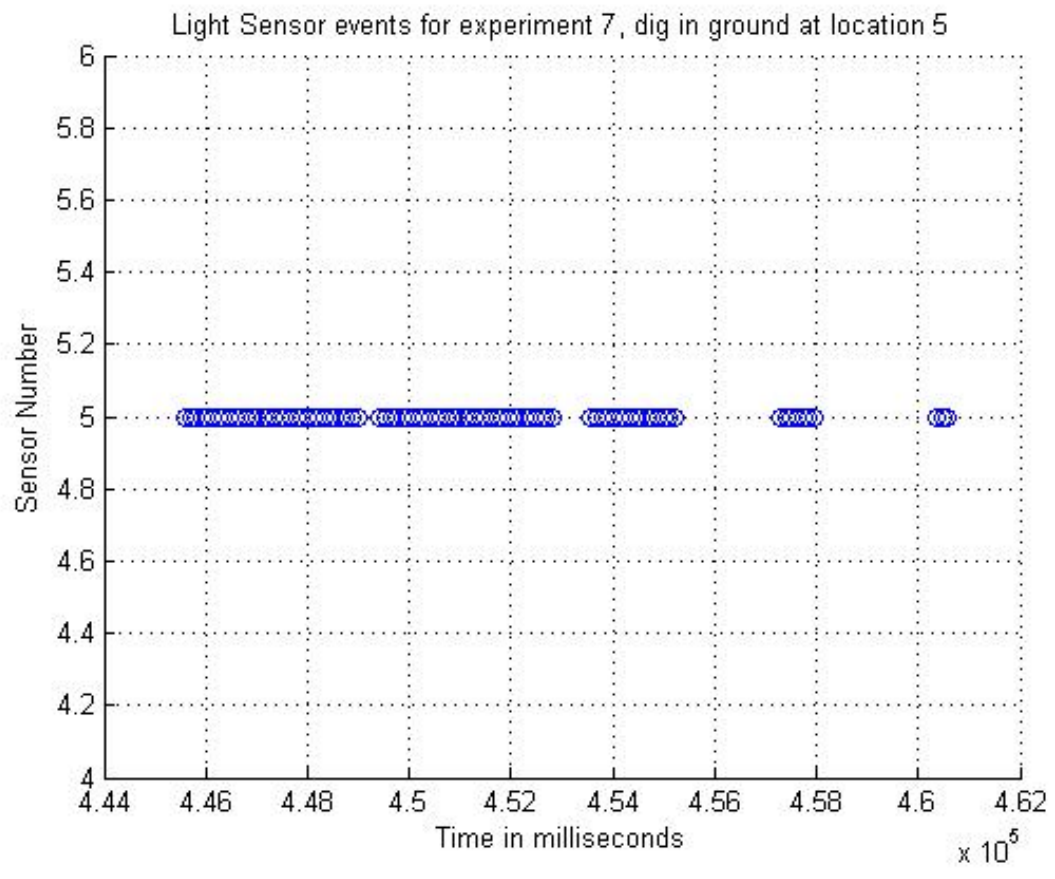

Figure 4.15: light sensors detect suspicious behavior 


\subsubsection{Quick summary of sensor performance}

\section{How well did each sensor perform?}

Infrared Motion Sensors: They worked great. The results reproduce the successful outcome of our experiments in Summer 2009 using the Crossbow Motes with the IR motion detectors. This shows that IR motion sensors are good at detecting suspicious behavior. However there are certain conditions where they should not be used, for example, placing them underneath a tree with limbs that might blow in the wind will cause all kinds of erratic datapoints. You are basically detecting the wind, not suspicious people.

Infrared Distance Sensors: These are great at telling you when someone was at a precise location. They are helpful in tracking. Clustering of data definitely shows suspicious activity. For example, you wouldn't cross the door beam in a retail store several times in a row, just to make the door sensor go "bing!" -unless you happen to be 4 years old and think that kind of thing is fun. It's not normal adult behavior to go in and out the door repeatedly or play with the sensor. That activity indicates loitering in the area anyway.

Sonar Range Finders:These are not all that great, because the sensors are noisy and they have a long range (5 yards). In my initial graphs I used a threshold that was too low and all I got was noise. The threshold has to be set quite high. For example, the paths in the quad were 6 feet wide. Therefore a threshold should be used that brings the effective distance of detection down from 25 feet to 6 .

If anything, I would have to say the Sonar Sensor did not work. Without data averaging, the raw data values plotted at a very high threshold (only data showing 1 yard proximity to the sensor) showed regular noise. Averaging of data had to be done to further analyze the results.

When data is plotted by taking the average of 10 readings per second, we find no useful clusters to represent a subject in the field. We simply get noise or no data at all. Averaging may also be a bad solution because of subject speed. It may take a subject less than 1 second to move through the cone of view for a sonar sensor. The sensors are more suitable for home robotics experiments, where a robot (like a Roomba for example) is trying to keep from running into the wall.

Light Sensors: These surprised me. They are very much binary sensors. They simply detect when your shadow falls across the sensor. Repeatedly activating the light sensor indicates loitering. All the graphs looked pretty good; the sensor didn't report anything during the non-suspicious ac- 
tivity. They only reported during the experiments when a subject was loitering. It is therefore good that many of the graphs do not exist-no data points were selected in the threshold program-because nothing suspicious was going on. Having no alarm during the non-suspicious tests is good.

\section{Table of test results}

\begin{tabular}{|l|l|l|}
\hline Sensor & Success in prototypes & Ranking \\
\hline Infrared Motion & success & first place \\
\hline Infrared Distance & success & second place \\
\hline Light sensors & success & third place \\
\hline Sonar sensors & failure & last place \\
\hline
\end{tabular}




\section{Chapter 5}

\section{Conclusion and future work}

\subsection{Strengths and Weaknesses of this Work}

Good things about this experiment

- Learn By Doing experiments

- work with real COTS products

- demonstrated cheap sensors do work for this application

Some weaknesses

- sonar sensors need more work to adapt to test environment

- different testbed, such as a straight line dirt road

- networked system to put everything together in real time

\section{Good things about this experiment}

\section{Learn By Doing experiments}

The work with Phidgets sensors followed a rapid development programming style as we developed software that polled the sensors for data. Also, our experiment design evolved as we learned more about the sensors. Performing black-box testing and reverse engineering the product specifications allowed us to develop placement of our prototypes. We did several trial-runs of the prototype experiment in the Quad at Glasgow Hall; each time we learned more about the sensors themselves. 
Black-box testing and prototype development occurred simultaneously, and we learned more about the sensors each time. Developing tests on the fly was a great way to practice rapid development. New software methods could be written and added (such as a noise filter) when we found the need.

\section{Work with real COTS products}

We demonstrate the successful hypothesis that commercial off the shelf products should be used by working with them directly. Cheap sensors do work for this application. Current practices in the military-and with military contractors-is to go with expensive development methods and expensive specialized equipment. At the time of writing it is generally accepted that the problem can not be solved in an inexpensive way. We demonstrate our own proof of concept by purchasing COTS hardware and using it in a way that does not involve complex engineering or military clearance levels. Everything we did is academic; it can be done by others who purchase the same equipment.

\section{Demonstrated cheap sensors do work for this application}

Anyone can purchase Phidgets sensors (or similar COTS hardware) perform experiments with them and analyze the results. The graphic results are easy to see in a widely available mathematics suite like Matlab. The data patterns that emerge when subjects perform suspicious activity can be visualized with graphs. Further development can be done to write algorithms in software that basically do the same thing we do by eyeballing a graph. We will leave that automation for future research. What we have shown is that four types of suspicious behavior can be detected with analysis of simple sensor data.

\section{Some weaknesses}

\section{Sonar sensors need more work to adapt to test environment}

It was not obvious during black-box testing that the sonar sensors would perform so poorly in field experiments. Black-box testing generally involved a stationary sensor and a stationery subject. The sensors are designed to be placed on a slow-moving object such as a home-built robot, which then looks at a stationary object. We found through experimentation that we were using them in reverse: Our sensors were stationary and the subject was moving. They do not appear to be made to detect passing objects. They are better suited to keeping a robot from running into a large object like 
a wall. Thus, the sonar sensors are great for indoor use, but not intended for outdoor use. They require fine tuning for each sonar sensor placed at each location. There were too many ground reflections from the environment that caused regular interference. Further work is needed to make the sonar range finder useful for the purpose of detecting suspicious behavior in the same way that the IR distance sensor did.

\section{Different testbed, such as a straight line dirt road}

Different testbeds would allow for exploration of different types of prototype systems. For example, we studied IED placement in a public area with crossing pathways. A crossroads in Iraq could be simulated, with different ground environments (i.e. compacted soil vs. paved walkways.) A dirt road would be more in keeping with the types of terrain that military Route Clearing Patrols encounter. There also exists some weakness in the types of sensor available. For example, we would have liked to incorporate some kind of magnetic induction field loop, such as the type placed at paved city intersections to detect cars waiting at a light. Unfortunately these do not exist in a Phidgets sensor, although the publicly installed versions are in the low hundreds to procure. Again unfortunately, they are meant for paved roads and are highly sensitive to placement depth. Such a thing does not yet exist for dirt roads. Similarly there are no inexpensive pressure strips suitable for deploying along a dirt road. Such sensors are highly delicate; the Phidgets sensor was a piezo-electric plastic ribbon approximately 1 meter long.

\section{Networked system to put everything together in real time}

A prototype system using MicaZ Motes would be beneficial to collect data in real time. Work could be done to analyze data in real time. Automated software to detect suspicious behavior is possible. [1] However the task is possibly larger than a Master's Thesis would involve, owing to time and overall complexity of a large-scale and fully-functional prototype.

\subsection{Conclusions}

The main goal is to detect suspicious behavior. Since it would be nearly impossible to solve the complete problem, and nearly as difficult to define it, we derived four requirements from the goal of detecting suspicious behavior. Those derived requirements are to detect loitering, meandering, 


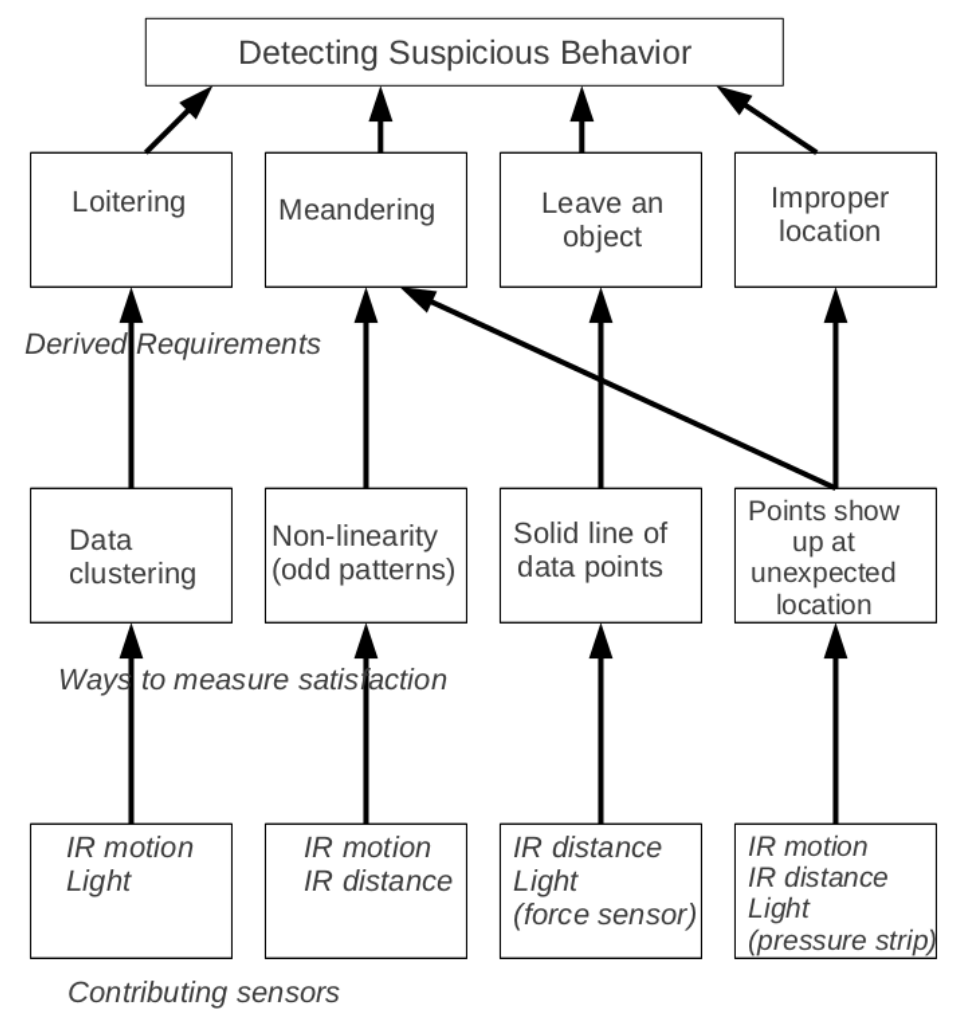

Figure 5.1: developing links between sensors and detecting types of suspicious behavior

leaving an object and improper location. Metrics were developed in order to do that, and then ways of looking at raw data from sensors was linked to which metrics those data patterns contributed to. In essence, my contribution is the arrows on the chart in Figure: 5.1. I linked specific sensors to patterns of data, and using simple software techniques I determined metrics that would satisfy our four derived requirements.

\subsection{Future work}

\section{Prototype Wireless Sensor Network}

A prototype Wireless Sensor Network should be built. We have shown it is possible to detect suspicious behavior with cheap sensors, so a prototype needs to be deployed in the field. Perhaps in the future some military funding can be devoted to building a field-suitable, deployable prototype, 
as well. As seen in Bordeau [4], the military is looking for ways to cut costs and use COTS hardware.

\section{Deployment in the Environment}

Work should be done that examines how to deploy sensors in a live environment so they won't be tampered with. Can the sensors be made an "invisible" (or ignored) part of the background environment? One scenario may be the development of a smart mile marker. This is similar to a mile-marker as deployed by CalTrans all along California highways. They use sturdy steel posts embedded into the ground, and a steel sign with reflectors that is designed to mark distances. Future work in solar cells could incorporate reflectors and solar cells together so the mile markers could power themselves. The steel construction lends itself to sturdiness and weather resistance. Somewhere in the structural post, electronics may be housed for the sensors and wireless Mote. Armor and tamper-resistance need to be factored into any design. If the Motes are camouflaged, armored housings may interfere with the sensor's ability to gather data. For example, the acoustics will surely be hindered if a Radio Shack mic is placed inside a metal box.

\section{Limitations of Wireless Sensor Networks}

Wireless sensor networks can't handle streaming video or audio. They might be able to send 10 seconds of sound, or a single CCD snapshot of an area. Future work in bandwidth limitation may expand the network's ability to handle larger message packets. One of the nice things about using low-cost sensors is that binary sensors like infrared motion detectors are less invasive to personal privacy than video monitoring.

\section{Other sensors}

We would have liked the Phidgets Vibration Sensors to detect footsteps. Sadly, they were unable to do so because of their simple design. They only detected vibration when the object they were mounted to was vibrating. The only use we could see for this is to detect when a bridge is vibrating due to vehicle or pedestrian traffic, where the sensor needs to be affixed securely to the bridge. Placing the sensor on the ground would only be useful in detecting an earthquake. Heavy footsteps-even 
stomping-inches away from the sensor do not register with the simple piezo-electric device.

The Phidgets magnetic sensors annoyed us for their unclear labeling and inability to detect magnetic objects. We anticipated they would behave like the "magnetic sensor" in the Crossbow MSP410 Mote, that is, they would detect metal objects. Instead they were only useful in detecting magnets placed directly on the device. This might be useful if terrorists attached IEDs to road signs using magnets, but that does not seem likely. We did not see the usefulness of being able to detect a refrigerator magnet (as were included in the package) except in some hobby or home robotics project.

A CCD camera could be useful, if triggered by some other sensor. This is similar to the automatic tree-mounted cameras that deer hunters use to detect patterns of animals feeding in a forest. Having a CCD camera to quickly photograph or video a suspicious person may bring near-instant results in determining if motion detected in the area is suspicious. In an example more closely related to Afghanistan: A vehicle is stopped on the side of the road (loitering behavior) and it brings up the question whether the occupants are simply changing a flat tire or unloading digging tools and explosives. Once loitering is detected, an automated system may choose the closest camera to take a 4 second video (short time minimizes network packet load) and deliver it to a monitoring station. Flagging the packets with a suspicious behavior warning will indicate to security staff that the video needs to be checked out urgently. This follows the proactive system requirements explained earlier.

As long as science fiction exists, people will dream of new technology. Other sensors will surely be developed in the future that fit the low cost requirement or this thesis. Light Detection and Ranging (LIDAR) is currently being integrated into wireless sensor networks for surveillance applications. Progress in radar and laser range-finding technology (such as LIDAR) may bring the costs down to the point they can be included in a cheap wireless sensor Mote. Currently researchers are working around the problems of radar and LIDAR being expensive and are building prototypes of of low-cost $3 \mathrm{D}$ perception sensors [25]. 


\section{Bibliography}

[1] Peter Bak, Christian Rohrdantz, Svenja Leifert, Christoph Granacher, Stefan Koch, Simon Butscher, Patrick Jungk, and Daniel A. Keirn. Integrative visual analytics for suspicious behavior detection. In IEEE Symposium on Visual Analytics Science and Technology, October 2009. VAST 2009 Challenge.

[2] Daniel Barbara, Carlotta Domeniconi, Zoran Duric, Maurizio Filippone, Richard Mansfield, and Edgard Lawson. Detecting suspicious behavior in surveillance images. In 2008 IEEE International Conference on Data Mining Workshops. IEEE Computer Society, June 2008.

[3] Shashank Bengali. Cost-cutting will be tough for military, gates predicts departing defense chief foresees smaller force, overhaul of benefits. Houston Chronicle, page 9, May 252011.

[4] M. Boudreau. Acoustic rapid cots insertion: A case study in modular open systems approach for spiral development. In System of Systems Engineering, 200\%. SoSE '07. IEEE International Conference on, pages $1-6$, april 2007.

[5] Army Pfc. April Campbell. Face of defense: Route-clearance soldier leads from front. http://www.defense.gov/news/newsarticle.aspx?id=49572, April 2008. Internet based news source: www.defense.gov.

[6] Michele Chabin. Jerusalem blast stirs terror fears. USA Today, page News 7a, March 24, 2011.

[7] Varun Chandola, Arindam Banerjee, and Vipin Kumar. Anomaly detection: A survey. ACM Comput. Surv., 41:15:1-15:58, July 2009.

[8] Christopher Alan Day. The design of an efficient, elegant, and cubic pico-satellite electronics system. Master's thesis, California Polytechnic State University, 2004. 
[9] Simon Fong and Zhuang Yan. A security model for detecting suspicious patterns in physical environment. In Third International Symposium on Information Assurance and Security. IEEE, July 2007.

[10] Jason Ford. Border patrol. online news article: http://www.theengineer.co.uk/news/borderpatrol/303364.article, November 2007. Web-based news source: www.theengineer.co.uk.

[11] Simone Gabriele and Paolo Giamberardino. Redundant coverage for noise reduction in dynamic sensor networks. WTOS, 7:855-865, October 2008.

[12] Robert Gates. Speech to the american enterprise institute. American Rhetoric Online Speech Bank, May 24, 2011. http://www.americanrhetoric.com/speeches/robertgatesamericanenterpriseinstitute.htm.

[13] Dave Hecht and Samatha Truelove. Ied hunters. http://www.youtube.com/user/ISAFMEDIA, August 2009. Produced by ISAF Public Affairs / STRATCOM.

[14] Jan M.H. Hendrickx, Nicole Alkova, Sung ho Honga, Remke L. Van Damb, Jan Kleissla, Heather Shannona, John Measona, Brian Borchersa, and Russell S. Harmon. New mexico tech landmine, uxo, ied detection sensor test facility: Measurements in real field soils. SPIE, April 2006.

[15] House of Representatives, One Hundred Eleventh Congress. Defeating the improvised explosive device (IED) and other asymmetric threats: Reviewing the performance and oversight of the Joint IED Defeat Organization (JIEDDO), number H.A.S.C. No 111-105. U.S. Government Printing Office, October 2009. Hearing held October 29, 2009.

[16] Crossbow Inc. Msp410 specification sheet. Technical report, self published, 2005.

[17] John Keller. Industry and government prepare counter-attacks against electronic parts counterfeiting. Military $\&$ Aerospace Electronics, 21(4), April 1, 2010. Technology Focus.

[18] Gerard Maral, Michel Bousquet, and Zhili Sun. Satellite Communications Systems: Systems, Techniques and Technology. John Wiley \& Sons, Ltd., fifth edition, 2009.

[19] MetaVR. Product informaton. http://www.metavr.com/products/products.html, 2011.

[20] Matthew OHara. Detection of ied emplacement in urban environments. Master's thesis, U.S. Naval Postgraduae School, September 2008. 
[21] Gary L. Powell, Louis A. Tyska, and Lawrence J. Fennelly. Casino Surveillance and Security: 150 Things You Should Know. Asis International, 2003.

[22] Ferdinand Protzman. Head of top west german bank is killed in bombing by terrorists. The New York Times, page A1, December 11989.

[23] J. Puig-Suari, C. Turner, and W. Ahlgren. Development of the standard cubesat deployer and a cubesat class picosatellite. In Aerospace Conference, 2001, IEEE Proceedings., volume 1, pages 1/347 -1/353 vol.1, 2001.

[24] QinetiQ. Optasense: Pipeline security and monitoring. Company product spec sheet: http://www.qinetiq.com/home_optasense/media/documentation.html, 2011.

[25] Sriranjan Rasakatla. Low cost 3d perception sensors. In ACM SIGGRAPH 2010 Posters, SIGGRAPH '10, pages 62:1-62:1, New York, NY, USA, 2010. ACM.

[26] K. Robert. Video-based traffic monitoring at day and night time. Technical report, NICTA Sydney, Road and Traffic Authority of New South Wales (RTA), 2006.

[27] N. Rowe, G. Singh, and X Chen. Improving localization accuracy using spatio-temporal information of objects. In 2010 IEEE International Conference on Communications (ICC), South Africa, May 2010.

[28] Neil C. Rowe. Detecting suspicious behavior from only positional data with distributed sensor networks. ASME Conference Proceedings, 2005(47438):2291-2296, 2005.

[29] Neil C. Rowe. Detecting suspicious behavior from positional information. In Proceedings of the Workshop on Modeling Others from Observatins at the International Joint Conference on Artificial Intelligence, Edinburgh, Scotland, July 2005. research sponsor: MOVES Institute, U.S. Naval Postgraduate School, Monterey, CA.

[30] Neil C. Rowe and Alex L. Chan. Rating whole-body suspiciousness factors in automated surveillance of a public area. In 2011 Intl. Conf. on Image Procesing, Computer Vision and Pattern Recognition, Las Vegas, Nevada, July 2011.

[31] Neil C. Rowe, Rey M. Osoteo, Riqui Schwamm, Cory R. Kirk, and Ahren A. Reed. Visualizing summaries of performance for instructors assessing physical-motion skills. In Proceedings of Global Learn Asia Pacific 2011, Melbourne, Australia, April 2011. 
[32] Neil C. Rowe, Ahren A. Reed, and Jose J. Flores. Detecting suspicious motion with nonimaging sensors. Advanced Information Networking and Applications Workshops, International Conference on, 0:991-996, 2010.

[33] Neil C. Rowe, Jonathan Roberts, and E. John Custy. Automated assessment of suspiciousness of people in public spaces from path tracking. 2005.

[34] Neil C. Rowe, Riqui Schwamm, Jeehee Cho, Ahren A. Reed, Jose J. Flores, and Arijit Das. Detecting explosive-device emplacement at multiple granularities. In Proceedings of the Military Sensing Society (MSS) National Symposium, Las Vegas, Nevada, July 2010.

[35] Herbert A. Simon. The Sciences of the Artificial. The MIT Press, 1996.

[36] Phillip D. Stroud. A recursive exponential filter for time-sensitive data. Technical report, Los Alamos National Laboratory, 1999.

[37] Xiuhong Sun and William Chen. A dual-swath buckeye eo imaging system and its applications for emergency response to natural disasters. In IGARSS 2009. IEEE, 2009.

[38] Joshua Sundram and Phua Poh Sim. Using wireless sensor networks in improvised explosive device detection. Master's thesis, U.S. Naval Postgraduate Shool, 2007.

[39] Benjamin Weiser and Eric Schmitt. Scientist tied to al qaeda is ordered held without bail. New York Times, Late Edition (East Coast), page A.12, August 62008.

[40] Brian Wood, Nelson Irvine, Gordon Schacher, and Jack Jensen. Joint multi-mission electrooptic system (jmmes) report of military utility. In Joint Multi-Mission Electro-Optic System (JMMES) report of military utility, march 2010. 


\section{Appendix A}

\section{Phidgets black box testing}

These tests were done to determine the range and performance of the sensors before we built our prototypes for testing in the quad at Glasgow Hall. [Note: Most analysis writeups are complete. There are still a few that are not, however the basic information is there.]

Experiment 1 30, June, 2010

Sensors: Phidgets vibration sensors

Field: Top of Spanagel Hall, sensors on 0 yard mark, marks measured out every 1 yard away to 10 yds.

Conditions: Windy 15-18 mph. Temperature 63 deg. F.

Purpose: Working with vibration sensors

Program: java Sensortest 473

Implementation: Three vibration sensors placed together on a 10 yard course. 1. vibration sensor on ground. 2. vibration sensor on wood block. 3. vibration sensor mounted inside an Altoids tin. Every 30 seconds walk past sensors. Start at 0 yards, directly in front of sensors. Each 30 seconds move 1 yard back and walk past sensors again. Continue until 10 yard mark is reached.

Analysis: It does not appear that any vibration from footsteps was detected. The first two sensors, one taped to the ground and another mounted on a wooden block placed on the ground seem to give the same kinds of readings all the time. There is no detectable change between subjectwalking and subject stationary. The third sensor, mounted in an Altoids tin remained relatively quiet. There are potentially two problems with this experiment: 1 . The number of samples per second is too small. 2. The sensors are being blown by the wind, which was about $15 \mathrm{mph}$ on top of Spanagel Hall. 


\begin{tabular}{|r|r|r|r|r|r|r|}
\hline & ave 0 & ave 1 & ave 2 & stddev 0 & stddev 1 & stddev 2 \\
\hline 0 yds & 2005.05 & 1962.39 & 2004.5 & 52.93 & 42.38 & 7.56 \\
\hline 1 yds & 2016.09 & 2014.76 & 2001.75 & 55.81 & 48.44 & 2.56 \\
\hline 2 yds & 2062.37 & 2054.04 & 2061.3 & 78.92 & 129.84 & 2.43 \\
\hline 3 yds & 2055.81 & 2017.45 & 2059.82 & 69.36 & 87.62 & 3.1 \\
\hline 4 yds & 2031.14 & 2004.35 & 2050.06 & 83.34 & 102.66 & 2.2 \\
\hline 5 yds & 2006.7 & 2019.1 & 2044.37 & 40.8 & 53.1 & 1.83 \\
\hline 6 yds & 2018.86 & 2011.31 & 2040.87 & 41.16 & 61.52 & 1.65 \\
\hline 7 yds & 2037.33 & 2013.92 & 2034.79 & 52.92 & 87.22 & 2.81 \\
\hline 8 yds & 2025.73 & 2003.67 & 2036.23 & 54.08 & 65.94 & 2.84 \\
\hline 9 yds & 2071.66 & 2050.18 & 2042.5 & 83.7 & 92.74 & 4.41 \\
\hline 10 yds & 2035.95 & 2052.94 & 2041.88 & 62.37 & 91.33 & 3.84 \\
\hline
\end{tabular}

\begin{tabular}{|r|r|r|r|r|r|r|}
\hline & $\min 0$ & $\min 1$ & $\min 2$ & $\max 0$ & $\max 1$ & $\max 2$ \\
\hline 0 yds & 1909 & 1881 & 1994 & 2177 & 2078 & 2022 \\
\hline 1 yds & 1885 & 1937 & 1998 & 2151 & 2134 & 2006 \\
\hline 2 yds & 1932 & 1875 & 2053 & 2343 & 2590 & 2066 \\
\hline 3 yds & 1905 & 1873 & 2054 & 2274 & 2194 & 2066 \\
\hline 4 yds & 1869 & 1888 & 2046 & 2275 & 2445 & 2057 \\
\hline 5 yds & 1917 & 1902 & 2042 & 2102 & 2186 & 2046 \\
\hline 6 yds & 1881 & 1877 & 2038 & 2125 & 2137 & 2043 \\
\hline 7 yds & 1905 & 1857 & 2030 & 2186 & 2234 & 2041 \\
\hline 8 yds & 1929 & 1873 & 2030 & 2158 & 2170 & 2040 \\
\hline 9 yds & 1921 & 1901 & 2030 & 2254 & 2309 & 2050 \\
\hline 10 yds & 1893 & 1861 & 2036 & 2210 & 2258 & 2056 \\
\hline
\end{tabular}


Experiment 2 30, June, 2010

Sensors: Phidgets vibration sensors

Field: Top of Spanagel Hall, sensors on 0 yard mark, 1 yard circle marked around sensors.

Purpose: Test accuracy of vibration measurements

Program: java Sensortest 253

Implementation:

Sample at $2 \mathrm{x}$ per second. Walk in a circle at 1 yard away every 30 seconds for three minutes.

Analysis:

The sensors do not seem to change when a subject is walking around them at close range. The two exposed sensors seem to pick up a lot of wind vibration, and the sheltered sensor reports little motion at all. Again, the sample rate may be too short, and the wind may be significantly affecting the exposed sensors. We are trying these sensors in order to compare them to a microphone, for the purpose of picking up footsteps. 
Experiment 3 2, July, 2010

Sensors: Phidgets vibration sensors (0) and IR distance (1) Field: Top of Spanagel Hall, sensors on 0 yard mark, marks measured out every 1 yard away to 10 yds.

Purpose: Test accuracy of distance measurements

Program: java Sensortest 2052

Implementation:

Walk in 1 yard circle every 30 seconds for 2 min. Sensors sample at 20x per second.

Analysis:

mote 0 - a lot of wind noise

mote 1 - a lot of wind noise

mote 2 - standard deviation is very small, this sensor detected nothing

It appears that the sensors are not detecting footsteps. There is no noticeable change when examining the data. The first two sensors are exposed to the wind and they only seem to pick up wind noise. The third sensor has been mounted inside an Altoids tin placed on the ground. This shelters it from the wind, yet it picks up nothing that can be correlated to footsteps. 
Experiment 4 1, July, 2010

Sensors: Phidgets sonar (0) and IR distance (1)

Field: Top of Spanagel Hall, sensors on 0 yard mark, marks measured out every 1 yard away to 6 yds.

Purpose: Test accuracy of distance measurements

Program: java Sensortest 582

Implementation:

Set up IR distance and sonar distance sensors. Walk in front of them every minute. Try this out to 6 yards. Sample rate is $5 \mathrm{x}$ per sec.

Analysis:

The data for the IR distance sensor appears sporadic. It seems to work out to 3 yards, but it misses the subject at 2 yards. The Sonar Sensor shows much more consistent results. We may judge whether or not the sensor detected an object by examining the standard deviations. During this experiment, both sensors saw nothing for close to a minute, then a subject walking past at a normal pace; the significant change in sensor reading gives a clear indication that the sensor detected the walking subject. Looking at the standard deviations between infinity-nothing in view-to subject in sight show dramatic changes in standard deviation. In the Sonar Sensor this change is over 100 Raw Value points. In the IR distance sensor this change is 50 Raw Value points.

The largest standard deviations for the IR range sensor come at 0 and 3 yards. Otherwise the readings seem to be all over the map. The standard deviations for the sonar distance sensor are more reliable-looking. If we go with a threshold-change of 500 Raw Value points, then the sonar sensor can see out to 4 yards or so. That threshold is not reached-but it comes close-at 5 yards. The manufacturer's spec sheet say that it can see this far, however we need to ask the question, can we trust the data. Setting a low threshold may give false positives since this is a rather noisy sensor. After all it is a microphone and it can pick up reflected and natural sound as well as it's own signal directly bounced off an object.

\begin{tabular}{|r|r|r|}
\hline & stddev SO & stddev IR dist \\
\hline start & 2.67 & 1.41 \\
\hline 0 yds & 262.9 & 237.21 \\
\hline 1 yds & 234.77 & 53.43 \\
\hline 2 yds & 236.52 & 9.61 \\
\hline 3 yds & 141.25 & 75.28 \\
\hline 4 yds & 37.45 & 19.14 \\
\hline 5 yds & 32.02 & 30.26 \\
\hline 6 yard & 2.75 & 4.39 \\
\hline
\end{tabular}

\begin{tabular}{|r|r|r|r|r|r|r|}
\hline & ave SO & ave IR dist & min SO & min IR dist & max SO & max IR dist \\
\hline start & 2018.63 & 18.27 & 2013 & 16 & 2026 & 21 \\
\hline 0 yds & 1981.26 & 45.89 & 56 & 16 & 2026 & 2234 \\
\hline 1 yds & 1985.23 & 24.56 & 232 & 16 & 2026 & 617 \\
\hline 2 yds & 1979.14 & 19.06 & 526 & 16 & 2026 & 181 \\
\hline 3 yds & 1996.73 & 22.81 & 822 & 16 & 2026 & 1320 \\
\hline 4 yds & 2014.03 & 19.29 & 1560 & 16 & 2026 & 349 \\
\hline 5 yds & 2014.2 & 19.99 & 1641 & 16 & 2026 & 541 \\
\hline 6 yard & 2018.79 & 18.45 & 2010 & 16 & 2026 & 91 \\
\hline
\end{tabular}


Experiment 5 Date: 2, July, 2010

Sensors: Phidgets sonar (0) and IR distance (1)

Field: Top of Spanagel Hall, sensors on 0 yard mark, marks measured every 1 yard away to 10 yds. Purpose: Test accuracy of distance measurements

Program: java Sensorfilter 552

Implementation: Stand directly in front of sensors. Remain stationary for 30 seconds then move 1 yard away. Start at 1 yard away and continue moving back to 7 yards distance.

Start: 15:33:00 (not in field)

$1 \mathrm{yd}: 15: 34: 00$

2 yd: $15: 34: 30$

$3 \mathrm{yd}: 15: 35: 00$

4 yd: $15: 35: 30$

5 yd: $15: 36: 00$

6 yd: $15: 36: 30$

7 yd: 15:37:00

(stand at 7 yards for $1 \mathrm{~min}$ )

Program stops automatically after 5 minutes.

\begin{tabular}{|r|r|r|}
\hline & stddev SO & stddev IR dist \\
\hline start & 2.64 & 1.46 \\
\hline 1 yds & 408.24 & 160.31 \\
\hline 2 yds & 114.47 & 51.2 \\
\hline 3 yds & 420.47 & 34 \\
\hline 4 yds & 309.1 & 3.92 \\
\hline 5 yds & 214.86 & 1.6 \\
\hline 6 yds & 21.09 & 1.46 \\
\hline 7 yds & 2.59 & 1.3 \\
\hline
\end{tabular}

Analysis of standard deviation shows that the sonar is noisy out to 5 yards. The IR distance sensor shows high standard deviation at close range, then settles down to very low after 3 yards. We hypothesize that the sonar range is 5 yards and effective IR distance range is 3 yards.

\begin{tabular}{|r|r|r|r|r|r|r|}
\hline & ave SO & ave IR dist & min SO & min IR dist & max SO & max IR dist \\
\hline start & 2018.67 & 18.54 & 2011 & 16 & 2026 & 22 \\
\hline 1 yds & 330.57 & 556.49 & 160 & 16 & 2019 & 665 \\
\hline 2 yds & 534.51 & 156.61 & 232 & 18 & 1495 & 587 \\
\hline 3 yds & 1270.46 & 45.21 & 514 & 16 & 2019 & 172 \\
\hline 4 yds & 1614.41 & 18.88 & 1145 & 16 & 2018 & 48 \\
\hline 5 yds & 1852.91 & 18.51 & 1429 & 16 & 2023 & 29 \\
\hline 6 yds & 2013.3 & 18.31 & 1881 & 16 & 2026 & 20 \\
\hline 7 yds & 2018.74 & 18.4 & 2010 & 16 & 2026 & 22 \\
\hline
\end{tabular}

The sonar sensor shows high readings in the 2000's when nothing is in view, and low numbers when an object is close. The sensor reported both maximum and minimum values during times when a subject was standing within range. This indicates the sonar sensor reports a lot of noise, with raw values that bounce around from highest to lowest. In order to detect an object, one must rely on the average sonar reading within a time period. The IR distance sensor is less noisy and shows significant readings out to 3 yards. The IR distance sensor shows low values when nothing is in range. 
Experiment 6 1, July, 2010

Sensors: Phidgets 1128 sonar sensor (0) and IR distance (1)

Field: Top of Spanagel Hall, sensors on 0 yard mark, marks measured out every 1 yard away to 10 yds.

Purpose: Test accuracy of distance measurements

Program: java Sensorfilter 1052

Implementation:

Sensors placed 4â̆ $\mathrm{I}$ high. Similar to previous experiment, this time starting at 7 yards and moving closer.

Synchronize stopwatch with start of test. Begin computer measurements at 15:42:00, at 15:43:00 appear in field at 7 yards, directly in front of sensors. Every 30 seconds move 1 yard closer to sensors. Leave the field after standing at the 1 yard mark for 60 seconds. End recording at 15:47:00.

Sonar sensor is Phidgets model 1128 - MaxBotix EZ-1 Sonar Sensor. Raw data values are around $2000+$ when nothing is in range, and values grow smaller as detected objects get closer. The spec sheet gives the following formula to translate raw values into distance:

Distance $(\mathrm{cm})=($ RawSensorValue $/ 4.095) * 1.296$

For exp 6, at 1 yard away, sensor reads 264 or $72 \mathrm{~cm}$. (2.36 feet). The error is 0.21 yards.

2 yards away, sensor $=556$ or $135.7 \mathrm{~cm}$ (4.45 feet). The error is 0.51 yards.

3 yards away, sensor $=999$ or $216 \mathrm{~cm}$ ( 7.08 feet). The error is 0.64 yards.

4 yards away, sensor $=1133$ or $276.7 \mathrm{~cm}$ (9.05 feet). The error is 0.98 yards.

5 yards away, sensor $=1145$ or $354.1 \mathrm{~cm}$ (11.61 feet). The error is 1.13 yards.

6 yards away, sensor reads 2021, the same as when nothing is in view for 15 yards. This is interesting because the product spec sheet (and the Phidgets web site) state that this sensor can see 6.45 meters, (approx. 7 yards.) 
Experiment 7 2, July, 2010

Sensors: Phidgets sonar (0) and IR distance (1)

Field: Top of Spanagel Hall, sensors on 0 yard mark, mark measured at 2 yards.

Purpose: Test accuracy of distance measurements

Conditions: windy, about $15 \mathrm{mph}$, temperature $61 \mathrm{deg}$. F.

Program: java Sensorfilter 1052

Implementation:

Sample at 10x per second.

Walk at 2 yard mark back and forth every 30 seconds for 3 minutes.

Synchronize stopwatch with start of test. Begin computer measurements at 15:48:00, at 15:49:00 appear in field at 2 yard mark. Walk back and forth (once time) every 30 seconds. Leave the field at 15:53:00.

Significant experiment data:

\begin{tabular}{|r|r|r|}
\hline time & sonar & IR distance \\
\hline $15: 49: 3.903$ & 540 & 461 \\
\hline $15: 49: 32.504$ & 580 & 621 \\
\hline $15: 50: 2.606$ & 520 & 540 \\
\hline $15: 50: 32.510$ & 548 & 430 \\
\hline $15: 51: 2.494$ & 532 & 606 \\
\hline $15: 51: 32.876$ & 532 & 415 \\
\hline $15: 52: 1.963$ & 520 & 221 \\
\hline
\end{tabular}

Average of sonar significant readings at 2 yards: 538.86

Average of IR distance significant readings at 2 yards: 470.57

The sonar sensor has a wider field of vision and the subject is in range for a couple seconds. The IR distance sensor has a narrow focus beam and the subject may only make one significant reading (sampled at 10x per second) as they walk by. 
Experiment 8 2, July, 2010

Sensors: Phidgets sonar (0) and IR distance (1)

Field: Top of Spanagel Hall, sensors on 0 yard mark, marks measured out every 1 yard away to 6 yds.

Purpose: Test accuracy of distance measurements

Conditions: wind $9 \mathrm{mph}, 61 \mathrm{deg}$. F.

Program: java Sensorfilter 1052

Implementation:

Synchronize stopwatch with start of test. Begin computer measurements at 15:59:00, at 16:00:00 appear in field at 1 yard mark in front of sensors and stand in place. Every 30 seconds move 1 yard back. Continue out to 6 yard mark. Leave the field after standing at the 6 yard mark for 30 seconds. End recording at 16:04:00.

\begin{tabular}{|l|r|r|r|r|}
\hline & ave sonar & ave IR & mode sonar & mode IR \\
\hline start & 2018.7 & 20.61 & 2018 & 22 \\
\hline 1 yard & 365.02 & 484.04 & 264 & 509 \\
\hline 2 yds & 541.35 & 298.86 & 532 & 289 \\
\hline 3 yds & 1160.84 & 109.2 & 856 & 21 \\
\hline 4 yds & 1660.49 & 24.38 & 2018 & 21 \\
\hline 5 yds & 1867.97 & 20.18 & 2018 & 21 \\
\hline 6 yds & 2016.06 & 20.09 & 2018 & 21 \\
\hline leave & 2018.29 & 20.12 & 2018 & 21 \\
\hline
\end{tabular}

The mode would suggest the sonar sensor can see to 3 yards and the IR distance can see 2 yards. However, the averages suggest they can see further. The sonar sensor starts high and goes low; a reading around 2000 occurs when nothing is in range. The IR distance sensor starts low and goes high; a reading around 20 occurs when nothing is in range.

The sonar sensor shows average raw values of 1867 at 5 yards, which indicates it detects the subject, and 2016 at 6 yards which indicates that it does not detect anything. The IR distance sensor shows an average raw value of 856 at 3 yards, and an average value of 2018 at 4 yards which is the same as when it sees to infinity. We may interpret these as a maximum range of 5 yards for sonar and 3 yards for IR distance. 
Experiment 9 2, July, 2010

Sensors: Phidgets sonar (0) and IR distance (1)

Field: Top of Spanagel Hall, sensors on 0 yard mark, marks measured out every 1 yard away to 10 yds.

Purpose: Test accuracy of distance measurements

Conditions: Wind $9 \mathrm{mph}$, temp. 61 deg. F.

Program: java Sensorfilter 1052

Implementation:

Reverse of previous test, this time starting at 6 yards and moving 1 yard closer to sensors every 30 seconds.

Synchronize stopwatch with start of test. Begin computer measurements at 16:16:00, at 16:17:00 appear in field at 6 yards, directly in front of sensors. Every 30 seconds move 1 yard closer to sensors. Leave the field after standing at the 1 yard mark for 30 seconds. End recording at 16:27:00.

It should be noted that there was a person walking in the experiment field about 15 yards away, but analysis of data shows this had no impact on the sensors as they can not see a person at that distance.

\begin{tabular}{|l|r|r|}
\hline & ave sonar & ave IR dist \\
\hline start & 2018.85 & 22.51 \\
\hline 6 yds & 2015.88 & 22.44 \\
\hline 5 yds & 1806.93 & 22.52 \\
\hline 4 yds & 1522.21 & 28.61 \\
\hline 3 yds & 986.023 & 99.89 \\
\hline 2 yds & 556.54 & 143.16 \\
\hline 1 yd & 279.66 & 514.32 \\
\hline leave & 1990.16 & 27.49 \\
\hline
\end{tabular}

The data indicates the sonar sensor has a range of 5 yards and the IR distance can see to 3 yards, when the subject is standing stationary in range of the sensor. 
Experiment 10 2, July, 2010

Sensors: Phidgets sonar (0) and IR distance (1)

Field: Top of Spanagel Hall, sensors on 0 yard mark, marks measured out every 1 yard away to 6 yds.

Purpose: Test accuracy of distance measurements

Conditions: wind $8 \mathrm{mph}$, temp. $63 \mathrm{deg}$. F.

Program: java Sensorfilter 1052

Implementation:

Start at 6 yards, walk 1 yard toward sensors every 30 seconds.

Synchronize stopwatch with start of test. Begin computer measurements at 16:23:00, at 16:24:00 appear in field at 6 yards, directly in front of sensors. Every 30 seconds move 1 yard closer to sensors. Leave the field after standing at the 1 yard mark for 30 seconds. End recording at 16:27:00.

Sonar Analysis:

6 yards away, average reading 2018 (doesn't see me)

5 yards, we may infer the sensor is beginning to detect the subject. Raw sensor reading averages 1735 , which equates to $423 \mathrm{~cm}, 13.8$ feet. The sensor measurement indicates an object at 4.6 yards, yet the data is noisy. Sometimes it says 2022, other times it dips down to 1429 .

4 yards, average reading 1141, which equates to $278 \mathrm{~cm}$. 9.1 feet, approx 3 yards.

3 yards, average reading 864 , which equates to $210 \mathrm{~cm}$. 6.89 feet, 2.3 yards.

2 yards, average reading 532, which equates to $129 \mathrm{~cm}$. 4.2 feet, 1.41 yards.

1 yard, average reading 256 , which equates to $62.51 \mathrm{~cm}$. 2 feet, 0.68 yards.

Discussion: The sensor is not very accurate, and the data is noisy. It does not follow the Phidgets spec of being able to see a person at 6.45 yards. As noted, it just begins to detect the subject in the field at 5 yards.

IR distance analysis:

When nothing is in view, the raw values hover around 24 or 25 .

1 yard away: Raw value is 546 , which equates to 0.46 yards

2 yards away: Raw value is 273 , which equates to 1.12 yards

3 yards away: Raw value is 161

4 yards away: Raw value is 25 (nothing)

This result is very promising. The product spec says that it is meant to measure distances accurately out to $80 \mathrm{~cm}$, however we can use it as a binary sensor (a subject is within field of vision or not) with range to 3 yards. 
Experiment 11 7, July, 2010

Sensors: Phidgets sonar (0) and IR distance (1)

Purpose: Test angle conditions 20 degrees left

Field: Top of Spanagel Hall, sensors on 0 yard mark, marks measured out every 1 yard away to 10 yds. with angle measurements.

Purpose: Test angle measurements at 20 degrees left.

Conditions: calm wind, 57 degrees F.

Program: java Sensorfilter 1052

Implementation:

Synchronize stopwatch with start of test. Begin computer measurements at 11:17:00, at 11:18:00 appear in field at 1 yards, angle 20 degrees left. Every 30 seconds move 1 yard away from sensors. Leave the field after standing at the 6 yard mark for 30 seconds. End recording at 11:21:00.

\section{Analysis:}

The sonar sensor gives a high reading around 2000 when nothing is in view and the Raw Values go lower when an object is within range. The IR distance sensor gives a small value around 20 when nothing is in range and goes higher when an object crosses it's beam.

We see the sonar sensor works fairly well at this angle however we must choose a threshold value at which to judge the results. If we go by a threshold of 1000, (meaning the sonar sensor changes reading from 2000 to some number below 1000 to indicate an object present,) the sonar can see out to 3 yards. If we lessen our threshold restrictions and say the sonar âĂIJseesâĂİ an object when a threshold of 500 is crossed, then the results become confusing. The sonar changed less than 500 units when the subject was at 4 yards-indicating it didn't really notice anything-and it changed more than 500 units with a subject at 5 yards. Thus, a smaller threshold gives us a probability that there is an object there; a larger threshold indicates with more certainty that an object has come into view. So, a threshold of 1000 lets us know with certainty that something is there, and a threshold of 500 means that we may or may not detect an object within range. With a higher threshold we trade distance for certainty. The infrared distance sensor did not notice anything at this angle. It has a rather narrow beam.

\begin{tabular}{|r|r|r|r|r|}
\hline & ave sonar & ave IR & mode sonar & mode IR \\
\hline start & 2018.38 & 18.77 & 2018 & 20 \\
\hline 1 yard & 1219.75 & 18.68 & 2018 & 20 \\
\hline 2 yds & 1609.78 & 18.73 & 2018 & 20 \\
\hline 3 yds & 1994.72 & 18.8 & 2018 & 20 \\
\hline 4 yds & 2016.32 & 18.8 & 2018 & 20 \\
\hline 5 yds & 1961.32 & 18.77 & 2018 & 20 \\
\hline 6 yds & 2018.52 & 18.95 & 2018 & 20 \\
\hline leave & 2018.58 & 19.03 & 2018 & 20 \\
\hline
\end{tabular}

\begin{tabular}{|r|r|r|r|r|r|r|}
\hline & min sonar & min IR & max sonar & max IR & std dev SON & std dev IR \\
\hline start & 2010 & 16 & 2026 & 22 & 2.53 & 1.54 \\
\hline 1 yard & 248 & 16 & 2024 & 21 & 723.83 & 1.59 \\
\hline 2 yds & 263 & 16 & 2024 & 22 & 615.27 & 1.51 \\
\hline 3 yds & 588 & 16 & 2026 & 22 & 157.65 & 1.62 \\
\hline 4 yds & 1689 & 16 & 2025 & 22 & 22.67 & 1.56 \\
\hline 5 yds & 1425 & 16 & 2024 & 21 & 153.58 & 1.61 \\
\hline 6 yds & 2010 & 16 & 2026 & 22 & 2.51 & 1.67 \\
\hline leave & 2010 & 16 & 2026 & 22 & 2.5 & 1.68 \\
\hline
\end{tabular}


Experiment 12 2, July, 2010

Sensors: Phidgets sonar (0) and IR distance (1)

Field: Top of Spanagel Hall, sensors on 0 yard mark, marks measured out every 1 yard away to 10 yds.

Purpose: Test angle measurements at 20 degrees Right

Program: java Sensorfilter 1052

Implementation:

This experiment was not done, as we focused first on left-hand-side angles and created a reasonable model from the left side alone. 
Experiment 13 7, July, 2010

Sensors: Phidgets sonar (0) and IR distance (1)

Field: Top of Spanagel Hall, sensors on 0 yard mark, marks measured out every 1 yard away to 10 yds.

Purpose: Test accuracy of sensors at 30 degrees Left.

Program: java Sensorfilter 1052

Implementation:

Synchronize stopwatch with start of test. Begin computer measurements at 11:33:00, at 11:34:00 appear in field at 1 yard, directly in front of sensors. Every 30 seconds move 1 yard away from sensors. Leave the field after standing at the 6 yard mark for 30 seconds. End data recording at 11:37:00.

\section{Analysis:}

The IR distance sensor has too narrow a focus to be useful at 30 degrees. The sonar sensor is only able to detect objects at 2 yard at this angle. There is a significant change in Raw Sensor Values between 2 yards and 3 yards; the reading at 2 yards shows the definite presence of an object and the reading at 3 yards does not.

\begin{tabular}{|r|r|r|r|r|}
\hline & ave sonar & ave IR & mode sonar & mode IR \\
\hline start & 2018.68 & 19.77 & 2018 & 21 \\
\hline 1 yard & 371.49 & 19.8 & 256 & 21 \\
\hline 2 yds & 1965.41 & 19.82 & 2018 & 21 \\
\hline 3 yds & 2018.69 & 19.76 & 2018 & 21 \\
\hline 4 yds & 2018.67 & 19.79 & 2018 & 21 \\
\hline 5 yds & 2018.42 & 19.78 & 2018 & 21 \\
\hline 6 yds & 2018.68 & 19.75 & 2018 & 21 \\
\hline leave & 2018.65 & 19.75 & 2018 & 21 \\
\hline
\end{tabular}

\begin{tabular}{|r|r|r|r|r|r|r|}
\hline & min sonar & min IR & max sonar & max IR & std dev SON & std dev IR \\
\hline start & 2011 & 16 & 2026 & 23 & 2.59 & 1.98 \\
\hline 1 yard & 252 & 16 & 2021 & 21 & 421.47 & 1.91 \\
\hline 2 yds & 256 & 16 & 2026 & 23 & 294.33 & 1.93 \\
\hline 3 yds & 2010 & 16 & 2026 & 23 & 2.61 & 1.95 \\
\hline 4 yds & 2011 & 16 & 2026 & 23 & 2.54 & 1.95 \\
\hline 5 yds & 2010 & 16 & 2025 & 22 & 2.71 & 1.95 \\
\hline 6 yds & 2010 & 16 & 2026 & 23 & 2.74 & 1.95 \\
\hline leave & 2010 & 16 & 2026 & 23 & 2.62 & 1.95 \\
\hline
\end{tabular}


Experiment 14 7, July, 2010

Sensors: Phidgets sonar (0) and IR distance (1)

Field: Top of Spanagel Hall, sensors on 0 yard mark, marks measured out every 1 yard away to 10 yds.

Purpose: Test angle measurements at 30 degrees Right

Program: java Sensorfilter 1052

Implementation:

This experiment was not done, as we focused first on left-hand-side angles and created a reasonable model from the left side alone. 
Experiment 15 7, July, 2010

Sensors: Phidgets IR motion sensors, $[0,1,2]$

Field: Top of Spanagel Hall, sensors on 0 yard mark, marks measured out every 1 yard away to 10 yds.

Purpose: Test accuracy of distance measurements

Program: java Sensorfilter 1053

Implementation:

Walk at 1 yard intervals. Sensor makes 10 samples per second. The product spec sheet says the sensor is rated to 5 meters; we are testing this claim. Three sensors are taped down on a board, laid on the ground.

Synchronize stopwatch with start of test. Begin computer measurements at 12:02:00, at 12:03:00 appear in field at 1 yards, walk directly past sensors perpendicular to their location (across their field of view.) The distance traveled is approximately 10 yards. Stop and remain motionless after reaching edge of test field. Then every 30 seconds move 1 yard further away from sensors and walk past their field of vision again. Leave the field after walking past the 7 yard mark. End recording at 12:07:00.

\section{Analysis:}

There are two ways to go about acknowledging detection: We can think in terms of readings occurring that are greater than 2 standard deviations from the recent average, or we can think of Raw Sensor Values crossing a threshold. If we go by the 2 standard deviations, the sensors work well out to 3 meters, and then their success at detection becomes a probability measurement. At four yards, 2 of 3 detected the moving human subject and at 5 yards 1 of 3 sensors detected the moving subject. It may be better to go by a threshold value of somewhere between 500 and 1000 .

\begin{tabular}{|r|r|r|r|r|r|r|}
\hline & ave 0 & ave 1 & ave 2 & min 0 & min 1 & $\min 2$ \\
\hline start & 2001.26 & 2080.63 & 2076.36 & 1673 & 1313 & 1745 \\
\hline 1 yard & 2007.72 & 2075.72 & 2073.94 & 8 & 12 & 218 \\
\hline 2 yds & 1995.94 & 2081.6 & 2073.59 & 1333 & 1624 & 1513 \\
\hline 3 yds & 1985.11 & 2092.34 & 2071.13 & 1609 & 1841 & 1885 \\
\hline 4 yds & 2014.42 & 2064.44 & 2077.46 & 1702 & 1773 & 1929 \\
\hline 5 yds & 1993.96 & 2087.5 & 2076.47 & 1725 & 1850 & 1945 \\
\hline 6 yds & 1996.00 & 2076.75 & 2074.72 & 1833 & 1873 & 1990 \\
\hline 7 yds & 1998.38 & 2082.95 & 2072.81 & 1741 & 1842 & 1901 \\
\hline
\end{tabular}

\begin{tabular}{|r|r|r|r|r|r|r|}
\hline & $\max 0$ & $\max 1$ & $\max 2$ & stddev 0 & stddev 1 & stddev 2 \\
\hline start & 2414 & 2430 & 2283 & 124.06 & 122.34 & 68.5 \\
\hline 1 yard & 4040 & 3772 & 2719 & 391.74 & 305.45 & 185.11 \\
\hline 2 yds & 2858 & 2790 & 2686 & 124.19 & 116.2 & 100.00 \\
\hline 3 yds & 2525 & 2510 & 2378 & 124.98 & 104.84 & 68.71 \\
\hline 4 yds & 2557 & 2290 & 2354 & 127.93 & 100.03 & 61.13 \\
\hline 5 yds & 2306 & 2346 & 2198 & 117.57 & 108.91 & 49.63 \\
\hline 6 yds & 2182 & 2246 & 2206 & 78.53 & 76.16 & 41.21 \\
\hline 7 yds & 2366 & 2319 & 2246 & 111.51 & 100.02 & 53.25 \\
\hline
\end{tabular}

The following chart for Exp. 15 assumes we are using 2 standard deviations as our detection method. In this case detection is based on min or max reading being beyond 2 standard deviations. 


\begin{tabular}{|r|r|}
\hline & all detect? \\
\hline start & na \\
\hline 1 yard & yes \\
\hline $2 \mathrm{yds}$ & yes \\
\hline $3 \mathrm{yds}$ & 1 and 2 only \\
\hline $4 \mathrm{yds}$ & 1 only \\
\hline $5 \mathrm{yds}$ & no \\
\hline $6 \mathrm{yds}$ & no \\
\hline $7 \mathrm{yds}$ & no \\
\hline
\end{tabular}

If we use a threshold value of 500 and examine both the minimum and maximum Raw Sensor Values then all of the sensors can detect a subject walking across the field of vision at 3 yards, but not further. An increase in threshold values provides an increase in accuracy with a decrease in distance. Lowering our threshold increases our distance of possible detection and also increases our chances of false positives. 
Experiment 16 8, July, 2010

Sensors: Three Phidgets 1111 IR Motion sensors

Field: Circular course in large parking lot at Fort Ord. There were concentric rings (like a target) drawn in chalk 1 yard apart. The circles were drawn out to 7 yards from the center.

Weather: Sunny, no clouds, slight wind.

Purpose: Test accuracy of distance measurements

Program: java Sensortest 1093

Implementation:

Synchronize stopwatch with start of test. Begin computer measurements at 14:29:00, at 11:30:00 appear in field at 1 yards. Every 60 seconds walk in a complete circle and then move 1 yard closer to sensors. End recording at 11:34:00.

A threshold of 500 points is explored. The last column shows what the sensor would report if we chose a threshold of 500 points to indicate detection of a subject:

\begin{tabular}{|r|r|r|r|r|r|r|}
\hline & ave 0 & ave 1 & ave 2 & stddev 0 & stddev 1 & stddev 2 \\
\hline start & 1997.79 & 2081.03 & 2073.54 & 181.02 & 294.84 & 165.36 \\
\hline 1 yard & 2003.96 & 2087.2 & 2086.94 & 419.11 & 405.08 & 362.41 \\
\hline 2 yds & 1997.58 & 2076.73 & 2067.53 & 173.58 & 225.23 & 105.31 \\
\hline 3 yds & 1997.79 & 2092.94 & 2080.65 & 163.00 & 187.59 & 85.29 \\
\hline 4 yds & 1997.68 & 2070.25 & 2074.19 & 158.03 & 241.04 & 92.78 \\
\hline 5 yds & 1993.8 & 2100.18 & 2075.15 & 244.17 & 319.28 & 91.86 \\
\hline 6 yds & 1978.4 & 2072.19 & 2071.29 & 211.52 & 273.94 & 111.17 \\
\hline 7 yds & 2010.37 & 2071.73 & 2077.94 & 202.72 & 246.84 & 97.78 \\
\hline
\end{tabular}

\begin{tabular}{|r|r|r|r|r|r|r|r|}
\hline & $\min 0$ & $\min 1$ & $\min 2$ & $\max 0$ & $\max 1$ & $\max 2$ & all detect? (threshold 500) \\
\hline start & 1157 & 1021 & 680 & 3391 & 3814 & 3475 & na \\
\hline 1 yard & 12 & 12 & 10 & 4020 & 4081 & 4024 & yes \\
\hline 2 yds & 1249 & 975 & 1441 & 3154 & 3391 & 2808 & yes \\
\hline 3 yds & 1453 & 1285 & 1693 & 2414 & 2598 & 2402 & 0 and 1 only \\
\hline 4 yds & 1649 & 1601 & 1861 & 2345 & 2770 & 2310 & 1 only \\
\hline 5 yds & 1541 & 1253 & 1864 & 2650 & 2738 & 2290 & 0 and 1 only \\
\hline 6 yds & 1633 & 1245 & 1761 & 2674 & 2606 & 2314 & 0 and 1 only \\
\hline 7 yds & 1374 & 1489 & 1829 & 2434 & 2870 & 2354 & and 1 only \\
\hline
\end{tabular}

False positives: There are times when one sensor shows high readings 500 points above average, yet the other two sensors do not. The sensors were taped to a wooden board in a line, about 4 inches apart and the board was placed on the ground. The board-and thus the sensors-may be moving in the breeze, or may be detecting their own wires moving because they are not taped down. The reading above the threshold would indicate that an object is present, yet the subject was not moving at the time. For example the middle sensor showed a value of 2870 when the subject was standing at 7 yards. Previous tests have shown that the sensors can not see 7 yards, and at the time in question, the subject was also standing still. We know from previous experimentation that the sensors can not detect a stationary subject.

\section{$14: 33: 24.831278624804826178628702122$}

In order to actually detect a subject, we may need to filter out singular spikes and dips and instead only count clusters of high points occurring together. One spike for $1 / 10$ th of a second does not necessarily mean that somebody jumped into view and then quickly left. 
Experiment 17 2, July, 2010

Sensors: Phidgets sonar (0) and IR distance (1)

Field: Top of Spanagel Hall, sensors on 0 yard mark, marks measured out every 1 yard away to 10 yds.

Purpose: 10 degree angle test, part of a series of angle test to determine the sensors' field of vision Program: java Sensorfilter 1052

Implementation:

Synchronize stopwatch with start of test. Begin computer measurements at 16:23:00, at 16:24:00 appear in field at 6 yards, directly in front of sensors. Every 30 seconds move 1 yard closer to sensors. Leave the field after standing at the 1 yard mark for 30 seconds. End recording at 16:27:00.

\begin{tabular}{|r|r|r|r|r|}
\hline & ave sonar & ave IR & mode sonar & mode IR \\
\hline start & 2018.71 & 18.65 & 2018 & 19 \\
\hline 1 yard & 352.49 & 801.71 & 264 & 937 \\
\hline 2 yds & 573.29 & 43.57 & 580 & 19 \\
\hline 3 yds & 1089.91 & 18.76 & 848 & 19 \\
\hline 4 yds & 1726.19 & 18.68 & 2018 & 20 \\
\hline 5 yds & 1889.5 & 18.84 & 2018 & 20 \\
\hline 6 yds & 1989.45 & 18.79 & 2018 & 20 \\
\hline leave & 2018.39 & 18.89 & 2018 & 20 \\
\hline
\end{tabular}

\begin{tabular}{|r|r|r|r|r|r|r|}
\hline & min sonar & min IR & max sonar & max IR & std dev sonar & std dev IR dist \\
\hline start & 2011 & 16 & 2026 & 22 & 2.61 & 1.5 \\
\hline 1 yard & 256 & 16 & 2023 & 1167 & 382.43 & 246.15 \\
\hline 2 yds & 260 & 16 & 1667 & 1092 & 119.83 & 138.23 \\
\hline 3 yds & 580 & 16 & 2016 & 92 & 350.94 & 4.48 \\
\hline 4 yds & 856 & 16 & 2023 & 21 & 286.51 & 1.54 \\
\hline 5 yds & 1126 & 16 & 2025 & 21 & 185.62 & 1.61 \\
\hline 6 yds & 1437 & 16 & 2025 & 21 & 78.03 & 1.6 \\
\hline leave & 1917 & 16 & 2026 & 22 & 4.89 & 1.63 \\
\hline
\end{tabular}

Analysis: There are several ways to analyze the data. The first is to use a threshold value based on standard deviation. The standard deviation for the period when nothing was in view is very small, 2.61 for the sonar sensor and 1.5 for the IR distance sensor. Using a standard deviation of 2.61 we can suggest a threshold of 100 points is well over 2 standard deviations and may be used for detection. At 5 yards the average sonar reading was 1889.5 and this is just over the 100 point threshold, indicating that the sensor detected the subject. 5 yards corresponds with the manufacturer spec sheet. A threshold of 100 raw data values for the IR distance sensor may also be chosen, and we can determine the sensor can see 1 yard. The average IR distance reading at 1 yard is 801.71 , which is more than 100 points over the value of 21 when nothing is in the field. It can be deduced that the IR distance sensor can only see 1 yard at the 10 degree angle.

A second method can be used where the trend in standard deviation over a series of distances. The graph shows that the standard deviation is higher than 100 points for the sonar sensor from 1 to 5 yards. The IR distance sensor has a similar bubble where standard deviation is over 100 points from 1 to 2 yards. We may hypothesize that examining the standard deviation and choosing a threshold of 100 points will indicate the sensors see a subject within range.

A third method is to examine the mode over a period of time. The raw data value when the sonar sensor sees nothing is around 2018 and for the IR distance sensor it is 21 . During any period where the subject was stationary and standing within range of the sensor, we can say the sensor 
detects a subject if the mode is any value other than the value the sensor shows when looking to infinity. For example, any value significantly different than 2018 for the sonar sensor and 21 for the IR distance sensor indicates someone is within the sensors' field of vision. This method gives conservative results for sensor vision: The sonar sensor can see 3 yards (not 5) and the IR distance sensor can see 1 yard away at 10 degrees.

Choosing the first method-using thresholds for raw sensor values-is the most appropriate, since data will be interpreted as it comes in. We may not expect a subject to stand still for 30 seconds in the field as we did for this test; therefore, it would be difficult to take the mode or the standard deviation over a long time period, such as 30 seconds. If a time period is used to examine data, it should be short, such as a few seconds at a time. This data can be filtered with a noise filtering algorithm and a detection decision can be made if raw data values exceed a threshold for that period of time.

Using the first method: A 100 point threshold for both sonar and IR distance shows that the sonar sensor can see 5 yards at 10 degrees, and the IR distance sensor can see 1 yard at 10 degrees. 
Experiment 18 2, July, 2010

Sensors: Phidgets sonar (0) and IR distance (1)

Field: Top of Spanagel Hall, sensors on 0 yard mark, marks measured out every 1 yard away to 10 yds.

Purpose: Test accuracy of distance measurements

Program: java Sensorfilter 1052

Implementation:

Synchronize stopwatch with start of test. Begin computer measurements at 16:23:00, at 16:24:00 appear in field at 6 yards, directly in front of sensors. Stand still in front of sensors. Every 30 seconds move 1 yard closer to sensors. Leave the field after standing at the 1 yard mark for 30 seconds. End recording at 16:27:00.

\begin{tabular}{|r|r|r|r|r|}
\hline & ave sonar & ave IR & mode sonar & mode IR \\
\hline start & 2018.58 & 21.78 & 2018 & 23 \\
\hline 1 yard & 1945.65 & 21.46 & 2018 & 23 \\
\hline 2 yds & 2016.86 & 21.48 & 2018 & 23 \\
\hline 3 yds & 2018.54 & 21.67 & 2018 & 23 \\
\hline 4 yds & 2018.73 & 21.56 & 2018 & 23 \\
\hline 5 yds & 2018.69 & 21.77 & 2018 & 23 \\
\hline 6 yds & 2018.39 & 21.29 & 2018 & 23 \\
\hline leave & 2018.47 & 21.29 & 2018 & 22 \\
\hline
\end{tabular}

\begin{tabular}{|r|r|r|r|r|r|r|}
\hline & min sonar & min IR dist & max sonar & max IR dist & stdev sonar & stdev IR dist \\
\hline start & 2011 & 16 & 2026 & 82 & 2.58 & 4.82 \\
\hline 1 yard & 288 & 16 & 2026 & 33 & 313 & 2.48 \\
\hline 2 yds & 1621 & 16 & 2026 & 41 & 23.85 & 2.76 \\
\hline 3 yds & 2012 & 16 & 2025 & 97 & 2.52 & 5.06 \\
\hline 4 yds & 2013 & 16 & 2026 & 41 & 2.60 & 2.64 \\
\hline 5 yds & 2010 & 16 & 2025 & 52 & 2.67 & 3.34 \\
\hline 6 yds & 2010 & 16 & 2025 & 24 & 2.52 & 2.37 \\
\hline leave & 2010 & 16 & 2026 & 42 & 2.52 & 2.55 \\
\hline
\end{tabular}

Analysis: When the field was empty at the start of the test, the standard deviation for sonar and IR distance sensors were 2.58 and 4.82 respectively. Choosing a 100 point threshold for both sensors ensures that values taken from average readings are well over two standard deviations. Looking at the average reading, only the sonar sensor is able to detect a subject; at 1 yard the sonar sensor went from 2011 (nothing in view) to 288. This is a significant move in raw data values. The sonar sensor shows average values at 1621 when the subject is at 2 yards. Again, this is over the threshold, however it is much closer to the 2011 reading when the sensor sees to infinity. This threshold shows the sonar sensor can see a subject at 2 yards when angled at 40 degrees. If a threshold of 500 points is chosen, that range is reduced to 1 yard at 40 degrees. The IR distance averages showed regularly in the under-100 range and never crossed a 100 point threshold from average, during the entire test. It can be concluded that the IR distance sensor is unable to detect a subject at 40 degrees from center. 
Experiment 19 2, July, 2010

Sensors: Phidgets 1111 IR Motion sensors

Field: Circular course in large parking lot at Fort Ord. There were concentric rings (like a target) drawn in chalk 1 yard apart. The circles were drawn out to 7 yards from the center.

Weather: Sunny, no clouds, slight wind.

Purpose: Test accuracy of distance measurements

Program: java Sensortest 1093

Implementation:

Synchronize stopwatch with start of test. Begin computer measurements at 11:46:00, at 11:47:00 appear in field at 7 yards. Every 60 seconds walk in a complete circle and then move 1 yard closer to sensors. Stop and wait for 60 second mark to occur again, then repeat. End recording at 11:56:00.

\begin{tabular}{|c|c|c|c|c|c|c|}
\hline & ave 0 & ave 1 & ave 2 & stddev 0 & stddev 1 & stddev 2 \\
\hline start & 2061.55 & 1948.62 & 2054.21 & 138.61 & 241.07 & 163.06 \\
\hline $7 \mathrm{yds}$ & 2063.65 & 1958.97 & 2057.39 & 87.23 & 178.21 & 87.15 \\
\hline $6 \mathrm{yds}$ & 2064.44 & 1955.56 & 2053.21 & 133.51 & 173.57 & 101.24 \\
\hline $5 \mathrm{yds}$ & 2064.4 & 1969.55 & 2057.6 & 96.82 & 168.97 & 108.07 \\
\hline $4 \mathrm{yds}$ & 2064.75 & 1960.64 & 2061.41 & 120.72 & 187.94 & 142.14 \\
\hline $3 \mathrm{yds}$ & 2064.34 & 1955.32 & 2058.28 & 195.98 & 265.86 & 224.93 \\
\hline $2 \mathrm{yds}$ & 2065.3 & 1962.95 & 2059.11 & 329.65 & 313.59 & 323.83 \\
\hline 1 yard & 2070.96 & 1972.66 & 2059.44 & 465.32 & 493.12 & 537.73 \\
\hline last $60 \mathrm{sec}$ & 2064.26 & 1956.69 & 2055.58 & 139.70 & 211.35 & 188.88 \\
\hline
\end{tabular}

\begin{tabular}{|r|r|r|r|r|r|r|c|}
\hline & $\min 0$ & $\min 1$ & $\min 2$ & $\max 0$ & $\max 1$ & $\max 2$ & all detect (threshold 1000)? \\
\hline start & 909 & 1062 & 873 & 2626 & 2698 & 2662 & na \\
\hline 7 yds & 1845 & 1625 & 1853 & 2318 & 2461 & 2342 & no \\
\hline 6 yds & 1873 & 1525 & 1737 & 2294 & 2394 & 2302 & no \\
\hline 5 yds & 1707 & 1541 & 1709 & 2338 & 2378 & 2325 & no \\
\hline 4 yds & 1549 & 1345 & 1481 & 2590 & 2478 & 2562 & yes \\
\hline 3 yds & 1025 & 944 & 906 & 3031 & 2899 & 3091 & yes \\
\hline 2 yds & 12 & 12 & 16 & 4008 & 3891 & 3735 & yes \\
\hline 1 yds & 11 & 8 & 9 & 4083 & 4083 & 4083 & \\
\hline last 60 sec & 852 & 1389 & 848 & 2766 & 2430 & 3435 & \\
\hline
\end{tabular}

Analysis: When a threshold of 1000 is chosen, all three sensors perform consistently and detect a subject moving across their field of view at 3 yards. A threshold of 500 would allow each sensor to detect a subject at 4 yards. 
Experiment 20 14, July, 2010

Sensors: Phidgets sonar (0) and IR distance (1)

Field: Circular course in large parking lot at Fort Ord. There were concentric rings (like a target) drawn in chalk 1 yard apart. The circles were drawn out to 7 yards from the center.

Weather: Sunny, slight wind, no clouds.

Program: java Sensorfilter 1093

Notes: Shirt blowing in wind slightly. This may not be enough for the motion sensor to detect. Cars going by 65 yards away were determined to be out of range for sensors.

Implementation:

This experiment is identical to Experiment 19. Synchronize stopwatch with start of test. Begin computer measurements at 12:00:00, then at 12:01:00 appear in field at 7 yards. Every 60 seconds walk in a complete circle and then move 1 yard closer to sensors. End recording at 12:09:00.

\begin{tabular}{|r|r|r|r|r|r|r|}
\hline & ave 0 & ave 1 & ave 2 & stddev 0 & stddev 1 & stddev 2 \\
\hline start & 2059.13 & 1946.68 & 2051.99 & 125.58 & 274.17 & 141.33 \\
\hline 7 yds & 2064.81 & 1960.35 & 2059.10 & 94.57 & 230.33 & 119.01 \\
\hline 6 yds & 2067.48 & 1965.01 & 2056.64 & 166.43 & 246.36 & 88.35 \\
\hline 5 yds & 2063.45 & 1964.78 & 2057.01 & 88.43 & 203.88 & 91.89 \\
\hline 4 yds & 2066.41 & 1956.95 & 2057.66 & 136.44 & 219.99 & 139.72 \\
\hline 3 yds & 2064.50 & 1962.84 & 2058.37 & 202.48 & 237.75 & 216.59 \\
\hline 2 yds & 2062.92 & 1959.74 & 2053.85 & 321.23 & 334.30 & 347.78 \\
\hline 1 yard & 2069.38 & 1970.72 & 2064.17 & 508.59 & 467.93 & 516.11 \\
\hline last 60 sec & 2061.01 & 1945.23 & 2055.51 & 253.70 & 232.36 & 189.25 \\
\hline
\end{tabular}

\begin{tabular}{|c|c|c|c|c|c|c|c|}
\hline & $\min 0$ & $\min 1$ & $\min 2$ & $\max 0$ & $\max 1$ & $\max 2$ & all detect (threshold 1000)? \\
\hline start & 1245 & 1197 & 1153 & 2742 & 2794 & 2802 & na \\
\hline $7 \mathrm{yds}$ & 1813 & 1304 & 1721 & 2346 & 2561 & 2350 & no \\
\hline $6 \mathrm{yds}$ & 1845 & 1285 & 1769 & 2375 & 2534 & 2294 & no \\
\hline $5 \mathrm{yds}$ & 1770 & 1301 & 1741 & 2346 & 2458 & 2357 & no \\
\hline $4 \mathrm{yds}$ & 1538 & 1409 & 1505 & 2650 & 2506 & 2500 & no \\
\hline $3 \mathrm{yds}$ & 1077 & 956 & 832 & 3171 & 2710 & 2946 & yes \\
\hline $2 \mathrm{yds}$ & 44 & 264 & 85 & 3675 & 3331 & 3777 & yes \\
\hline 1 yard & $\overline{12}$ & 8 & 11 & 4083 & 4082 & 4083 & yes \\
\hline last $60 \mathrm{sec}$ & $\overline{12}$ & 900 & 178 & 3807 & 2667 & 2991 & \\
\hline
\end{tabular}

Analysis: Using a threshold of 1000 ensures that the subject is indeed detected with little chance of false positives. This threshold corresponds to the hypothesis that a true positive (as opposed to false positive) will be greater than two standard deviations from the arithmetic mean. The threshold could be lowered to 500 in which case the sensors have a usable range to 4 yards. At this distance all the sensors report either a value 500 points above or below the arithmetic mean when the subject is moving within range. The closer the threshold value comes to the arithmetic mean, the greater the chance for false positives. 
Experiment 21 14, July, 2010

Sensors: Phidgets light sensors

Field: In office, testing the light sensors near different colored objects and in different areas of the room. Light sensor was bare with no filters or covering.

Purpose: Test indoor measurements of light sensor

Program: java Sensorfilter 1081

Implementation, times and readings:

\begin{tabular}{|l|r|r|}
\hline 1. on floor, middle of room & $14: 53: 00$ & 1072 \\
\hline 2. next to door & $14: 53: 30$ & 916 \\
\hline 3. corner A & $14: 54: 00$ & 653 \\
\hline 4. corner B & $14: 54: 30$ & 636 \\
\hline 5. corner C & $14: 55: 00$ & 613 \\
\hline 6. under table & $14: 55: 30$ & 162 \\
\hline 7. next to black shirt & $14: 56: 00$ & 625 \\
\hline 8. next to green shirt & $14: 56: 30$ & 853 \\
\hline
\end{tabular}

Using the program Rowpicker.java to determine mean values at each location:

Position 1: On floor, middle of room

The file had 149 lines.

The min value is: 1069 at time: $15: 1: 15.161$

The max value is: 1077 at time: 15:1:24.406

The arithmetic mean is: 1072.07

The standard deviation is: 2.1874798840773924

Position 2: Next to door

The file had 149 lines.

The min value is: 912 at time: $15: 1: 45.97$

The max value is: 920 at time: $15: 1: 47.407$

The arithmetic mean is: 915.85

The standard deviation is: 1.313784763728593

Position 3: Corner A

The file had 150 lines.

The min value is: 648 at time: $15: 2: 15.134$

The max value is: 656 at time: $15: 2: 18.45$

The arithmetic mean is: 653.01

The standard deviation is: 1.7359116514802702

Position 4: Corner B

The file had 150 lines.

The min value is: 632 at time: $15: 2: 58.987$

The max value is: 640 at time: $15: 2: 45.141$

The arithmetic mean is: 635.84

The standard deviation is: 0.819887044110755

Position 5: Corner C

The file had 150 lines.

The min value is: 612 at time: $15: 3: 15.47$

The max value is: 616 at time: $15: 3: 15.649$ 
The arithmetic mean is: 613.81

The standard deviation is: 1.8405846021990835

Position 6: Under table

The file had 150 lines.

The min value is: 52 at time: $15: 3: 59.987$

The max value is: 168 at time: 15:3:45.47

The arithmetic mean is: 161.89

The standard deviation is: 17.04850462847443

Position 7: Next to black shirt

The file had 150 lines.

The min value is: 540 at time: 15:4:20.45

The max value is: 712 at time: $15: 4: 24.155$

The arithmetic mean is: 624.71

The standard deviation is: 51.83820175385936

Position 8: Next to green shirt

The file had 150 lines.

The min value is: 784 at time: 15:4:45.909

The max value is: 900 at time: 15:4:51.725

The arithmetic mean is: 853.19

The standard deviation is: 33.72159754429789 
Experiment 22 14, July, 2010

Sensors: Phidgets 1127 Precision Light Sensor

Field: Quad area of Glasgow Hall.

Weather: Sunny, 17:12 PM, 68 deg. F.

Purpose: Test raw sensor data outside with filter material.

Program: java Sensortest 411

Implementation:

A local glass shop provided 5 squares of $33 \%$ gray smoked glass, (the glass blocked $66 \%$ of light,) and a cardboard light-box was constructed around the sensor. Five layers of glass were stacked above the sensor and the sensor was tested outside in the sun.

One minute test:

17:12:00 5 filters, sunlight exposure

17:12:30 5 filters, hand makes shadow over sensor, approximately 6âĂI high

Control:

uncovered sensor, Raw Value mean is 4079 . This is the peak value for the sensor. It is being over saturated with light.

17:12:00 to $17: 12: 29:$

open exposure to sun with 5 layers tinted glass gives mean Raw Value of 3059 .

17:12:30 to $17: 12: 59$

sensor in shadow with 5 layers tinted glass gives mean Raw Value 200

\section{Conclusions:}

The sensor can not function in daylight outside. It requires several layers of filter material in order to bring the light exposure down to a range it can deal with. We find that using 5 layers of $33 \%$ filter puts the sensor in a functional range outdoors and that it can be used to detect the shadow of a passing object. Light transmission with 5 filters at $33 \%$ is $0.391 \%$. $(.33 \times .33 \times .33 \times .33 \times$ .33). The Phidgets 1127 Precision Light Sensor is meant for indoor use. Most of these sensors are designed for home robotics kits, so it makes sense that they would function better indoors. 
Experiment 23 15, July, 2010

Sensors: Phidgets Precision Light Sensor 1127

Program: java Sensortest 1042

Unfiltered data, 10 samples per sec, 4 minute test, 2 sensors

Purpose: Experiment 23, Light test - WITHOUT SHADOW

Location: hallway by E120, Glasgow Hall East, NPS

Date: 15 July, 2010

Situation: 3:53 PM, sunny day outside. Hallway has banks of fluorescent lights spaced 6 feet apart.

Each bank holds one 18W U-light.

Method: Take 30 second samples in 5 different positions in the hall. Due to the time needed to move from position to position, we cut off the first $15 \mathrm{sec}$. of each sample to be sure the sensor was in the right place and taking an accurate reading. The people creating the experiment were careful not to cast a shadow over the sensors and stood well away.

NOTE: Readings are RawSensorValue. To convert to lux, the following formula is used: lux = RawSensorValue/4.095

Positions: Five positions on floor. Each location was numbered, with \#5 being at the end of the hall by the classroom E117. Positions were 5 feet apart, with number 1 between E108 and E109.

Position 1: placed directly on the floor

Uncovered:

The file had 149 lines.

The min value is: 660 at time: $15: 53: 15.272$

The max value is: 668 at time: $15: 53: 16.377$

The arithmetic mean is: 662.34

The standard deviation is: 2.2916186890964636

Covered:

The file had 149 lines.

The min value is: 192 at time: $15: 53: 15.71$

The max value is: 196 at time: 15:53:21.603

The arithmetic mean is: 192.12

The standard deviation is: 0.5684592737795264

Position 2: placed directly on the floor

Uncovered:

The file had 150 lines.

The min value is: 364 at time: $15: 53: 45.811$

The max value is: 604 at time: 15:53:51.434

The arithmetic mean is: 572.77

The standard deviation is: 65.45618417674447

Covered:

The file had 150 lines.

The min value is: 152 at time: $15: 53: 46.614$

The max value is: 168 at time: $15: 53: 47.216$

The arithmetic mean is: 166.88 
The standard deviation is: 2.9353663892138226

Position 3: placed directly on the floor

Uncovered:

The file had 150 lines.

The min value is: 636 at time: $15: 54: 15.26$

The max value is: 780 at time: $15: 54: 21.951$

The arithmetic mean is: 744.01

The standard deviation is: 30.8813484900475

Covered:

The file had 150 lines.

The min value is: 192 at time: 15:54:15.26

The max value is: 224 at time: 15:54:16.131

The arithmetic mean is: 222.16

The standard deviation is: 5.668800756193721

Position 4: placed directly on the floor

Uncovered:

The file had 150 lines.

The min value is: 536 at time: $15: 54: 45.27$

The max value is: 540 at time: $15: 54: 45.829$

The arithmetic mean is: 536.12

The standard deviation is: 0.5424280486105636

Covered:

The file had 150 lines.

The min value is: 152 at time: $15: 54: 45.27$

The max value is: 156 at time: 15:54:47.234

The arithmetic mean is: 152.39

The standard deviation is: 0.9686183337672625

Position 5: placed directly on the floor

Uncovered:

The file had 160 lines.

The min value is: 304 at time: $15: 55: 15.426$

The max value is: 324 at time: $15: 55: 22.657$

The arithmetic mean is: 320.79

The standard deviation is: 4.704324251863028

Covered:

The file had 160 lines.

The min value is: 80 at time: $15: 55: 15.24$

The max value is: 84 at time: $15: 55: 22.958$

The arithmetic mean is: 80.94

The standard deviation is: 1.6124047434201425

Conclusions: 
The light sensors are best suited for indoor use, and are able to detect variations in light intensity at different points in a hallway lit by fluorescent tubes. One filter allowing $33 \%$ light transmission was acceptable for indoor use. More filters would likely block out all indoor light. 
Experiment 24 2, July, 2010

Sensors: Phidgets Precision Light Sensor, Model 1127

Purpose: Experiment 24, Light test - SHADOW

Location: hallway by E120, Glasgow Hall East, NPS

Date: 15 July, 2010

Situation: 4:03 PM, sunny day outside. Hallway has banks of fluorescent lights spaced 6 feet apart. Each bank holds one 18W U-light.

Method: Take 30 second samples in 5 different positions in the hall. Due to the time needed to move from position to position, we cut off the first $15 \mathrm{sec}$. of each sample to be sure the sensor was in the right place and taking an accurate reading. There was a shadow from a person standing over the sensors at each of the 5 positions.

Program: \$java Sensortest 1042

Unfiltered data, 10 samples per sec, 4 minute test, 2 sensors

NOTE: Readings are RawSensorValue. To convert to lux, the following formula is used: lux = RawSensorValue/4.095

Positions: Five positions on floor. Each location was numbered, with \#5 being at the end of the hall by the classroom E117. Positions were 5 feet apart, with number 1 between E108 and E109.

Position 1: placed directly on the floor

Uncovered:

The file had 149 lines.

The min value is: 228 at time: $16: 3: 24.834$

The max value is: 248 at time: $16: 3: 15.187$

The arithmetic mean is: 242.85

The standard deviation is: 5.34913007577565

Covered:

The file had 149 lines.

The min value is: 92 at time: $16: 3: 29.758$

The max value is: 100 at time: $16: 3: 15.86$

The arithmetic mean is: 99.44

The standard deviation is: 1.5825193735204537

Position 2: placed directly on the floor

Uncovered:

The file had 150 lines.

The min value is: 200 at time: $16: 3: 46.629$

The max value is: 244 at time: $16: 3: 56.167$

The arithmetic mean is: 213.1

The standard deviation is: 14.523644226251832

Covered:

The file had 150 lines.

The min value is: 136 at time: $16: 3: 45.23$

The max value is: 140 at time: $16: 3: 47.131$ 
The arithmetic mean is: 138.23

The standard deviation is: 1.9495223989204724

Position 3: placed directly on the floor

Uncovered:

The file had 150 lines.

The min value is: 516 at time: $16: 4: 16.642$

The max value is: 568 at time: 16:4:15.36

The arithmetic mean is: 519.95

The standard deviation is: 7.306141114608779

Covered:

The file had 150 lines.

The min value is: 160 at time: $16: 4: 15.36$

The max value is: 208 at time: $16: 4: 16.842$

The arithmetic mean is: 191.92

The standard deviation is: 7.603884560057759

Position 4: placed directly on the floor

Uncovered:

The file had 149 lines.

The min value is: 140 at time: $16: 4: 45.943$

The max value is: 148 at time: 16:4:45.40

The arithmetic mean is: 143.77

The standard deviation is: 1.1413070387747355

\section{Covered:}

The file had 149 lines.

The min value is: 64 at time: $16: 4: 45.40$

The max value is: 76 at time: 16:4:46.847

The arithmetic mean is: 72.79

The standard deviation is: 2.3888124664778503

Position 5: placed directly on the floor

Uncovered:

The file had 159 lines.

The min value is: 88 at time: $16: 5: 21.479$

The max value is: 92 at time: 16:5:15.58

The arithmetic mean is: 90.01

The standard deviation is: 1.9697007097719046

Covered:

The file had 159 lines.

The min value is: 68 at time: $16: 5: 15.58$

The max value is: 68 at time: $16: 5: 15.58$

The arithmetic mean is: 68.0

The standard deviation is: 0.0 
Experiment 25 17, July, 2010

Sensors: Phidgets 1127 Precision Light Sensors, covered and uncovered

Purpose: Experiment 25, Light test, 16 positions inside a classroom

Location: Classroom E117, Glasgow Hall East, NPS

Date: 17 July, 2010

Situation: 8:45 AM, overcast day outside. Classroom has room lighting with banks of fluorescent lights spaced 4 feet apart. Each bank holds two 4-ft tube lights.

Method: Take 60 second samples in 16 different positions in the room. The positions were measured out to six foot by six foot squares. Due to the time needed to move from position to position, we cut off the first $30 \mathrm{sec}$. of each sample to be sure the sensor was in the right place and taking accurate readings. We were careful to make sure there were no shadows from people over the sensors during testing. Light sensors were placed directly on the carpeted floor of the room. There were two light sensors used during testing. One sensor was left unfiltered and one sensor was placed in a box with a gray-glass window allowing $33 \%$ light transmission.

Program: \$java Sensortest 1042

Unfiltered data, 10 samples per sec, 4 minute test, 2 sensors

Sensors: Phidgets Precision Light Sensor, Model 1127

NOTE: Readings are RawSensorValue. To convert to lux, the following formula is used: lux = RawSensorValue/4.095

Lights: Phillips Alto Universal T8 bulbs used in room. These are long-life (20,000 hr) low mercury tubes intended for industrial use. Bulbs are 32 Watts. Design lumens for the bulbs are 1200 to 2800 . The school is using a mix of bulbs with color temperature ratings of $3500 \mathrm{k}$ and $4100 \mathrm{k}$. Fluorescent bulbs generally have two spikes in color because of Mercury; one energy spike appears in the violet $(435 \mathrm{~nm})$ and another in the green $(546 \mathrm{~nm}$.) The Phidget Precision Light Sensor has a Peak Sensitivity Wavelength @ 25C of 580nm. We believe the sensor to be a Panasonic AMS104, which has at least 0.7 relative sensitivity at $546 \mathrm{~nm}$ according to the spec sheet chart.

Positions: Sixteen positions on floor. Each location was numbered, with \#1 being at front right of the room, nearest the door to outside. Four rows were made, with four sensors per row.

Diagram:

\begin{tabular}{lllll}
\hline 16 & 15 & 14 & 13 & \\
12 & 11 & 10 & 09 & door to hall \\
08 & 07 & 06 & 05 & \\
04 & 03 & 02 & 01 &
\end{tabular}

door outside

Arithmetic Mean for each position in room, with uncovered and covered sensor. 


\begin{tabular}{|l|l|l|l|l|l|l|l|}
\hline Uncovered & & & & Covered & & & \\
\hline 714.69 & 841.12 & 776.07 & 540.76 & 195.41 & 224.06 & 200.00 & 124.00 \\
\hline 941.93 & 1200.91 & 1279.46 & 1569.73 & 250.46 & 300.66 & 350.14 & 396.11 \\
\hline 1264.03 & 1742.24 & 2111.09 & 2232.17 & 403.66 & 551.05 & 637.65 & 642.60 \\
\hline 987.39 & 1783.46 & 2181.24 & 2109.11 & 274.51 & 530.51 & 637.93 & 574.95 \\
\hline
\end{tabular}


Experiment 26 19, July, 2010

Sensors: IR motion sensor

Field: Empty parking lot at Fort Ord

Purpose: Test motion sensor with nothing around

Program: java Sensortest 1051

Implementation:

Sensor by itself for 5 minutes, nothing around. Conditions are cool, foggy, start time is 9:51 AM.

Temperature is $53 \mathrm{deg}$ F. Sensor is on top of small box, approx 5 inches high.

Analysis of data using program: Rowpicker ./exp26/phidget_raw.txt 3

This program calculates the mean and standard deviation on the raw data file with results below:

The file had 2988 lines.

The min value is: 1925 at time: 9:55:3.896

The max value is: 2183 at time: 9:53:21.708

The arithmetic mean is: 2059.26

The standard deviation is: 34.65323088419163

Characteristics for this Phidgets sensor as per the 1111 Product Manual:

This sensor detects changes in infrared radiation which occur when there is movement by a person (or object) which is different in temperature from the surroundings. As this sensor detects temperature differences, it is well suited to detecting the motion of people by their body temperature. The sensor is also characterized by a narrow sensing area.

It has a 38 degree horizontal detection cone, and the product spec sheet says it can detect a human being at 5 meters away, with an operating temperature of $-20^{\circ} \mathrm{C}$ to $+85^{\circ} \mathrm{C}$.

Experiments show that at rest, the raw values are high, around 2000. When the sensors pick up motion the raw values drop to some numbers less than 100. The lowest readings (indicating the most motion) are around 99. Also, the sensor can go high and give a reading around 3000. This also indicates a detection event. Therefore, a threshold of $+/-1000$ from the âĂIJat restâĂ measurement of 2000 is a safe bet.

Analysis: A threshold value must be selected when polling the sensors. This may be $<500$ for close objects, or $<1000$ if the user chooses. The Phidget Interface Kit can be set up to detect interrupts from the sensors, however this must also be used in conjunction with a user-programmable threshold. Otherwise the device may report many false positives. 
Experiment 27 19, July, 2010

Purpose: 0 degrees IR motion sensor distance tests

Sensors: Phidgets 1111 IR motion sensor

Field: Very large open parking lot at Fort Ord base, circular course measured out every 1 yard to 7 yards.

Weather: 55 deg. F. Wind $9 \mathrm{mph}$. Cool, foggy.

Purpose: Test detection capabilities of IR Motion sensors at four compass points. Four tests are conducted, 0, 90, 180 and 270 degrees.

Program: java Sensortest 1052

Implementation:

A set of circular lines was measured and carefully drawn out in heavy chalk on the parking lot surface. With a sensor placement in the center, 7 circles were drawn out. Each circle is 1 yard further out from the center than the previous.

Sensor placed on small box, approximately 5 inches high. Start 17 yards away, (course distance plus 10 yards.) Walk up to a specific yard line on the course and then leave. Do this for each minute of the test. Begin by walking to 7 yard circle and retreating. Continue walking 1 yard closer each time, until the 1 yard line.

Synchronize stopwatch with start of test. Begin computer measurements at 10:05:00, at 10:06:00 appear in field and approach the sensor, stopping and turning around at the 7 yard mark. This test is 0 degrees to the sensors, on a compass. Every 60 seconds walk toward the sensor and stop 1 yard closer to sensor. Leave the field after walking up to the 1 yard mark. End recording at 10:13:00.

\section{Analysis:}

The motion in this experiment was not side-to-side motion as in the experiments where I walked around in a circle. This was different in that I approached the sensor head-on and then did and about-face and marched away.

The results were curious; it was if the sensor did not even see me coming! The only time it noticed me-and then barely-was when I walked right up to it at 1 yard. The minimum and maximum values should deviate by about 1000 (in Raw Value) when the subject is moving about at 1 yard distance however this was not seen in this particular test. Possible reasons could be the temperature: It was about $55^{\circ}$ Fahrenheit and a little foggy. Or, it could be that this sensor does not respond well when the subject is moving towards-and-away. It works better detecting side-to-side motion.

\begin{tabular}{|r|r|r|r|r|}
\hline & ave 0 & $\min 0$ & $\max 0$ & stddev 0 \\
\hline start & 2059.18 & 1953 & 2182 & 35.98 \\
\hline 7 yds & 2059.03 & 1926 & 2158 & 40.83 \\
\hline 6 yds & 2059.44 & 1905 & 2150 & 39.29 \\
\hline 5 yds & 2059.74 & 1929 & 2162 & 40.34 \\
\hline 4 yds & 2059.23 & 1941 & 2163 & 38.29 \\
\hline 3 yds & 2059.74 & 1945 & 2169 & 40.32 \\
\hline 2 yds & 2060.04 & 1953 & 2150 & 37.44 \\
\hline 1 yd & 2057.44 & 1637 & 2280 & 54.26 \\
\hline
\end{tabular}


Experiment 28 19, July, 2010

Purpose: 90 degrees IR motion sensor distance tests

Sensors: Phidgets 1111 IR motion sensor

Field: Very large open parking lot at Fort Ord base, circular course measured out every 1 yard to 7 yards.

Weather: 55 deg. F. Wind $9 \mathrm{mph}$. Cool, foggy.

Purpose: Test detection capabilities of IR Motion sensors at four compass points. Four tests are conducted, 0, 90, 180 and 270 degrees.

Program: java Sensortest 1052

Implementation:

A set of circular lines was measured and carefully drawn out in heavy chalk on the parking lot surface. With a sensor placement in the center, 7 circles were drawn out. Each circle is 1 yard further out from the center than the previous.

Sensor placed on small box, approximately 5 inches high. Start 17 yards away, (course distance plus 10 yards.) Walk up to a specific yard line on the course and then leave. Do this for each minute of the test. Begin by walking to 7 yard distance circle and then retreating. Each subsequent approach is 1 yard closer. Continue walking 1 yard closer each time, until the 1 yard line.

Synchronize stopwatch with start of test. Begin computer measurements at 10:21:00, at 10:22:00 appear in field and approach the sensor, stopping and turning around at the 7 yard mark. This test is 90 degrees to the sensors, on a compass. Every 60 seconds walk toward the sensor and stop 1 yard closer to sensor than previous. Leave the field after walking up to the 1 yard mark. End recording at 10:29:00.

Analysis: The results are consistent with the previous experiment where the sensors are not very good at detecting motion coming straight towards it. Again if we use a threshold of 1000, the sensor technically did not see me. If we go by the fact that the most change occurred when the subject walked right up to the mark 1 yard away from the sensor and turned around, the change was 357 below the average reading at minimum and 533 above average reading at maximum. If we go by a sensor threshold of 500, the sensor could only detect a human subject at 1 yard. This is far below the advertised 5 meter detection range given in the Phidgets product manual.

\begin{tabular}{|r|r|r|r|r|}
\hline & ave 0 & $\min 0$ & $\max 0$ & stddev 0 \\
\hline start & 2061.1 & 1933 & 2165 & 40.72 \\
\hline 7 yds & 2060.03 & 1949 & 2202 & 46.86 \\
\hline 6 yds & 2059.19 & 1953 & 2182 & 38.73 \\
\hline 5 yds & 2059.1 & 1960 & 2154 & 35.39 \\
\hline 4 yds & 2060.46 & 1966 & 2162 & 29.35 \\
\hline 3 yds & 2059.6 & 1916 & 2198 & 43.34 \\
\hline 2 yds & 2061.2 & 1897 & 2185 & 40.67 \\
\hline 1 yd & 2058.49 & 1701 & 2591 & 78.21 \\
\hline
\end{tabular}


Experiment 29 2, July, 2010

Purpose: 180 degrees IR motion sensor distance tests

Sensors: Phidgets 1111 IR motion sensor

Field: Very large open parking lot at Fort Ord base, circular course measured out every 1 yard to 7 yards.

Weather: 55 deg. F. Wind $9 \mathrm{mph}$. Cool, foggy.

Purpose: Test detection capabilities of IR Motion sensors at four compass points. Four tests are conducted, 0, 90, 180 and 270 degrees.

Program: java Sensortest 1052

Implementation:

A set of circular lines was measured and carefully drawn out in heavy chalk on the parking lot surface. With a sensor placement in the center, 7 circles were drawn out. Each circle is 1 yard further out from the center than the previous.

Sensor placed on small box, approximately 5 inches high. Start 17 yards away, (course distance plus 10 yards.) Walk up to a specific yard line on the course and then leave. Do this for each minute of the test. Begin by walking to 7 yard distance circle and then retreating. Each subsequent approach is 1 yard closer. Continue walking 1 yard closer each time, until the 1 yard line.

Synchronize stopwatch with start of test. Begin computer measurements at 10:34:00, at 10:35:00 appear in field and approach the sensor, stopping and turning around at the 7 yard mark. This test is 180 degrees to the sensors, on a compass. Every 60 seconds walk toward the sensor and stop 1 yard closer to sensor than previous. Leave the field after walking up to the 1 yard mark. End recording at 10:41:00.

Analysis: Like experiments $27 \& 28$, the sensitivity for the IR motion detector only works out to 1 yard when a human subject walks towards-then-away from the sensor. The test at 1 yards shows a change in raw value over a threshold of 500. If we are using a threshold value of 1000 then the sensor would technically not detect anything in this case.

\begin{tabular}{|r|r|r|r|r|}
\hline & ave 0 & $\min 0$ & $\max 0$ & stddev 0 \\
\hline start & 2059.49 & 1949 & 2186 & 36.98 \\
\hline 7 yds & 2060.01 & 1921 & 2165 & 40.44 \\
\hline 6 yds & 2060.23 & 1949 & 2189 & 39.37 \\
\hline 5 yds & 2059.23 & 1945 & 2190 & 41.72 \\
\hline 4 yds & 2060.27 & 1970 & 2150 & 33.12 \\
\hline 3 yds & 2061.56 & 1965 & 2198 & 35.62 \\
\hline 2 yds & 2059.13 & 1961 & 2174 & 40.64 \\
\hline 1 yd & 2058.17 & 1417 & 2850 & 95.49 \\
\hline
\end{tabular}


Experiment 30 2, July, 2010

Purpose: 270 degrees IR motion sensor distance tests

Sensors: Phidgets 1111 IR motion sensor

Field: Very large open parking lot at Fort Ord base, circular course measured out every 1 yard to 7 yards.

Weather: 55 deg. F. Wind $9 \mathrm{mph}$. Cool, foggy.

Purpose: Test detection capabilities of IR Motion sensors at four compass points. Four tests are conducted, 0, 90, 180 and 270 degrees.

Program: java Sensortest 1052

Implementation:

A set of circular lines was measured and carefully drawn out in heavy chalk on the parking lot surface. With a sensor placement in the center, 7 circles were drawn out. Each circle is 1 yard further out from the center than the previous.

Sensor placed on small box, approximately 5 inches high. Start 17 yards away, (course distance plus 10 yards.) Walk up to a specific yard line on the course and then leave. Do this for each minute of the test. Begin by walking to 7 yard distance circle and then retreating. Each subsequent approach is 1 yard closer. Continue walking 1 yard closer each time, until the 1 yard line.

Synchronize stopwatch with start of test. Begin computer measurements at 11:06:00, at 11:07:00 appear in field and approach the sensor, stopping and turning around at the 7 yard mark. This test is 270 degrees to the sensors, on a compass. Every 60 seconds walk toward the sensor and stop 1 yard closer to sensor than previous. Leave the field after walking up to the 1 yard mark. End recording at 11:14:00.

Analysis: The sensors performed even worse in this experiment than the previous three! If we go by the raw value threshold of 500 , the sensor did not detect motion at all. The best readings were still at the 1 yard mark, yet the change was only about 300 . This seems to solidify the conclusion that while the sensors can detect motion in 360 degrees, this particular type of motion is difficult for the sensor to detect. This could be considered an exploit if someone were to walk up close to a monitoring node (a station in a wireless sensor network) and drop an explosive device and retreat swiftly.

\begin{tabular}{|r|r|r|r|r|}
\hline & ave 0 & $\min 0$ & $\max 0$ & stddev 0 \\
\hline start & 2060.84 & 1877 & 2182 & 50.11 \\
\hline 7 yds & 2063.44 & 1949 & 2194 & 45.88 \\
\hline 6 yds & 2060.95 & 1901 & 2194 & 46.13 \\
\hline 5 yds & 2061.86 & 1901 & 2193 & 45.05 \\
\hline 4 yds & 2060.88 & 1969 & 2177 & 38.06 \\
\hline 3 yds & 2060.60 & 1905 & 2194 & 48.27 \\
\hline 2 yds & 2060.92 & 1926 & 2210 & 48.91 \\
\hline 1 yd & 2061.91 & 1861 & 2241 & 56.13 \\
\hline
\end{tabular}




\section{Appendix B}

\section{Quad experiments analysis}

\section{B.1 Infrared Motion Sensor Analysis}

Experiment 1 the subject simply walked from one end of the quad to the other and back again. As shown in the previous quad-map, the subject starts near sensor 4. Their path is 4, 3,2,1 and $1,2,3,4$. The following graph demonstrates that we can track the subject as they walk through the quad by using the IR motion sensor. A program was written to accept raw-data-files from the sensors, representing sensor locations on the map. The program can analyze a specific sensor (in this case the data column corresponding to the IR motion sensor,) find the arithmetic mean for that sensor at each location, and then choose rows in the raw-data that are above/below a specific sensor threshold. From earlier experiments, we find the IR Motion sensor reads approx. 2000 when nothing is in view. For this graph, a threshold of 500 was chosen.

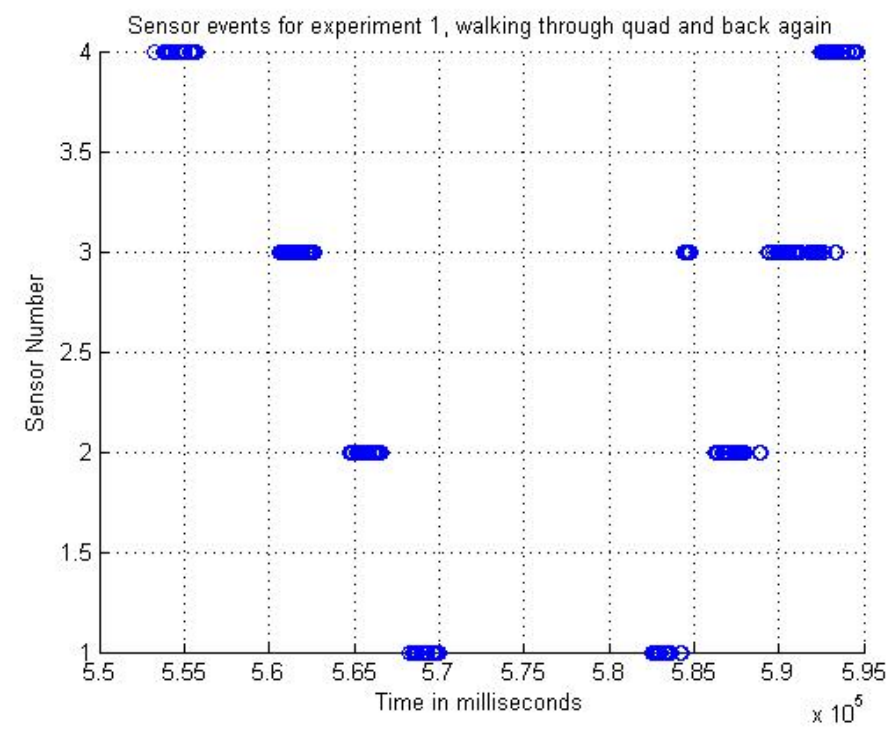

Each square represents approximately 5 seconds of time along the $\mathrm{X}$ axis. Looking at the above graph we can see the subject walked past sensors $4,3,2,1$, and took about 2 seconds to do so. The odd blip from sensor 3 near time 585000 milliseconds could be one of several things. In previous experiments we found that our wires were blowing in the breeze, so we had to tape them down. This blip could either be a moving wire, or wind blowing the actual sensor. Since the quad area is planted with Chinese Pistachio trees, this blip could be moving leaves or a bird flying by. It is also possible that sensor 3 is just a bit more "sensitive" than others and may need to be tuned down. Please see 
the section on False Positives of Sensors at the end of this chapter. 
Experiment 2: The subject walks from sensor 4 to the center of the sensor field and makes a left turn, then continues past sensor 9 . Their path is $4,3,2,7,6,5$. The graph below is very similar to the graph from experiment 1 and shows a subject moving past the respective sensors in order. Again, the sensor graphed was the IR Motion sensor, and the threshold set at 500 .

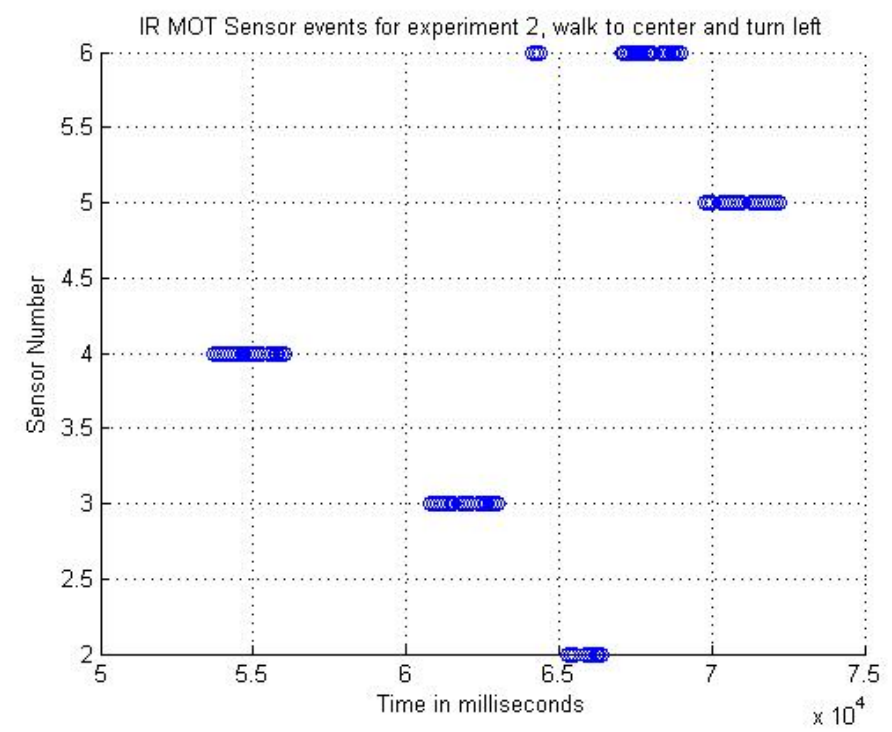

Sensor 6 shows a small blip, but it may still be the same subject. The sensors in the quad were placed approximately 12 feet apart, and the subject was near \#6 and \#2 at the same time. The approximate range of the IR motion sensor is 4 yards, using a threshold of 500 . The squares on the map of the Glasgow Hall quad are 6 feet wide, thus the distance between sensors 2 \& 6 is 12 feet, the effective edge of the IR motion sensor's range. Notice that sensor 7 does not appear on this graph. Sensor 7 had no raw data values above the threshold in this experiment. 
Experiment 3 shows a subject following the same path as in Exp. 2, however suspicious motion is introduced. The subject carries a bag and walks from sensor 4 to the center of the sensor field and makes a left turn, then continues to a position near sensor 6 where there is a force sensor run by 6 , then places the bag on the force sensor. They then walk past sensor 9 .

The graph below shows a clear delay of the subject at location 6 . This is an example of tracking someone and detecting suspicious behavior.

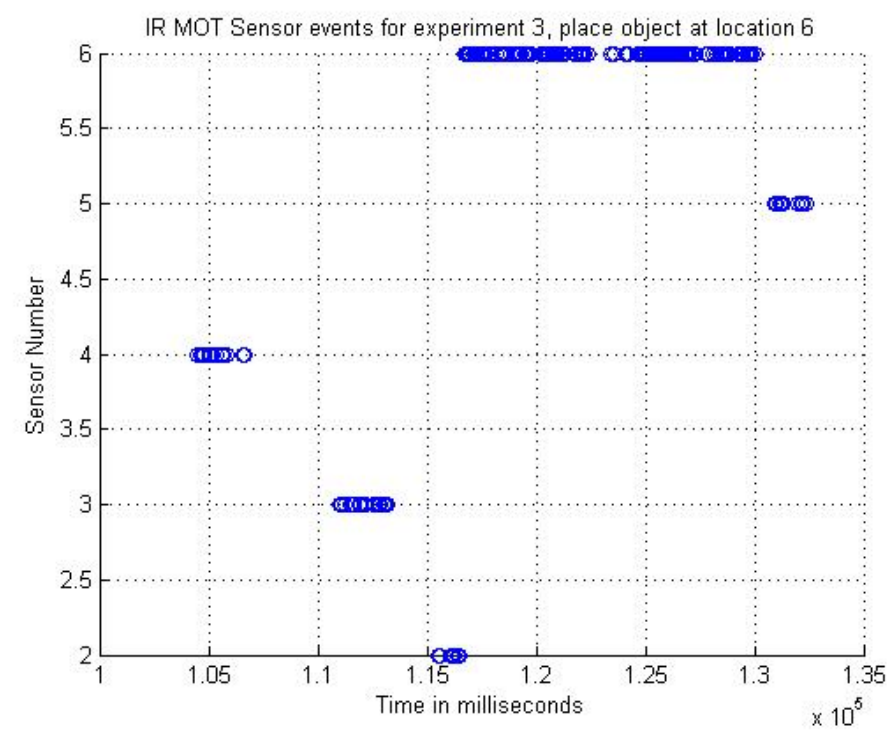

The subject's path was: $4,3,2,6,5$. This graph shows the subject spent about 14 seconds placing the bag at location 6 . Clusters of data points reported by a sensor are indicative of suspicious motion. Tracking is not absolutely necessary for detecting suspicious motion. The subject could have dropped out of the sky, (not a joke, imagine a paratrooper landing in a sensor-monitored zone,) and if they linger long enough to dig a hole and place an IED, a nearby sensor will detect loitering in a well built system. 
Experiment 4: This graph s a bit more chaotic. It represents similar suspicious behavior as seen in the previous experiment. This time the subject retrieves the bag previously dropped. Otherwise the path is identical to that of Experiment 4.

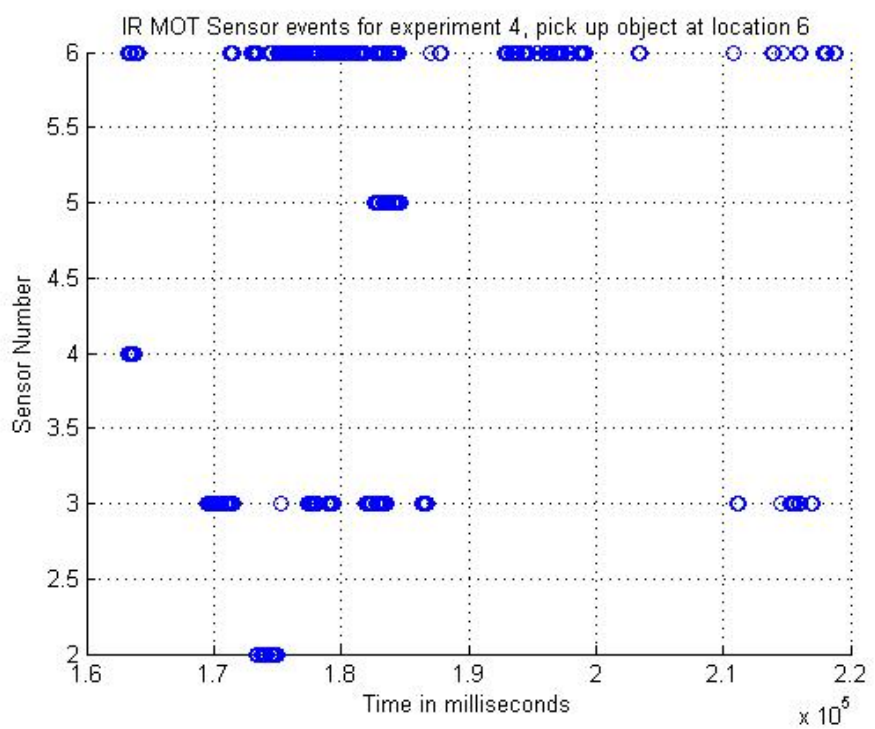

Sensor 6 picks up a lot more loitering in this data. While the graph may look chaotic, this is actually helpful; a chaotic graph with clustering around a particular sensor is also indicative of suspicious behavior.

Possible errors: We know the subject's path began at sensor 4, yet sensor 6 also picked up motion at this time. This may be an artifact of trees blowing in the wind, and sensor 6 picking up the motion. Possible solution: Extra activity at a certain sensor may be averaged out, and loitering can still be detected if the sensor shows readings above and beyond the previously-accounted-for wind motion. 
Experiment 5: This graph shows a subject loitering. The subject walks to the center of the sensor field, then to the area of sensors 5-7, and loiters within a circle of a ten foot radius for 30 seconds, then walks off past sensor 9 . The graph of IR motion is even more chaotic than the previous experiment.

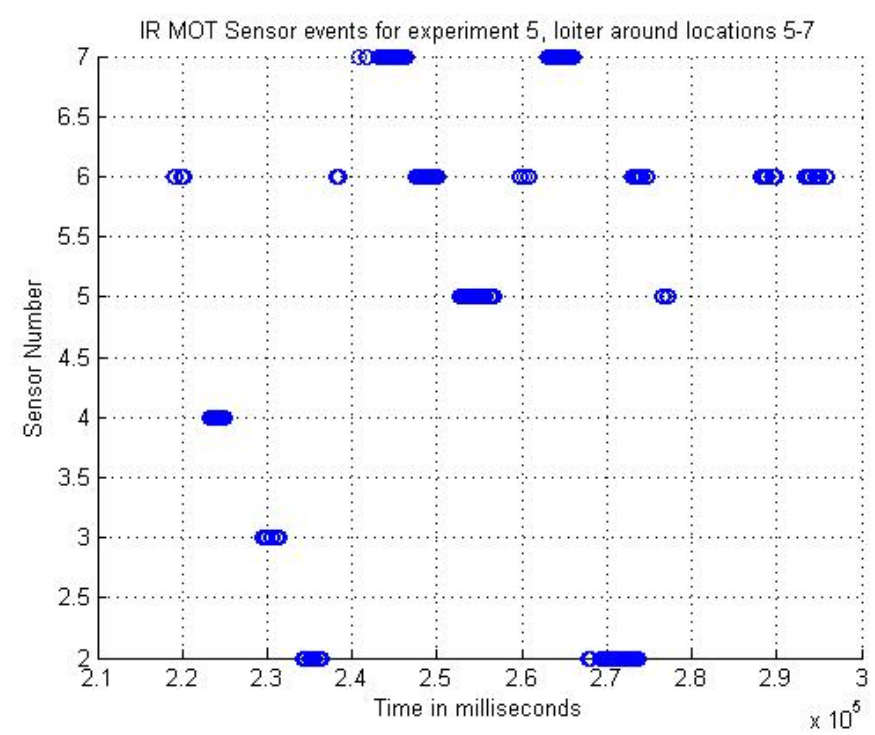

One may interpret the subject's path as $4,3,2,6,7 \ldots$ with a wandering back and forth between 5 and 7 as the experiment script states. This bouncing motion on the graph can indicate someone is wandering around and not following a typical path. Traffic patterns should be analyzed wherever sensor arrays are deployed, so observers can tell between normal and suspicious behavior. This meandering in a specific sensor area-as seen by the repeat readings around sensors $5,6, \&$-indicates suspicious motion. 
Experiment 6 This experiment is similar to the previous one, however the subject loiters in a larger radius around sensors 5 and 7 .

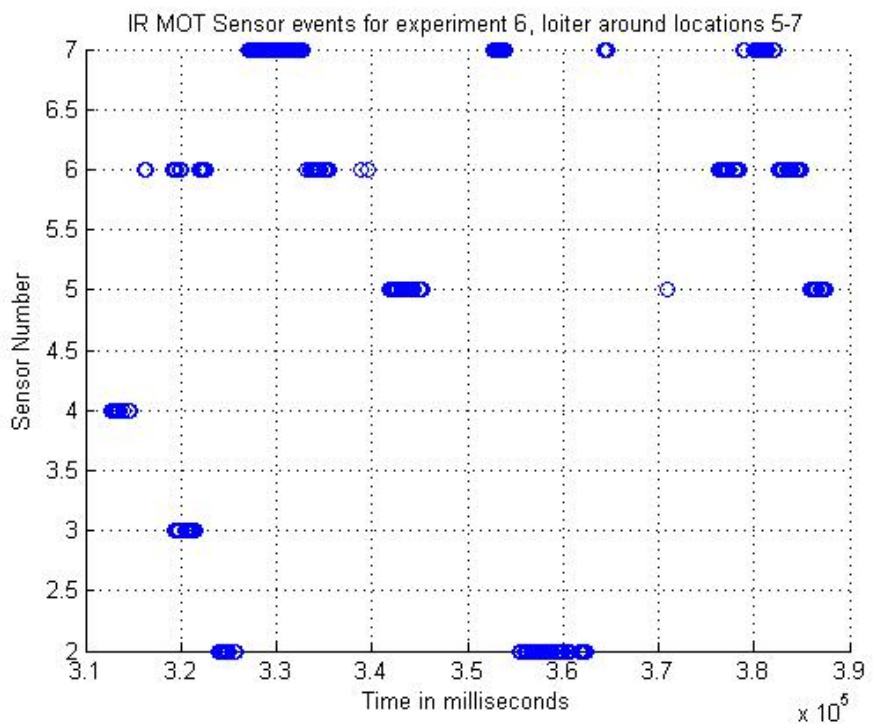

We see an initial path from 4, 3, 2, then a lot of chaotic behavior between sensors 5 and 7 where the subject was loitering. This indicates the IR Motion sensors are good at detecting when someone is moving about erratically in an area; not following a typical path for the area is an example of suspicious behavior. 
Experiment 7 The subject walks to location 5 and inserts a shovel five times into the ground. This was done to simulate digging, as someone planting an IED might do.

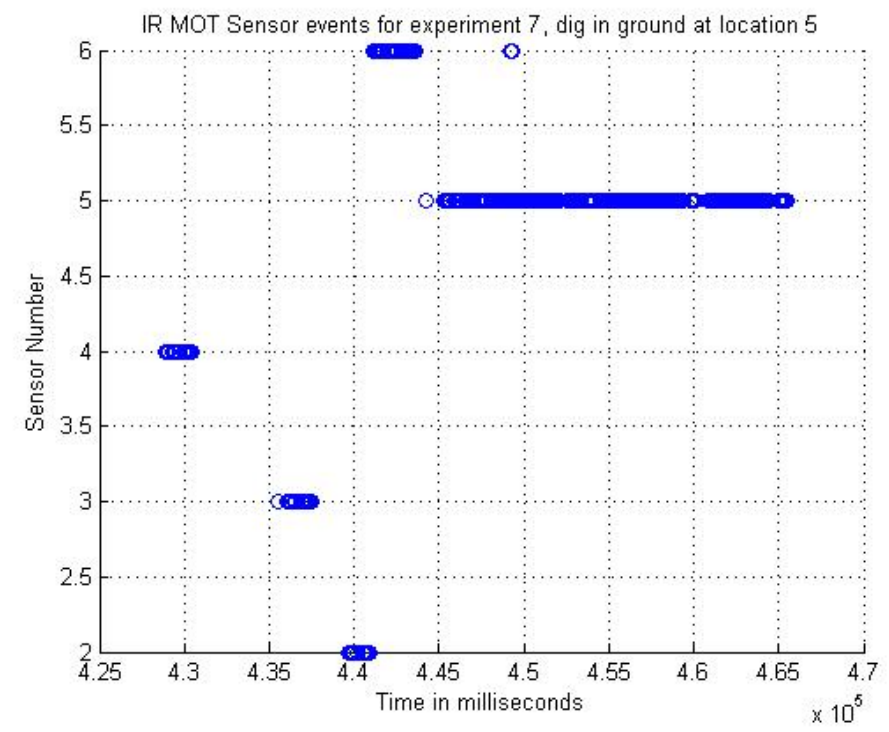

This graph illustrates a well defined clustering of points around sensor 5. The individual spent approximately 22 seconds in the area and then moved on. If we follow the motion from sensor 4 to 3 to 2 , we see the subject is walking along at a regular speed in a non-suspicious direction. We expect this path. However, they deviate from non-suspicious behavior by loitering at 5 . Changes in goal by a subject (such as walking then stopping) indicate suspicious behavior. 
Experiment 8: One subject walks to the center of the sensor field, then loiters within a 10 foot radius of sensors 5-7. Another subject walks across the sensor field from top to bottom.

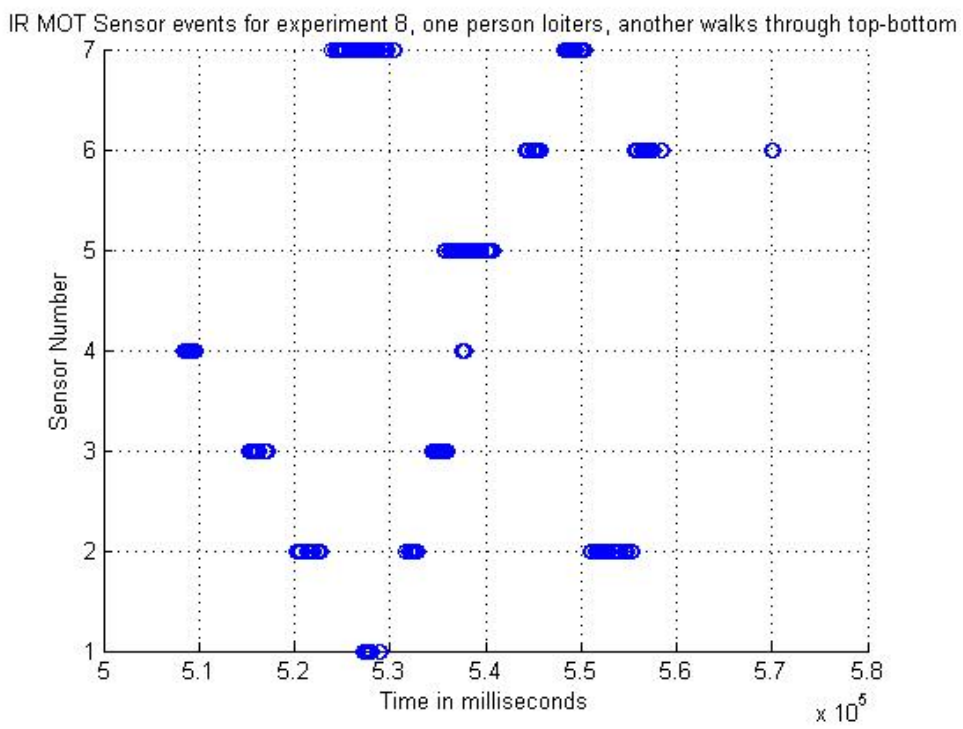

The path from top-to-bottom is $1,2,3,4$. We can see the non-suspicious subject walking through at around time 5.3 in the graph. The graph continues to show motion around 7 and 5 during this time. Graphs with multiple subjects may be difficult to interpret. We do pick up the motion from both subjects in the graph, however we would need to know which patterns of data are normal (such as the path $1,2,3,4)$ and remove those from the graph to see the remaining behavior is suspicious. 
Experiment 9: Similar to number 8, however the non-suspicious subject walks from right to left while a suspicious subject is loitering.

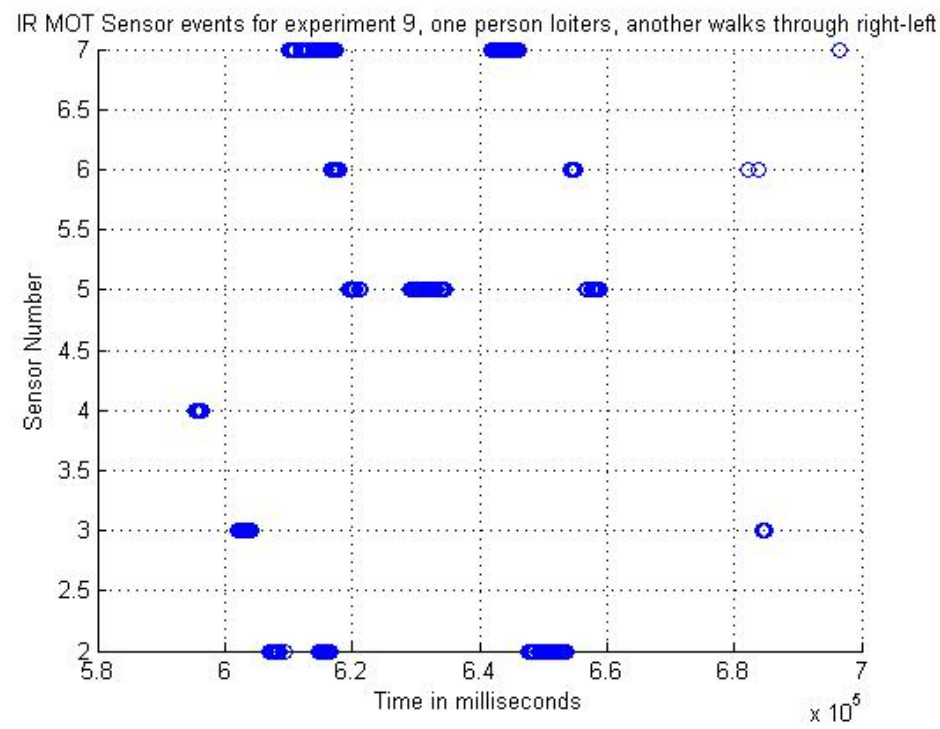

Judging by the chaotic action of the plots around sensors 5 and 7, we can see our suspicious subject was loitering in the area. 
Experiment 10: One subject walks from the bottom of the sensor field to the top, dragging a shovel. (This was mainly for the benefit of the microphones attached to the laptops.)

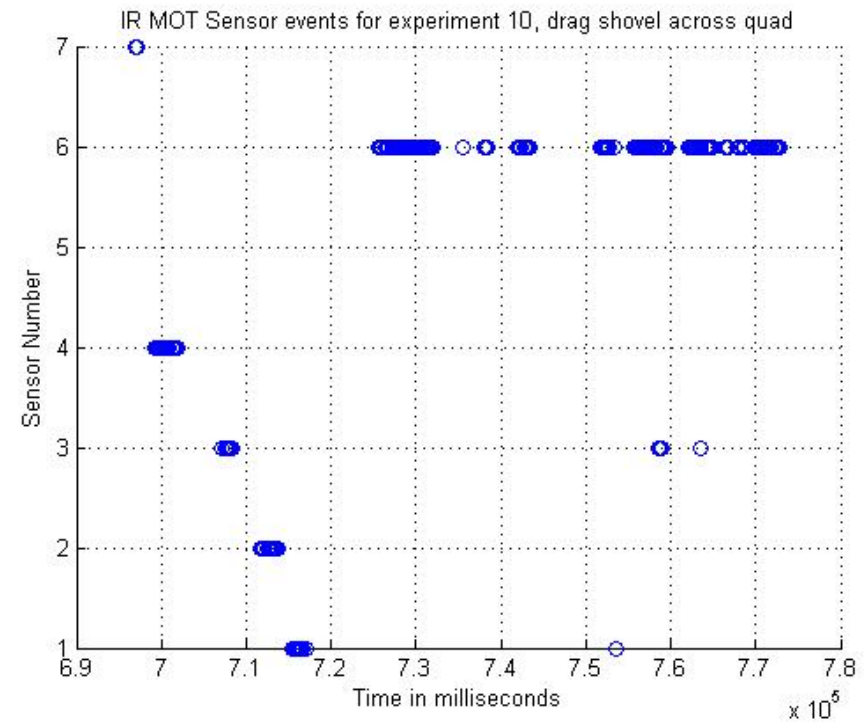

While this experiment shows a clear path from 4, 3, 2, 1, it also shows a long streak of data around location 6 . Nobody was around location 6 during this time, so it may be a motion-sensor picking up the wind blowing the leaves of the red-bud trees planted in the quad. In setting up a system, one must analyze the field when no suspicious activity is occurring in order to determine if any sensors need to be de-tuned. Placing motion sensors near trees that are going to get blown by the wind (NPS is 1 block away from the beach) is not recommended, as the motion sensors will simply report every time the wind blows. Please see the following appendix section on False Positives of Sensors. 


\section{B.2 Infrared Distance Sensors}

Experiment 1: This is a non-suspicious experiment. Simply walk across the quad and back.

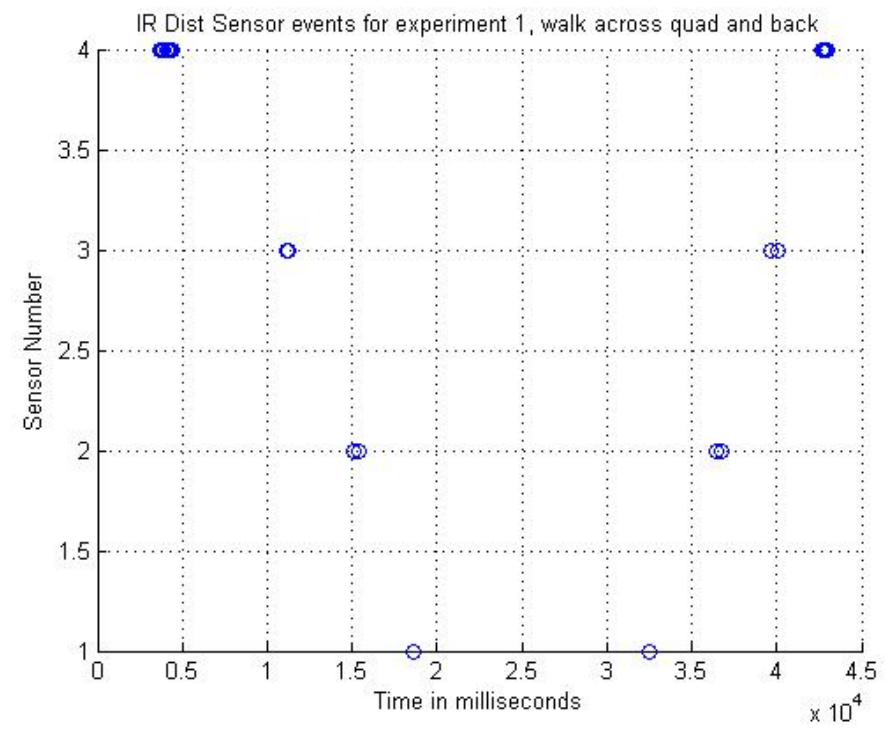

The IR distance sensors act like an electric-eye or a door-beam in a retail store. They are a narrow beam and they report in a binary fashion when an object crosses the beam. It is clear to see a subject walking from 4, 3, 2, 1 and then 1,2, 3, 4 along a straight-line path. 
Experiment 2: Walk to center of quad and turn left. Non-suspicious activity.

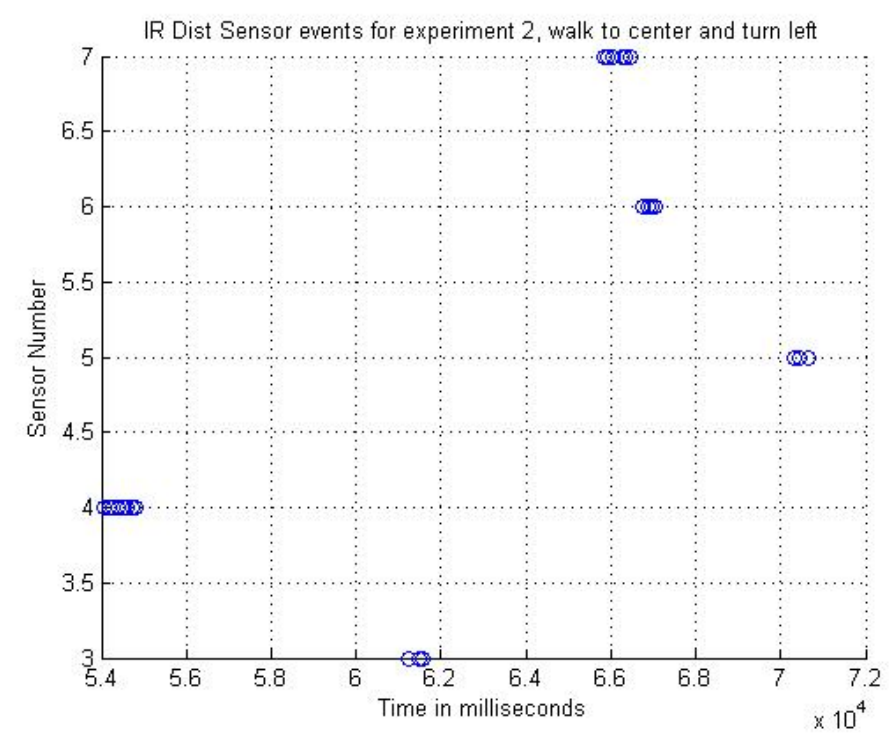

The path here is along $4,3,2,7,6,5$. There are no clusters of data or bouncing-patterns (going back and forth between 2 or more sensors) to indicate that a subject meandered in an area. 
Experiment 3: Drop a bag at location 6.

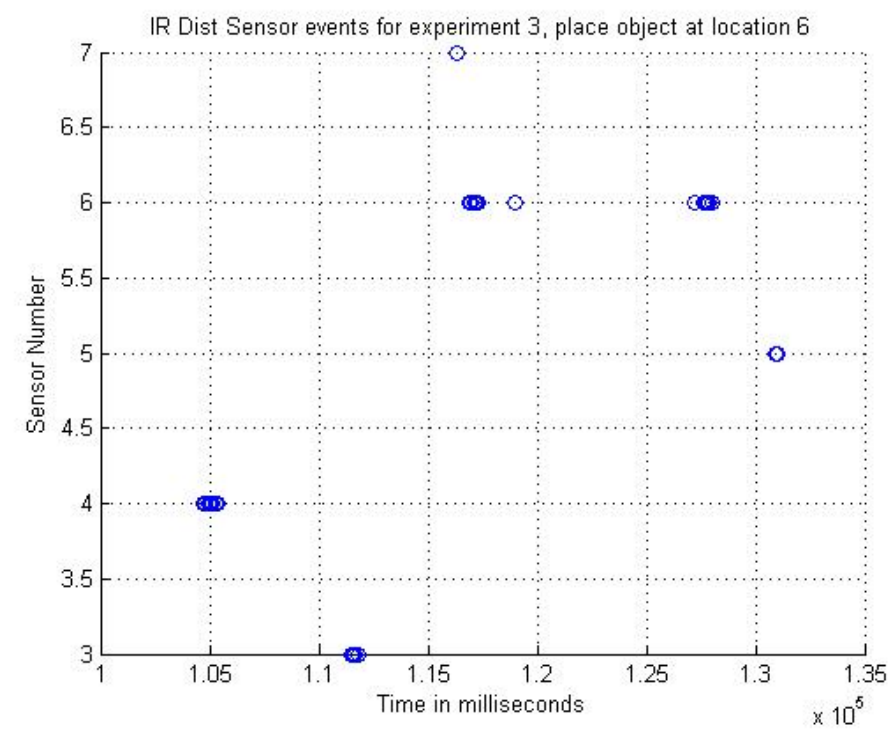

This shows how the subject crossed the beam at location 6 more than once. It can be used to show loitering in the area. We can see the subject spent a little over 10 seconds in the area if we use both clusters of data points at location 6 as book-ends for their passage through the area. 
Experiment 4: Pick up a bag at location 6 .

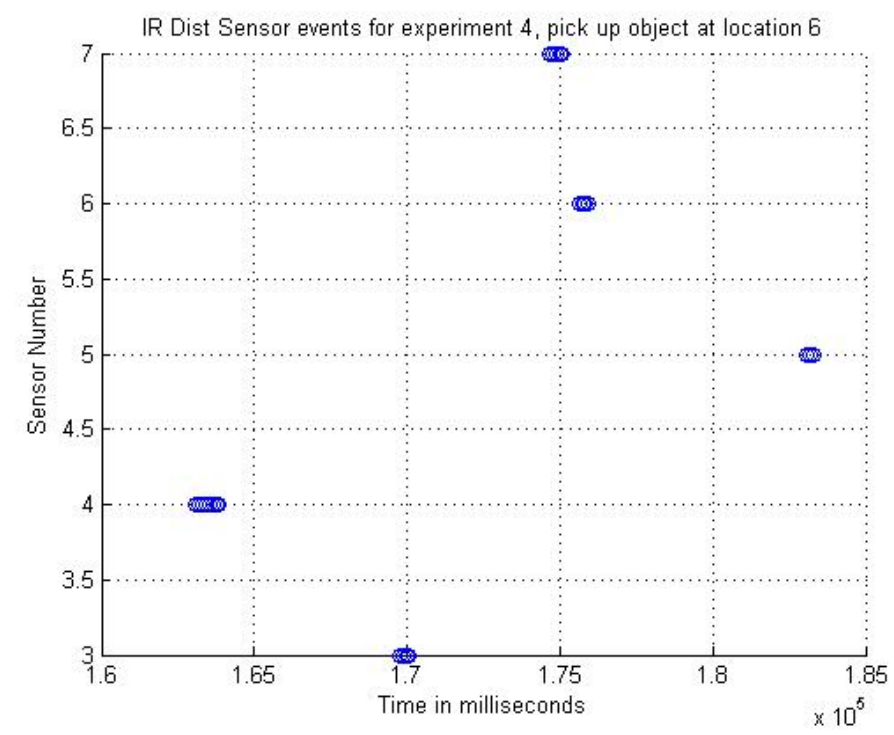

This one is more difficult to tell suspicious behavior. We may want to link points together with a length of time deemed normal for passage through the area. When points are spaced too far apart in time, that could indicate loitering. The IR distance sensor has a long, narrow field of vision. Reports by this sensor should be thought of as someone crossing a beam, similar to the ones found at doors in retail shops. 
Experiment 5: Walk to center of quad and loiter around locations 5-7.

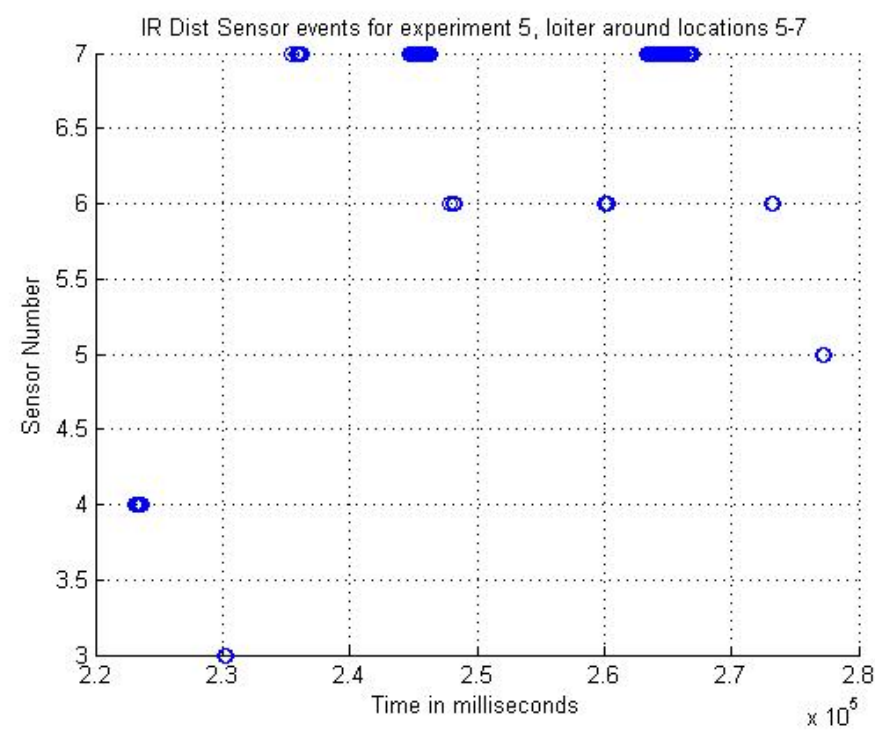

This shows how a suspicious subject meandered around points 5 and 7 without making a clear path through the field. 
Experiment 6: Same as experiment 5, with a larger area of loitering around 5-7.

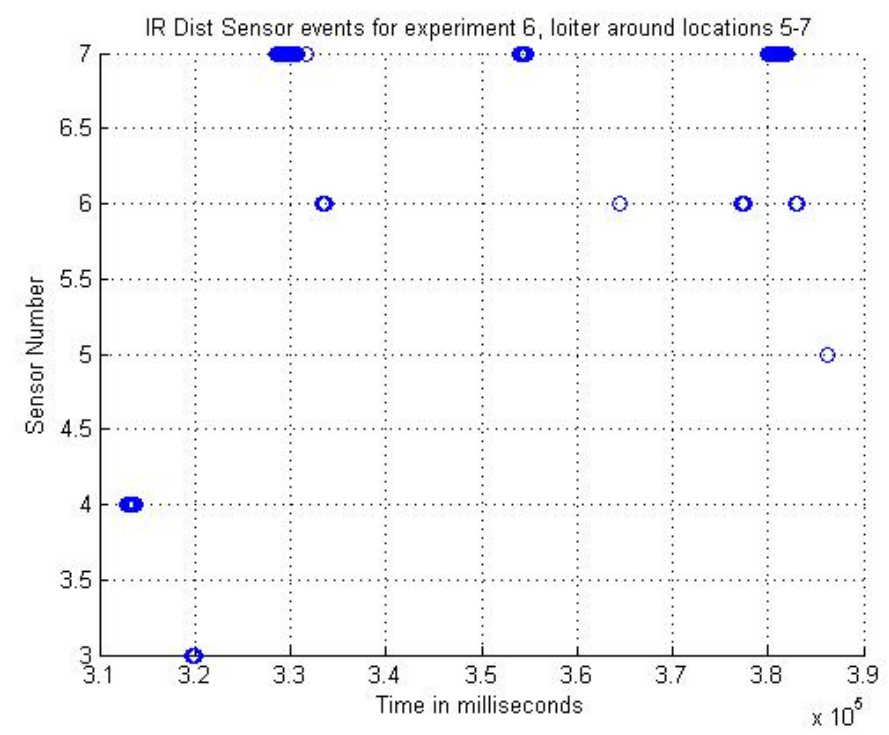

Suspicious activity can be seen around 5-7. When bouncing is seen on the graph, it indicates a subject traveled back and forth between sensors. This is an example of meandering behavior. Repeated over-threshold hits on a sensor indicate that someone did not simply walk past the sensor once as if they were on their way somewhere. It shows that they passed by the same location multiple times and is not normal behavior for the area. 
Experiment 7: Walk to center of quad and turn left. Stop at location 5 and dig in the ground, (with 5 shovel hits.)

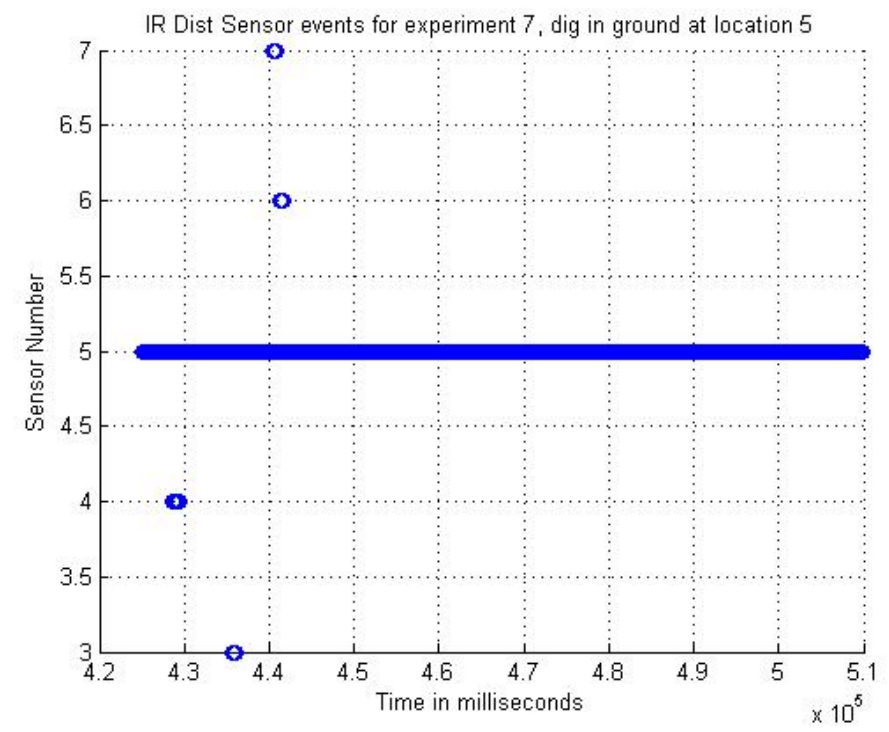

This graph shows that the subject left an object in front of the sensor. When I was doing this experiment, I forgot that I was supposed to take the shovel with me after digging! Even though I made a mistake, it illustrates very well that the IR distance sensors can detect object placement. Any time we see clustering of data points where there was no previous activity, we have detected suspicious behavior. An object being left at the scene is certainly suspicious. 
Experiment 8: One subject loiters around locations 5-7. Another subject walks through the quad from top to bottom.

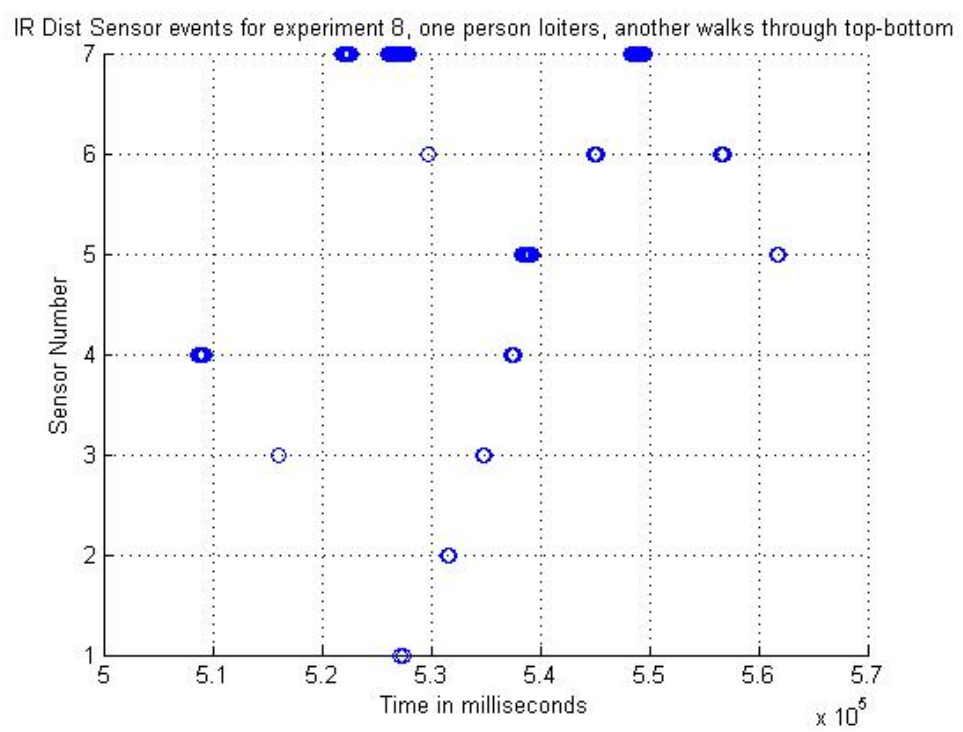

This graph looks like the graph for the IR Motion sensors experiment 8, just with tighter point clustering. 
Experiment 9: Similar action to experiment 8, with one subject loitering around locations 5-7. Another subject walks through the quad from right to left.

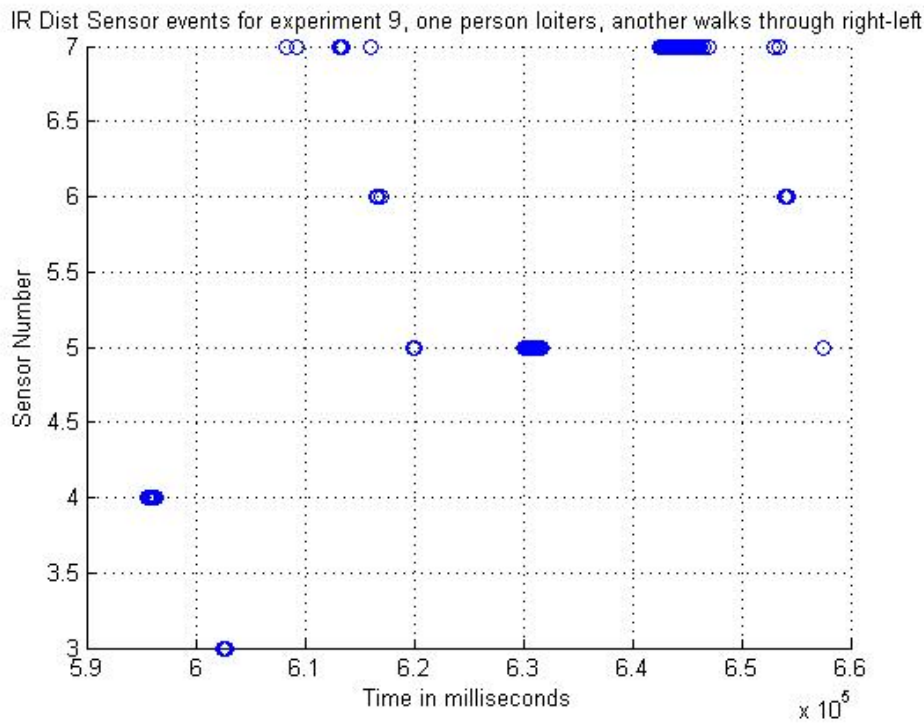

Suspicious loitering and meandering occurs around locations 5-7. 
Experiment 10: Walk through quad dragging a shovel.

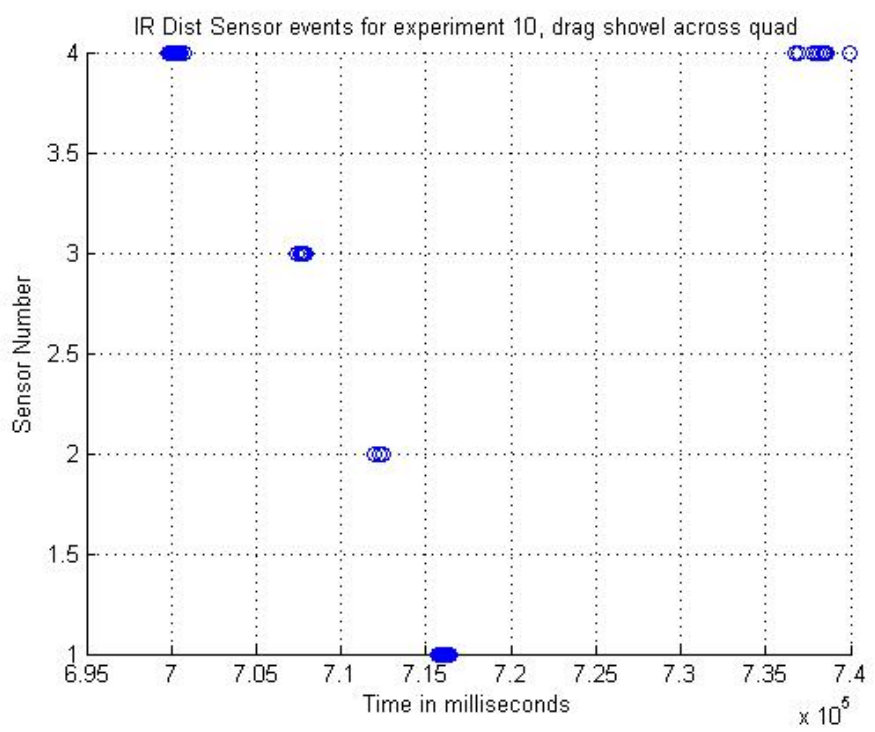

This shows a nice path from $4,3,2,1$. There is extra data at the end, on location 4 . The video tape verifies that my colleague Riqui entered the sensor area at location 4 to put his experiment script down on a curb, after I had walked through the area with the shovel. 


\section{B.3 Sonar Distance Sensors}

\section{What Works and What Doesn't}

Previous analysis of sensors relied on finding the arithmetic mean for the sensor at that location. The arithmetic mean is basically the sensor's reading at rest with no activity detected. Analysis was done by selecting a threshold range and throwing out all tuples that were under that specific number. In other words, if a sensor shows 2000 at rest and we set up a bar of 500 points, and change that doesn't make it over the bar is ignored. We only report the tuples that made it over the bar, (exceeded a change of 500 points from the Arithmetic Mean.) This works for sensors like the Infrared Motion and distance sensors, but it does not work for the sonar sensors. The various infrared sensors go back to a resting state when no objects are in view, and that resting state varies slightly between experiments. Sometimes it is 1999, sometimes it is 2018, etc. The sonar sensors do not behave that way, unless they are pointing at the sky and are totally unobstructed.

\section{Sonar sensors each had a different at-rest value}

Analysis of the sonar sensors showed that each of them had their own different arithmetic mean reading, and none of them were showing infinity (lack of obstruction) in their views. Since they can all see out to 5 yards, this means that some object was closer than that for each sensor in the experiment. Since they all reported something in the way we can't say, "report only those tuples with a change greater than 500 raw data points." There will never be a change of greater than 500 if the arithmetic mean for a sensor is 350 . Therefore it is erroneous to rely on the fact that they all read 2000 at rest. They do when they are pointed at the sky, but not in experimentation. The following tables describe the Sonar Sensor behavior during the experiments at each location:

\section{Location 1 Sonar:}

\begin{tabular}{|r|r|r|r|}
\hline Exp \# & $\min$ & $\max$ & mean \\
\hline 1 & 172 & 2045 & 1139.41 \\
\hline 2 & 166 & 2042 & 1128.73 \\
\hline 3 & 163 & 2048 & 1041.56 \\
\hline 4 & 170 & 2047 & 1251.99 \\
\hline 5 & 160 & 2048 & 1157.27 \\
\hline 6 & 163 & 2045 & 1124.87 \\
\hline 7 & 168 & 2050 & 1205.24 \\
\hline 8 & 168 & 2046 & 1064.63 \\
\hline 9 & 166 & 2048 & 1127.82 \\
\hline 10 & 173 & 2043 & 1225.88 \\
\hline average & & & 1146.74 \\
\hline
\end{tabular}




\section{Location 2 Sonar:}

\begin{tabular}{|r|r|r|r|}
\hline Exp \# & $\min$ & $\max$ & mean \\
\hline 1 & 224 & 2022 & 513.89 \\
\hline 2 & 200 & 2012 & 751.58 \\
\hline 3 & 168 & 2022 & 692.25 \\
\hline 4 & 176 & 2022 & 678.35 \\
\hline 5 & 76 & 2022 & 582.72 \\
\hline 6 & 100 & 2025 & 624.94 \\
\hline 7 & 156 & 2020 & 671.44 \\
\hline 8 & 104 & 2024 & 486.34 \\
\hline 9 & 112 & 2022 & 602.58 \\
\hline 10 & 124 & 2022 & 778.56 \\
\hline average & & & 638.27 \\
\hline
\end{tabular}

Location 3 Sonar:

\begin{tabular}{|r|r|r|c|}
\hline Exp \# & $\min$ & $\max$ & mean \\
\hline 1776 & 1974 & 217.09 & \\
\hline 2 & 92 & 1637 & 209.37 \\
\hline 3 & 76 & 1661 & 225.32 \\
\hline 4 & 104 & 1977 & 220.82 \\
\hline 5 & 76 & 1951 & 197.41 \\
\hline 6 & 80 & 1856 & 210.34 \\
\hline 7 & 80 & 2035 & 237.51 \\
\hline 8 & 75 & 1949 & 219.00 \\
\hline 9 & 84 & 1801 & 207.34 \\
\hline 10 & 87 & 1985 & 213.29 \\
\hline average & & & 215.75 \\
\hline
\end{tabular}

Location 4 Sonar:

\begin{tabular}{|r|r|r|r|}
\hline Exp \# & $\min$ & $\max$ & mean \\
\hline 1 & 87 & 1418 & 527.31 \\
\hline 2 & 100 & 1816 & 536.25 \\
\hline 3 & 75 & 1770 & 528.22 \\
\hline 4 & 92 & 1367 & 528.68 \\
\hline 5 & 86 & 1455 & 523.87 \\
\hline 6 & 87 & 1440 & 519.66 \\
\hline 7 & 78 & 1810 & 526.98 \\
\hline 8 & 87 & 1265 & 525.48 \\
\hline 9 & 79 & 1480 & 533.43 \\
\hline 10 & 84 & 1487 & 520.53 \\
\hline average & & & 527.04 \\
\hline
\end{tabular}




\section{Location 5 Sonar:}

\begin{tabular}{|r|r|r|r|}
\hline Exp \# & $\min$ & $\max$ & mean \\
\hline 1 & 81 & 359 & 314.24 \\
\hline 2 & 80 & 357 & 328.24 \\
\hline 3 & 81 & 358 & 311.48 \\
\hline 4 & 83 & 359 & 330.23 \\
\hline 5 & 83 & 359 & 306.80 \\
\hline 6 & 81 & 364 & 312.79 \\
\hline 7 & 64 & 358 & 279.32 \\
\hline 8 & 58 & 356 & 321.96 \\
\hline 9 & 82 & 361 & 323.23 \\
\hline 10 & 82 & 369 & 321.10 \\
\hline average & & & 314.94 \\
\hline
\end{tabular}

Location 6 Sonar:

\begin{tabular}{|r|r|r|r|}
\hline Exp \# & $\min$ & $\max$ & mean \\
\hline 1 & 132 & 2029 & 1028.14 \\
\hline 2 & 140 & 2022 & 1026.18 \\
\hline 3 & 67 & 2026 & 953.04 \\
\hline 4 & 67 & 2023 & 1056.28 \\
\hline 5 & 124 & 2037 & 1092.61 \\
\hline 6 & 121 & 2031 & 1097.55 \\
\hline 7 & 128 & 2030 & 1009.86 \\
\hline 8 & 84 & 2040 & 1048.75 \\
\hline 9 & 124 & 2032 & 1023.75 \\
\hline 10 & 128 & 2033 & 1139.53 \\
\hline average & & & 1047.57 \\
\hline
\end{tabular}

Location 7 Sonar:

\begin{tabular}{|r|r|r|r|}
\hline Exp \# & $\min$ & $\max$ & mean \\
\hline 1 & 216 & 1068 & 259.23 \\
\hline 2 & 80 & 896 & 247.24 \\
\hline 3 & 80 & 1658 & 254.34 \\
\hline 4 & 216 & 1460 & 264.73 \\
\hline 5 & 52 & 1580 & 257.59 \\
\hline 6 & 51 & 1421 & 258.51 \\
\hline 7 & 80 & 1272 & 253.19 \\
\hline 8 & 56 & 1640 & 239.41 \\
\hline 9 & 60 & 1297 & 237.14 \\
\hline 10 & 72 & 1080 & 238.21 \\
\hline average & & & 250.96 \\
\hline
\end{tabular}




\section{Notes on Sonar:}

Houston, we have a problem!

Locations3, 5 and 7 each report an object in close proximity. There could be a few reasons for this, such as ground reflection, or more likely that an object was within range like a tree or a picnic bench. Location 3 had an average reading of 215.75 , location 5 had an average of 314.94 and location 7 showed an average of 250.96. That basically means something was 1 or 2 yards away from each sonar sensor.

As we can see from location 7 , the average reading is 250.96 which indicates something is approximately 1 yard in front of the sensor. Sensors were placed on plastic blocks 6 inches up, however it is possible this particular was getting ground reflection of some kind.

Even when we choose a static threshold of 250 , that is we only graph points where the threshold is 250 regardless or arithmetic mean of the sensor, we find regular patterns of noise. We see many false positives where no subject was in the field. 250 was chosen as the value a sonar sensor should show when an object is placed 1 yard away.

Graph of Experiment 1 with Sonar Sensor threshold static at 250:

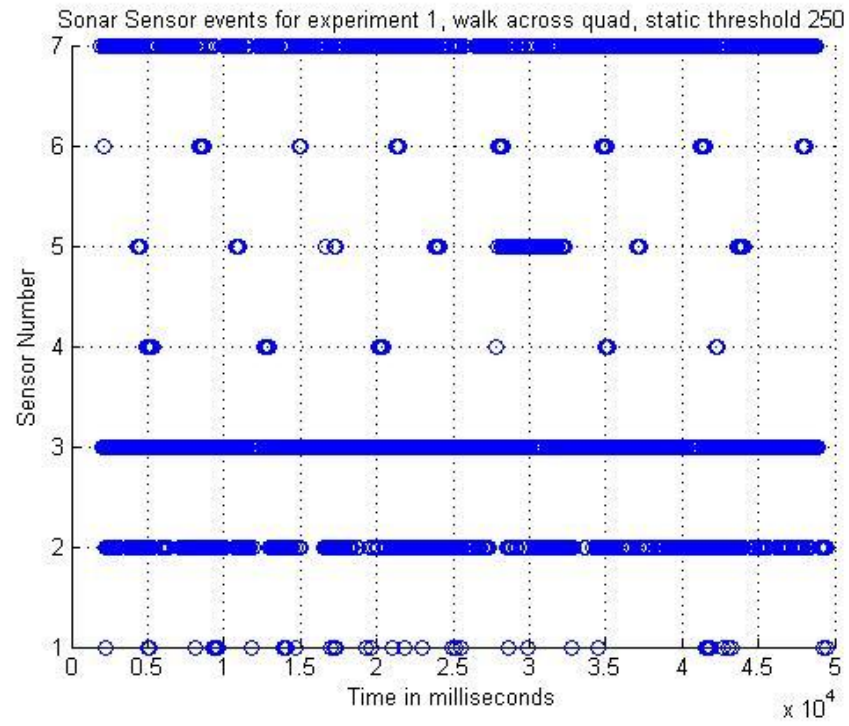


Graph of Experiment 2 with Sonar Sensor threshold static at 250:

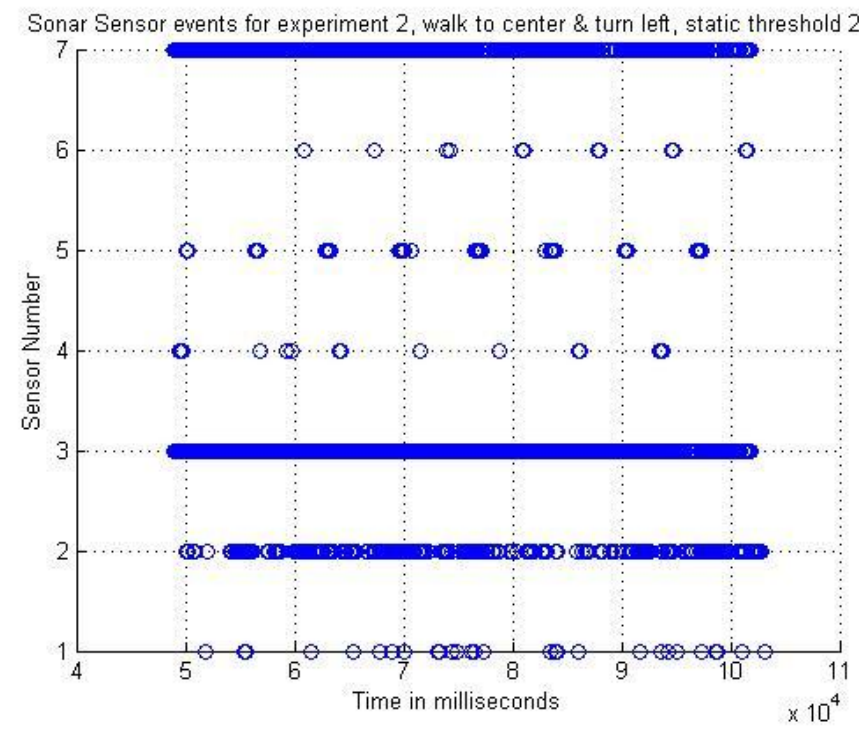

Lowering the threshold to 150 still shows problematic false positives and regular noise. 150 should be closer than 1 yard from the sensor. Graph of Experiment 1 with Sonar Sensor threshold static at 150:

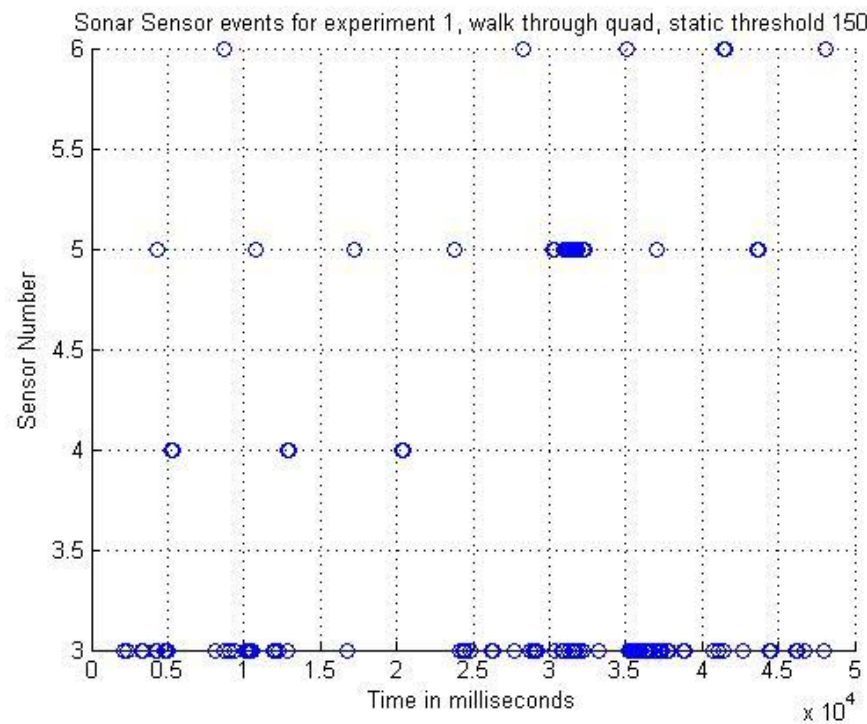


Graph of Experiment 2 with Sonar Sensor threshold static at 150:

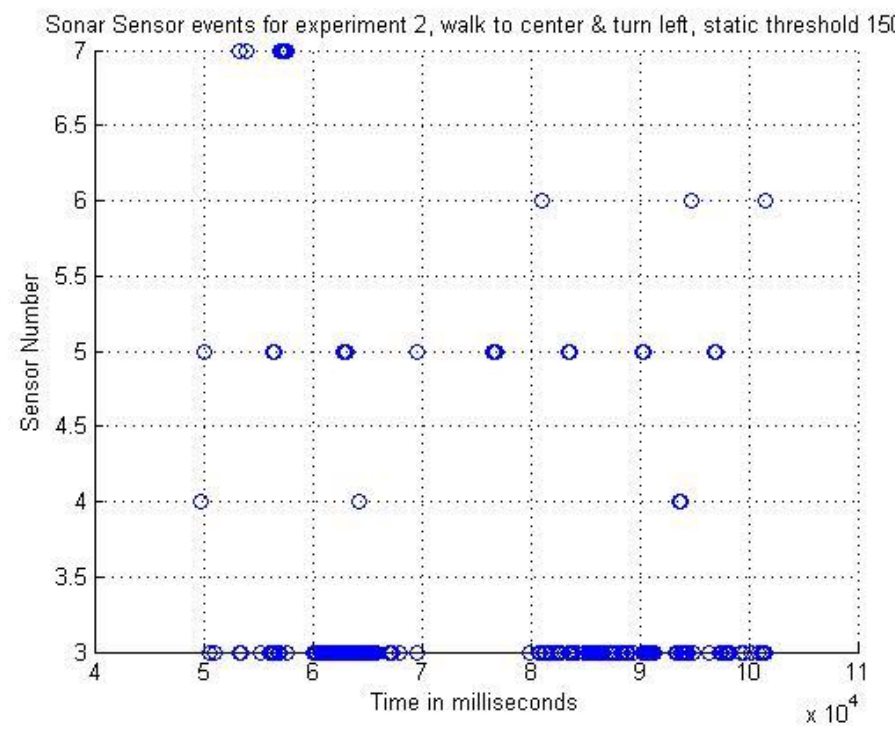

Further analysis needs to be done by averaging 10 samples per second of sonar data to get one value per second, and graphing these results. Then we can rule out the sonar sensors entirely, if necessary. 


\section{Trying to weed out sensor noise with averaging}

The sonar sensors were polled ten times per second. Sometimes the sonar data can be very noisy. Sonar data has been observed with spikes, showing a phantom object very close, or in the opposite case, the object currently in view has suddenly moved back several yards in $1 / 10$ th of a second. Since we know this is impossible, we suspect the sensor is simply reporting echoes and regularly explainable wave cancellation events.

An example of noise in the sonar sensor is below. This is for location 7 , experiment 1 for a duration of 1 second, at a time when we know there was no subject in range. The arithmetic mean for this experiment is 259.23 and we see seven the tuples of raw data below are in the 216-228 range. 12 raw data points are very small. We would expect some wavering like this. However we see a strange "pop" at time: 55.820 where the sensor spikes up to 613 . Does this mean that for $1 / 10$ th of a second the picnic table near this sensor jumped away and then back again?

$15: 45: 55.020,1284763555020,18,1975,4080,224$

$15: 45: 55.120,1284763555120,21,1979,4079,356$

$15: 45: 55.220,1284763555220,21,1971,4079,224$

$15: 45: 55.320,1284763555320,21,1975,4080,216$

$15: 45: 55$. $420,1284763555420,19,1995,4079,224$

$15: 45: 55.520,1284763555520,23,2010,4079,224$

$15: 45: 55.620,1284763555620,15,2002,4080,224$

$15: 45: 55.720,1284763555720,21,2018,4079,356$

$15: 45: 55.820,1284763555820,21,2038,4079,613$

$15: 45: 55.920,1284763555920,19,2044,4079,228$

A new technique must be used to examine raw data values from a sensor that gives noisy readings. We plot the average of the ten readings per second. We would expect to eliminate any spikes or dips such as the 613 seen above. (The arithmetic mean for the 10 samples above is: 288.9). In taking the average we suppress the odd spike to 613 .

A Java program was written to examine the sonar sensor column of the raw data files for each location. This program takes as input some static threshold value. It calculates the average for each second of data for each location and writes rows to a file when any of the calculated values are below the threshold input by the user.

We choose a threshold of 250 and 150 for the following graphs of experiments 1 and 2 . 


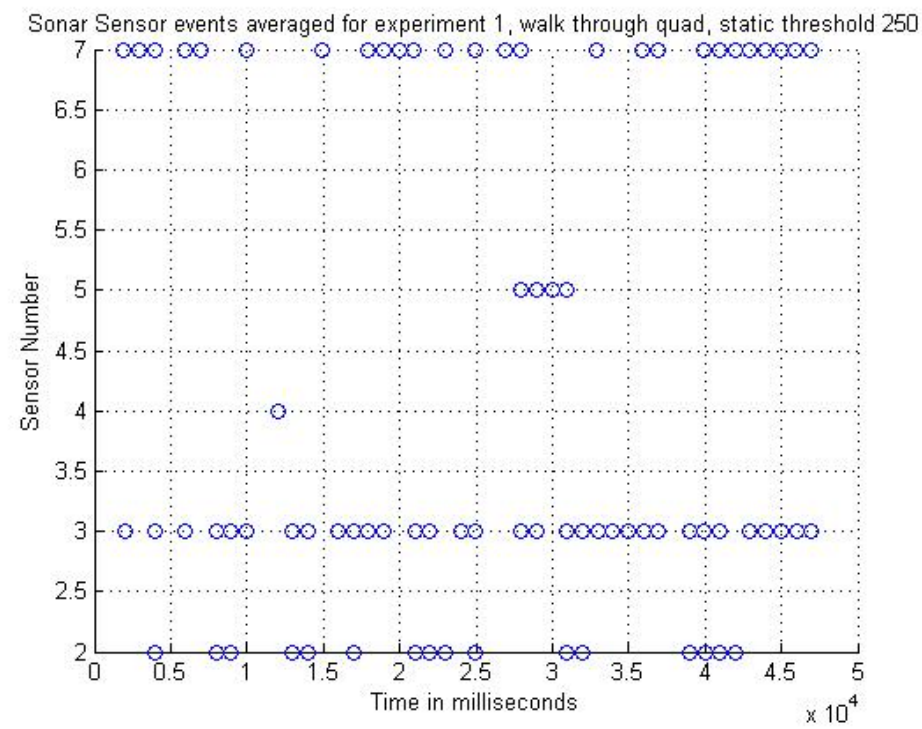

We still see repeated noise patterns in sensors 2,3 and 7 . The walking path of the subject was from $4,3,2,1$ and $1,2,3,4$. This path is clearly seen in the IR motion sensor graphs, but not so here.

Choosing a lower threshold of 150 means the subject has to be right on top of the sensor closer than 1 yard. Graphing the averages per-second at that range gives us fewer plot points but still no definite path for a human subject:

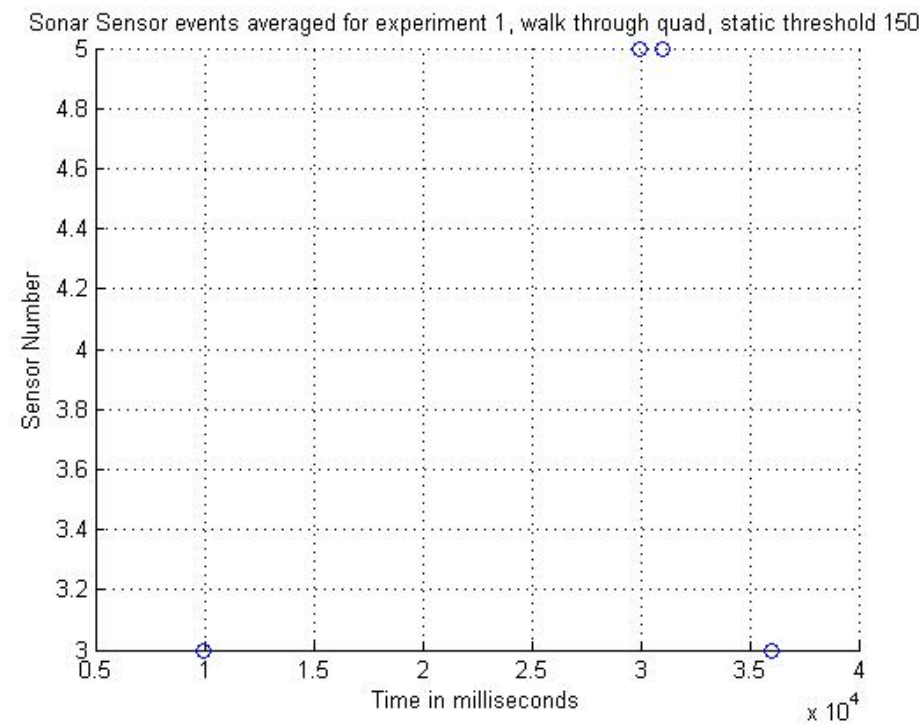

It looks as though we may have caught the subject walking past sensor 3 at the beginning and end of the experiment. Unfortunately that is the only sensor that seems to have worked.

Graphing the averages for experiment 2 with a threshold of 250 show similarly disappointing results: 


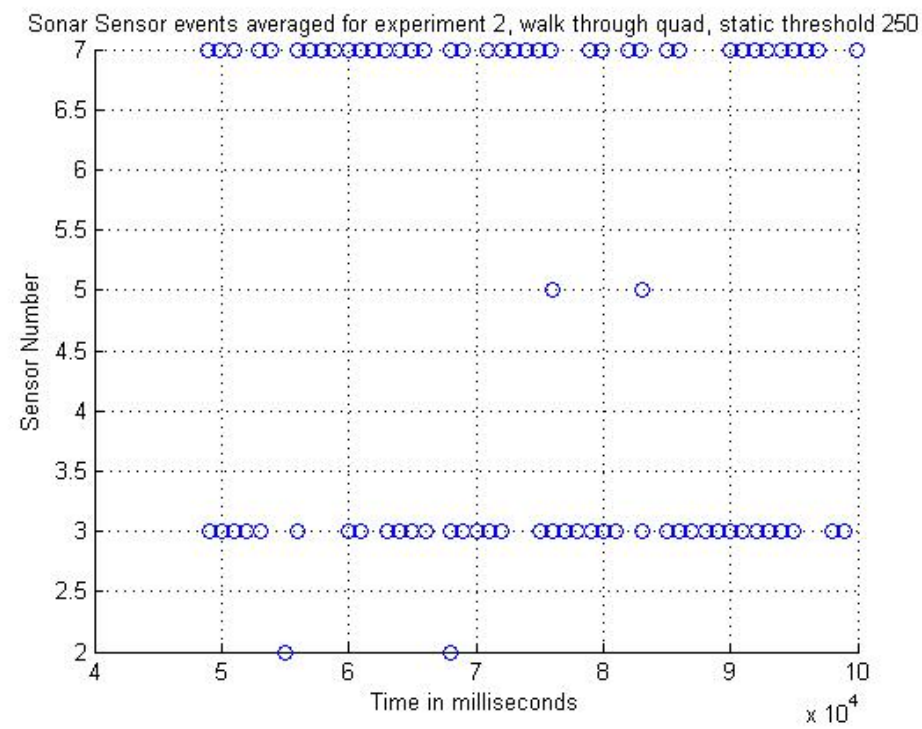

All we can prove by these graphs is that sensors 3 and 7 have regular noise.

Graphing the average per second with a threshold of 150 gives us very few data points to work with at all, although it does get rid of the noise:

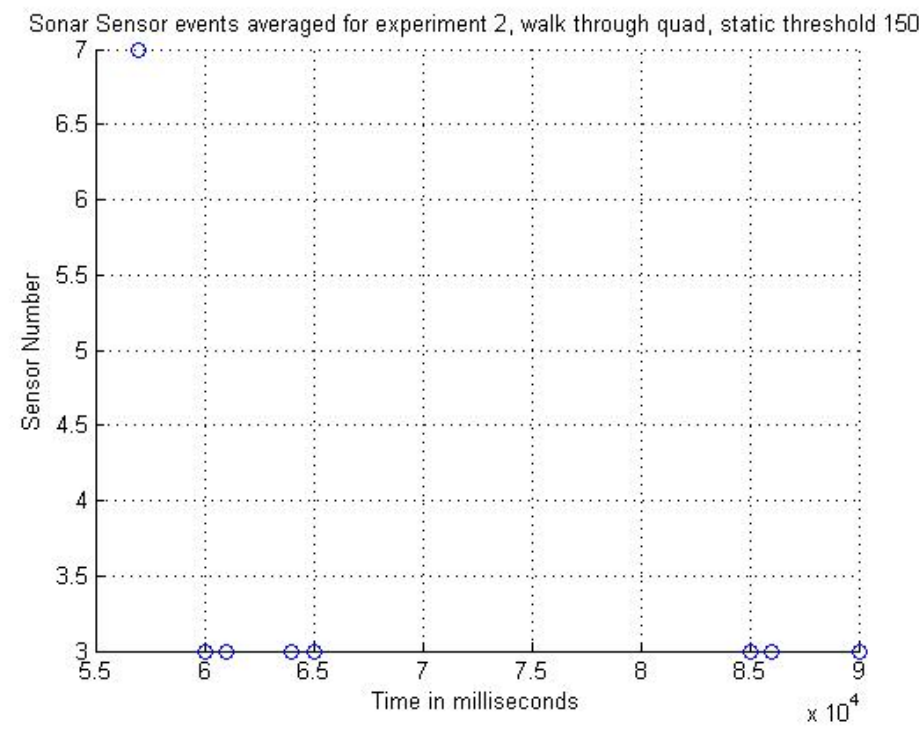

\section{Sonar sensor conclusion}

The sonar sensors were too noisy to product workable data clusters. When no subject was in sight they reported clusters of over-threshold readings. They performed poorly even when noise was taken into account and data was averaged, turning 10 readings per second into 1 workable reading.

One may notice that the sensors were stationary and attempting to see a moving object in our experiments. I believe these sonar distance sensors are meant for a home robotics kit, to keep the robot from bumping into walls. In that case, the sensor itself is moving and the wall being seen is not. It is the reverse situation of how we are using the equipment and it is not well suited for that 
purpose. 


\section{B.4 Light Sensors}

Experiment 1 This is a non-suspicious experiment. Simply walk across the quad and back.

No graph. The light sensors did not report any meaningful data points.

Experiment 2 Walk to center of quad and turn left. Non-suspicious activity.

No graph. The light sensors did not report any meaningful data points.

Experiment 3 Drop a bag at location 6.

No graph. The light sensors did not report any meaningful data points.

Experiment 4 Pick up a bag at location 6.

No graph. The light sensors did not report any meaningful data points.

Experiment 5 Walk to center of quad and loiter around locations 5-7.

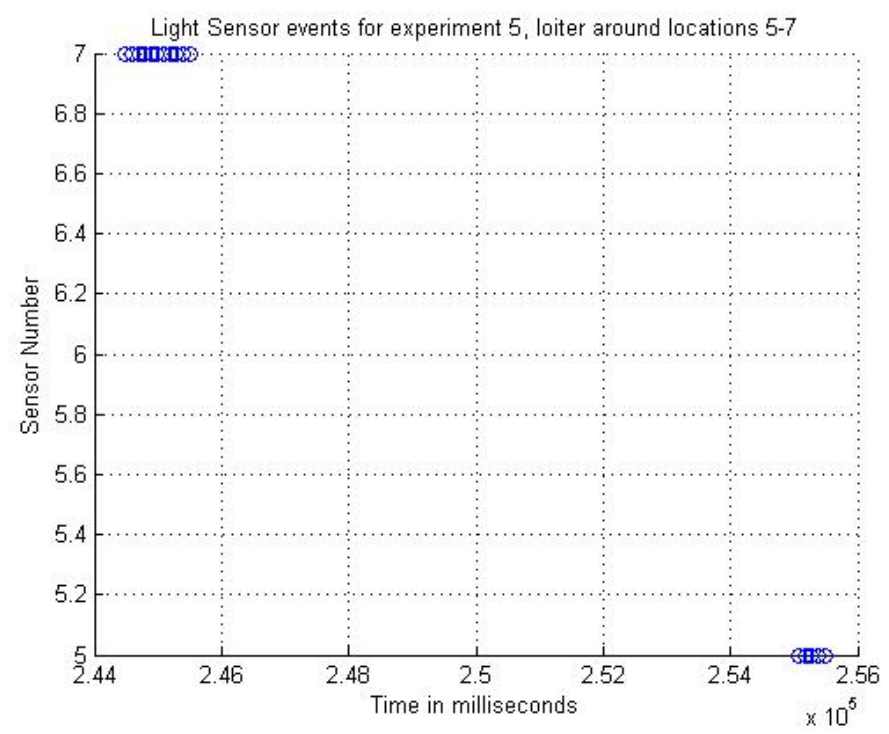

This graph indicates the subject was present around locations 5 and 7 . This can be beneficial when paired up with other sensor readings. It increases the probability of a subject being at a certain location at the reported time when stacked up with data from other sensors.

Experiment 6 Same as experiment 5, with a larger area of loitering around 5-7. 


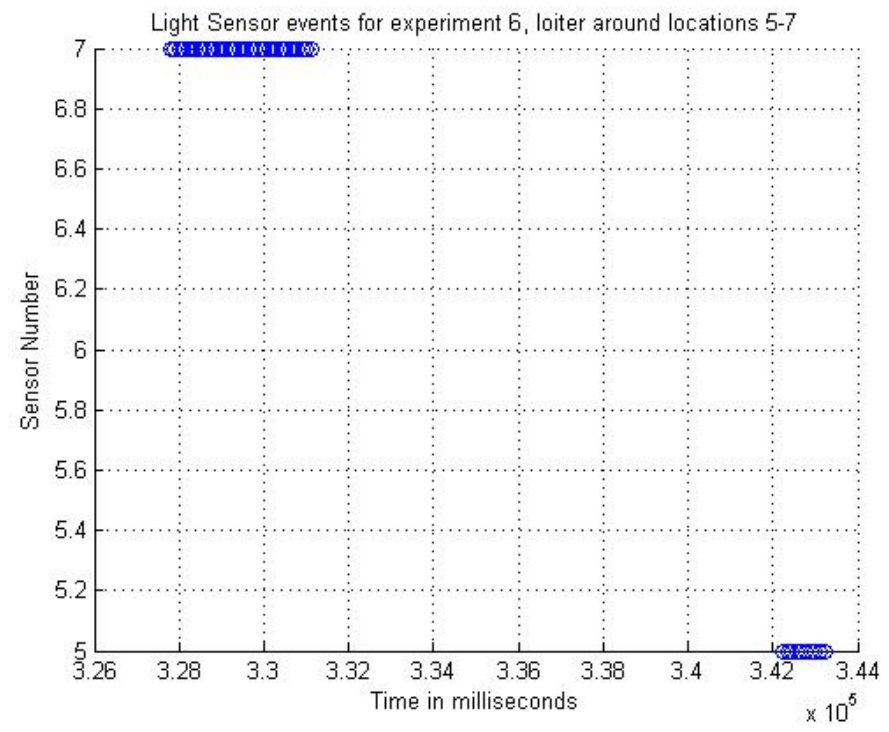

Experiment 7 Walk to center of quad and turn left. Stop at location 5 and dig in the ground, (with 5 shovel hits.)

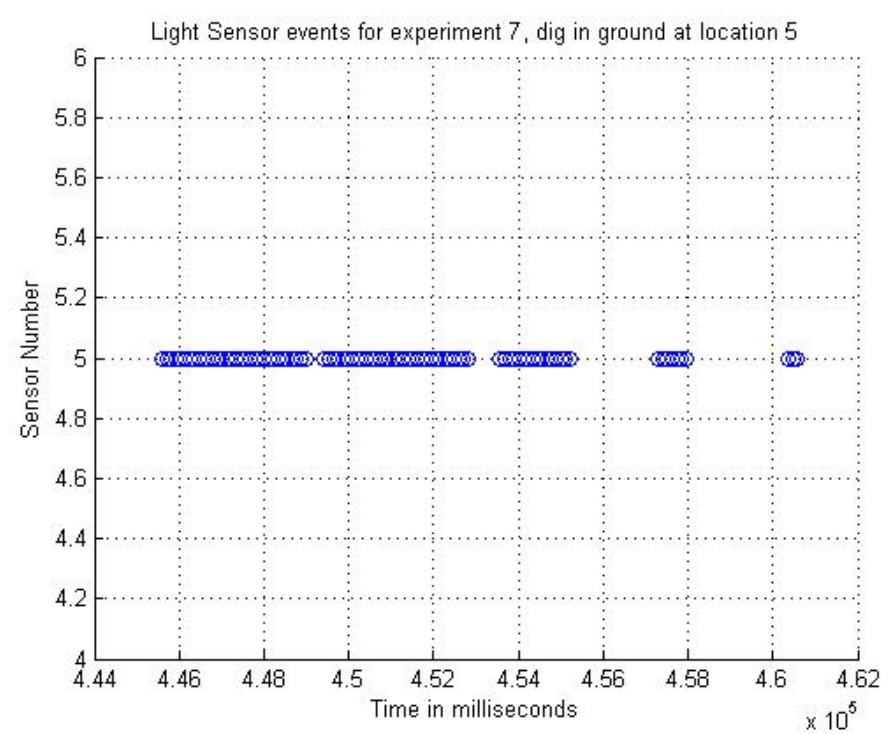

This is fantastic! It shows five distinct clusters representing five distinct shovel digs. There is definitely suspicious activity if the light sensors show clustering; it indicates loitering.

Experiment 8 One subject loiters around locations 5-7. Another subject walks through the quad from top to bottom. 


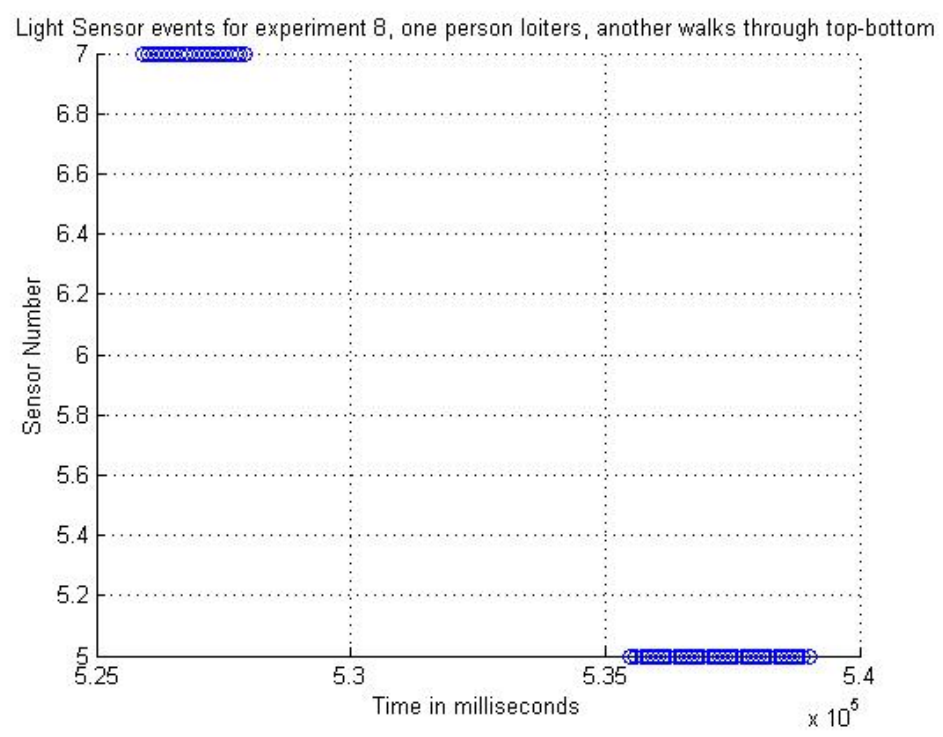

This experiment included suspicious behavior and illustrates detection of loitering at locations 5 and 7 .

Experiment 9 Similar to 8, with one subject loitering around locations 5-7. Another subject walks through the quad from right to left.

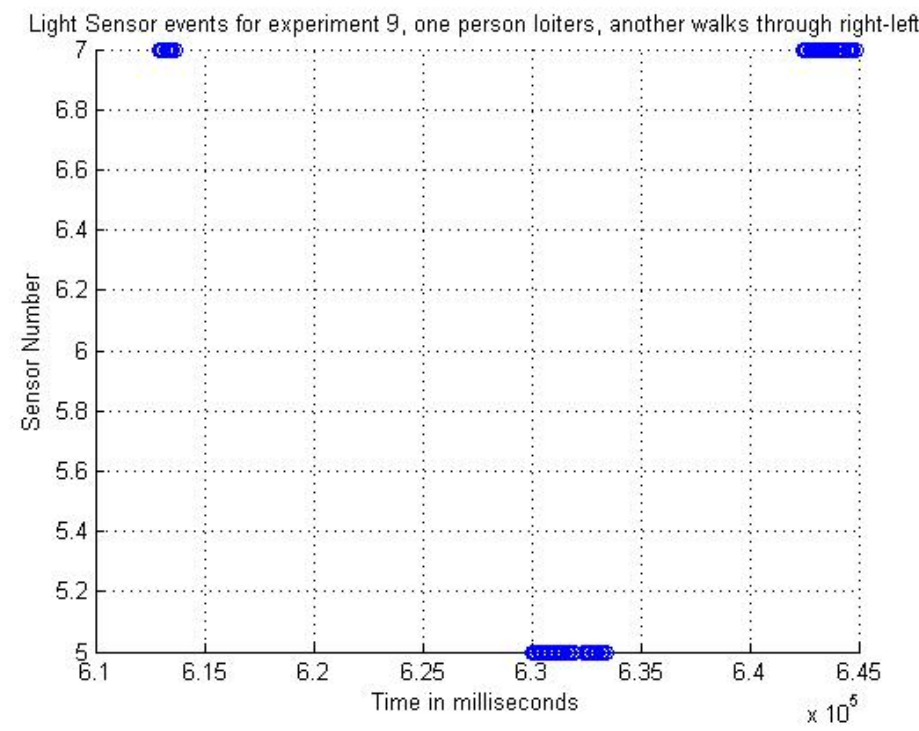

Again more loitering is detected.

Experiment 10 Walk through quad dragging a shovel.

No graph. The light sensors did not report any meaningful data points. 


\section{Appendix $\mathrm{C}$}

\section{False Positives of Sensors}

Some sensors may show activity in the area when there are no human subjects present. There are many reasons why this may occur:

1. Sensor wires blowing in the wind. We had to tape them down.

2. Birds flying by. I did several experiments on the roof of Spanagel Hall at NPS and found seagulls regularly landed on the roof. Sometimes they would take flight and buzz my motion sensors!

3. Leaves and/or bushes blowing nearby. The IR motion sensors my pick up swaying limbs that are nearby.

4. Wind blowing the sensor itself. In this case, the sensor is not detecting motion from a moving subject, it is detecting its own motion.

5. Sensor sensitivity is too high and may need to be adjusted. A higher threshold value may be necessary to use when analyzing data from an over-sensitive sensor.

6. Earthquake? People dropping litter? A paper bag blowing by? A mouse? Paratroopers?

One of the issues with low-cost sensors is that they give binary results. They are either off or on. Frequently home-alarm systems need to be tuned-down so the owner's house cat does not trigger the alarm. Some motion detectors can be set whereby they are only triggered by a larger object such as a person.

False positives may be weeded out through redundancy of sensor coverage [11], however doing so raises the cost of the system. 


\section{Appendix D}

\section{Wireless Sensor Networks}

A wireless sensor network is a distributed system of independent nodes which communicate to local neighbors through radio packets. It is a source-and-sink model where packets are passed down the tree structure to the root node. Each node independently gathers data about its environment with sensors and sends that information to its parent. Message routing protocols exist to recover from individual node failures. A term regularly used is ad-hoc network, which means the network protocol takes care of re-forming the network tree structure regularly, (sometimes every 5 minutes.) Nodes are limited by electrical power, computing power and memory footprint. They must be efficient with resources when deployed in an environment, and therefore try to conserve power as much as possible. Every time the radio is turned on it takes energy. Subsequently the data packets shared between nodes is small, since small data packets consume less energy to send during communication.

A Wireless Sensor Network is characterized by the following:

- distributed system

- many small nodes

- source and sink model

- limited electrical power

- limited computing power

- limited networking packet size

- nodes contain sensors to monitor environment

- ad-hoc networking route protocol

\section{Distributed System}

Many computers communicate through a network towards a common goal. Some examples of distributed computing involve the concerted effort to solve a complex problem; each computer works on a small piece of the computation. In other cases-such as wireless networks-each computer is limited in computational power. Instead they are grouped to monitor an environment by taking regular sensor readings. Individual computers have a very limited view of the entire system. A typical wireless sensor network will have one central computer which processes all of the data sent to it by the nodes forming the network. For example, a vineyard manager might have a PC and a base-station in his office, and a network of a hundred wireless nodes taking temperature readings on 100 acres of vineyard. Each node might monitor a single acre, yet the manager has the entire 
temperature map of the property on his desktop.

\section{Many Small Nodes}

The individual devices manufactured by Crossbow and Memsic are referred to as Motes. A typical mote may be the size of a circuit board on top of two AA batteries. The MicaZ mote is approximately the size of a 1 Euro coin. Each Mote is a low-powered device containing a small processor and a limited amount of memory. In fact, the entire operating system and program for the sensors will sometimes have to fit in under $2 \mathrm{~K}$ of memory. To take advantage of the tiny memory footprint, programmers have developed a language called $\mathrm{NesC}$, a subset of the $\mathrm{C}$ language. The operating system is called TinyOS and is based on Unix and Posix. Small batteries and limited-function chipsets (for example, the Atmel ATmega 128L processor running at 4 megahertz,) contribute to power saving and longevity in the field.

\section{Source and Sink Model}

A base station attaches to a PC which becomes the sink for the network. Each node in the network transmits only to those nearby which it can hear, and a parent is elected for the purpose of sending data to the sink. Each source node takes sensor readings from its immediate environment and creates a small data packet to be transmitted downstream to the sink. In some networks a reduced-function device is placed, whose sole purpose is to act as a networking conduit; it may not take sensor readings at all, focusing its power on transmitting packets downstream instead. The transmitted radio packets must be short, in order to reduce power consumption. Also, when a node is transmitting, it can not simultaneously be receiving on the same antenna, and some messages may be missed or ignored.

\section{Limited Electrical Power}

A node deployed in the field may be expected to run efficiently for a year on the power supplied by two AA batteries. Several strategies come into play, such as sending the device into sleep mode and only performing sensor readings at long intervals. For example, you may not care what the temperature is every 10 seconds. Perhaps a temperature measurement every 10 minutes will suffice. As networked devices become smaller they approach the size of "smart dust." There are already computers the size of a coin. Soon there may be postage stamp sized computers; imagine the label on the spine of a library book is really a networked computer! A flat computer the size of a library book label may require a paper-thin battery, running on 1.5 volts. One may require the book to function as a node in a network for several months before someone comes around to re-charge its battery.

\section{Limited Computing Power}

An 8 or 16 bit chip designed for a calculator is not going to have the speed (or register width) necessary to perform the math operations needed for Elliptic Curve Cryptography. Even if the node was tasked with encryption, it may take so long to perform a calculation that it would essentially be âĂIJbusyâĂİ while many network packets fly by. Missing message transmissions are an unfortunate consequence of wireless sensor networks. Basically the only thing you want your Mote to do is to take sensor data, build a network packet and transmit it downstream. As Motes become smaller the physical space limitation will dictate the complexity of the CPU it contains. 


\section{Limited Networking Packet Size}

A small protocol stack is crucial for conserving energy. Both 802.11 and the emerging ZigBee protocol (not widely used due to the non-GNU-license restrictions) have a small memory footprint here. This contributes to a low battery power cycle. Packets are sent to parent nodes and are passed along the path to the sink node at the root. Nodes close to the root have to handle incoming message packets from many leaf nodes. Therefore having small packets increases efficiency and maintains a tolerable workload for devices trying to conserve energy.

There are certain things you can't do in a limited packet. For example, it is impossible to send long encryption keys. Messages themselves consist of a limited envelope size and a very small data portion. A typical node monitoring weather conditions in a vineyard might send a pair of integer values: Temperature and humidity. I.e. 20 degrees and 75 percent. Messages and transmit-time are limited so there can be no streaming video or large audio files.

\section{Nodes Contain Sensors to Monitor the Environment}

Researchers at Harvard University created devices for a wireless sensor network that were used to monitor a volcano. Each node with fitted with a sub-acoustic microphone to detect low-frequency rumblings in the earth. The network was deployed in a ring around the top of a volcano in Ecuador and reported audio data to a sink at the base of the mountain. The system served as a monitoring and eruption detection device, to warn local residents of imminent danger. Other sensors can be fitted with infra-red motion sensors and may be used to monitor rooms in a building. They can report when people are moving about for security purposes. Motes can be fitted with weather-tracking sensors to monitor a small farm or a large industrial growing operation. Researchers in Australia have used Motes placed $1 \mathrm{~km}$ apart to monitor weather patterns in a large portion of the Australian Outback. There are sensors available that contain accelerometers, gyroscopes and compasses. They can potentially fly a swarm of Unmanned Aerial Vehicles, or a group of robots in a factory. People are finding more and more applications for networked sensors every day.

\section{Ad-hoc Routing Protocol}

Current Motes shipped by Memsic (now running the Mote division of Crossbow) use a self-repairing networking protocol called Xmesh. Generally speaking, the ad-hoc network reforms every 5 minutes. Parent nodes are elected based on their proximity to the sink. It is likely that a node will drop out of the network on occasion due to poor battery, something blocking the antenna, physical damage or code corruption. In this case it can no longer function as a path to the sink and may not be relied on by child nodes wishing to transmit data downstream. Thus, the network re-spawns and only includes nodes that some other node can hear. Pathways are not always the same. This can alleviate bottlenecks due to network configuration, and once a damaged or inoperative node comes back online it can be reincorporated into the network. 


\section{Appendix E}

\section{Prototype sensor network proposal}

A prototype network may have a hierarchy of clusters. For example, a dozen small-scale Motes report to a local Sink Mote. The Sink has more computing power and can perform local analysis on calculations. It also handles backbone traffic to the next level of the hierarchy, namely the Main Sink. This is probably a computer monitored by a soldier, and some software will be written with a GUI and a map to bring up location data when suspicious behavior is detected.

One possible use of a sensor network to detect suspicious behavior might be to monitor a dirt road in Afghanistan and detect terrorists planting IEDs. First we would watch the road and identify normal behavior patterns for that area. Perhaps the road is bumpy and rocky, so the typical speed of travel is $25 \mathrm{mph}$. People generally drive on the road, never off it. The general observed behavior is continuous driving at $25 \mathrm{mph}$ without stopping. People don't want to be stuck out here in the heat of the desert so they tend to travel on through the area unless their car breaks down or gets a flat tire.

Therefore, if we observe a vehicle stopping on the road for a long period of time, we can question the driver's motivation. An array of sensors can detect this loitering behavior and give us clues to what is happening. If our sensor system is able to take a black \& white snapshot and send it quickly through the network, someone at a computer at the Police Department or Military may be able to check it out and make a value-judgment about the situation. Do they observe the driver is just changing a tire or are there several people digging with shovels on the side of the road?

If we don't have a camera-and rely entirely on simple, low-cost sensors-our sensor network has still detected the suspicious behavior or loitering in a place where it is unusual to do so and we have achieved some value from the system. We may have to suffice with an alert from the system, so we know to notify the local MP patrol. Officers on duty can drive by and check it out. The system called attention to a potential trouble spot when observation was needed. Compare this with the situation where an officer may have a random patrol route where they may or may not have been in the area at the time, and you can see the benefit of the system. A system to detect suspicious behavior can direct enforcement patrols more efficiently than perhaps random-route paroling would.

The most likely development of a field-usable prototype will incorporate many types of sensors. We may develop a hierarchy of Motes such that some Motes are devoted to low-cost sensors, some Motes have video and/or audio capability, and some Motes may be placed for the purpose of being a data backbone. Backbone Motes would be the root of a local star cluster, and thus two or more network protocols will be in place. In the event of espionage, some Motes may be developed primarily to detect Jamming and radio interference. Jamming a network is suspicious behavior in itself. Being able to triangulate and alert authorities to the source of jamming signals will be useful in maintaining the health of the implemented system. 
Motes with video capability may only be activated with the network software has determined there is sufficient need to activate a Mote that has the potential to cause a network bandwidth (and packet flooding) issue. However, a 5 to 10 second video may be extremely useful in the field where the closest military personnel is kilometers or hours away. A video may simply be a series of CCD pictures that are compressed and sent over the network. The problem of sending video data through a wireless sensor network is a difficult one, although still do-able. 


\section{Appendix F}

\section{Computer Science Methodology}

"Agile implies being effective and maneuverable."

-Alistair Cockburn, Agile Software development

The software development process that I used in my research assistant position at NPS can best be described as "rapid prototyping" in Software Development lingo. One could also consider it a form of Agile Development. Basically Dr. Rowe-as principal investigator-is "the customer" in development terms, and he expressed his needs and wants with respect to the project goals in weekly meetings.

As stated in Agile Software Development, "A 40-person team won't be as agile as a six-person colocated team." Small teams seem to work best for rapid testing of hypothesis. During my internship position at NPS in summer of 2009 and 2010, we worked in an agile development style, trying to solve problems and work out an experiment design.

For example, part of our project involved mounting a Phidgets gyroscope sensor on the end of a Nerf shotgun toy. This was for an experiment that analyzed how Marines cleared a room; we needed to know where their weapons were pointed. We ordered Phidgets gyroscopes and needed to build a quick Java program to get data from them. This involved going to the manufacturer's web site, downloading their Java API for this sensor, and learning how to interface with the device. Once that was taken care of, there were other problems that came up that needed to be tackled. For example, the gyroscopes did not tell you the attitude of the sensor. They only reported angular rotation in degrees per second for 3 axes. The next task-after learning how to poll data from the sensors-was to integrate the angular rotation data. I had to quickly learn how to implement discrete integration in my program, and modify the existing program to output a file with attitude (positionin-space) data for the Nerf weapon. Although the Nerf weapon and the Phidgets Gyroscope did not play a role in this thesis, it serves as an example of the programming environment for this work. It also shows how simple software solutions were implemented to solve problems quickly as we needed to explore a problem space. The end result of the Nerf weapons experiment can be found in the paper, Visualizing summaries of performance for instructors assessing physical-motion skills [31]

\section{Program Specifics:}

There were several programs written in Java to both get data from the sensors and to analyze it. (see following list) Also, Matlab was an integral part of both Dr. Rowe's analysis and my own. The graphing feature plays heavily in this thesis. Dr. Rowe preferred to program in Matlab, which has a fully functional C-like language built in for analyzing comma separated data files and matrix 
manipulation.

\section{Helper Programs written in the Agile Development style:}

Acceleration.java

This program may turn out to be an entirely separate project rather than an Agile Development quick implementation. A decision was made to halt work on this program and focus on other tasks due to the amount of time it was taking. I was working on integrating the accelerometer data from the Phidget Spatial to get the speed. This was a difficult process. The program is incomplete because the calculations get tricky if the accelerometer is not traveling in a flat, horizontal plane. Once you tilt the device (pitch and roll) the gravity effects need to be calculated. This would have been OK if our accelerometer was on a home-brew robot traveling on a flat floor surface, since it never tilts or moves up an incline. In essence, we discovered how complicated the problem really was by trying to implement it simply and quickly.

Angular.java

Uses Runge-Kutta discrete integration. It takes a data file from a Phidget Spatial, integrates the gyroscope data and provides an output file with pitch values and timestamps. This program works well.

Usage: \$java Angular filename

Output: Orientation.txt

BuildingSearch.java

This program was used in the room-searching tests with the Marines. This program took data from a SpatialPhidget object and a PhidgetInterfaceKit object. It makes an output file with 2 timestamps and all the various sensor data. We used this program to gather data in all the weapons experiments with Rey and Corey.

Usage: \$java BuildingSearch <\# samples per-sec> <run-time-in-minutes> <\#sensors> The $<\#$ sensors $>$ refers to the number of extra sensors attached to the Phidget Interface Kit. We always ran it with 2 here, since we had a sonar sensor and a vibration sensor attached.

Output: phidget_raw.txt

Motiontest.java

This is a simple program that listens for the attachment of a Phidget Interface Kit. When one is connected, it listens for interrupts sent by the Phidget Interface Kit. This happens every time some device (a sensor) reports a change in data. Basically this program was used to see if the sensors were working, and what data they report.

Usage: \$java Motiontest

Output: data to screen

Spatialmotion.java

This is a test program I wrote for the Phidget Spatial. It takes a number of samples per second and a duration in minutes, on the command line. This program catches interrupts from the SpatialPhidget class object. The program calculates an acceptable data rate (some number of milliseconds divisible by 8) and tells the SpatialPhidget to send regular interrupts. Each interrupt contains a SpatialDataListener class object which contains all the data from the Phidget. The program prints out a row each time it gets data. The output file is called: motion.txt. Note: We do not poll the Phidget for data. This program was a little complicated to write because of the sensor listener that had to be set up and interpreted.

Usage: \$java Spatialmotion <\#samples per sec> <\#minutes> 
Output: motion.txt

Rowpicker.java

This program looks a raw data file from a Phidget Interface Kit. You choose a column. It calculates standard deviation on that column, then it goes through the data file and pulls out all the rows with data in that column that are plus-or-minus 2 Standard Deviations. I was using this to detect when something interesting happened, going by the premise that the interesting rows will be the ones with data much different than the base readings from the sensors. This program prints out a file with the interesting rows in it.

Usage: \$java Rowpicker filename column

Output: filtered_rows.txt

Scrubber . java

I wrote this program for Rey. He needed to read a data file and remove the first $n$ number of rows from it. So for example, we can get rid of the first 2 timestamp rows in a data file. The program writes a new file with just the remaining columns of data.

Usage: \$java Scrubber infile outfile num-cols-to-scrub

Output: whatever the user wrote on the command line as âĂIJoutfileâ $\breve{A}$

Sensorfilter java

This reads data at regular intervals (polling) from a Phidget Interface Kit. This is helpful if the sensors are behaving noisy. It uses a recursive exponential filter to eliminate noise from the data. I found the equation in a paper written by a researcher at Lawrence Livermore Labs. This program works well when gathering noisy data from a motion sensor, or a sonar sensor, for example.

Usage: \$java Sensorfilter <\#per-sec> <run-time> <\#sensors>

Output: phidget_raw.txt

Sensortest. java

I wrote this program to sample (poll) a PhidgetInterfaceKit and get data. We have been using this program all summer, in the experiments. It writes a raw data file called: phidget_raw.txt

Usage: \$java Sensortest <\#per-sec> <run-time><\#sensors>

Output: phidget_raw.txt

Spatialtest.java

This is the same thing as the Sensortest.java program, it just reads data from a Phidget Spatial instead of a Phidget Interface Kit. It polls the Phidget, instead of setting up a data rate and a listener, (which is more complicated to program.) Has a few bugs. In the spirit of Agile Development, I abandoned this program and started writing Spatialmotion.java instead. It is important to know when to switch gears.

AzimuthAngle.java

This program compares data files from two Phidget Spatial devices. It matches timestamps and does the angle comparison metric, figuring in pitch.

Usage: \$java AzimuthAngle file1 file2

Output: angle_list.csv

Orientation. java

Takes a file that has been run through Angular.java (a file with orientation angles) and graphically displays pitch and yaw.

To use: Make a test of a gyroscope using Spatialmotion.java. Run the output file through Angu- 
lar.java to get a data file with orientation angles. Then run the new data file with Orientation.java to see what the motion looks like.

This program is currently set up to take values from 0 to 360 . I had previously written it to accept both negative and positive angles. i.e. -90 in the yaw would move it 90 degrees left. Now left is 180 -through- 360 .

Usage: \$java Orientation file1

Output: java Swing on screen

Thresholds.java

The user inputs a number of CSV files corresponding to various locations in the sensor field. Generally these will all be from the same experiment, i.e. Exp. 1, Walking through Quad. The user must also input which column (same in all files) that needs to be analyzed. The program determines the arithmetic mean for each file (corresponding to a particular sensor) and pulls out rows with data points more than a certain number of points away from that mean. For example, if the mean is 2000 and the user inputs 500, all rows $>2500$ and $<1500$ will be pulled.

Usage: java Thresholds column threshold filename(s)

Output: comma separated value file containing tuples selected from one or more files listed on the command line.

Threshold.java

Like the above program, but only operates on a single file at a time. This was written first and then adapted so the program could work on several field location files at once.

ThresholdStatic.java

Similar to Thresholds.java however the arithmetic mean is not calculated. Instead, the user inputs a threshold where only those tuples with data under that threshold are chosen. This was useful in analyzing the sonar sensor data files. 


\section{Appendix G}

\section{Phidgets data acquisition code}

Two programs were written to collect data from the Phidgets Interface Kits. The first (Sensortest.java) takes raw data values from the number of sensors input by the user, at a rate per-second input on the command line. The second program (Sensorfilter.java) uses a recursive exponential filter to smooth out noisy data from sensors. This was useful in black-box testing the sonar sensor.

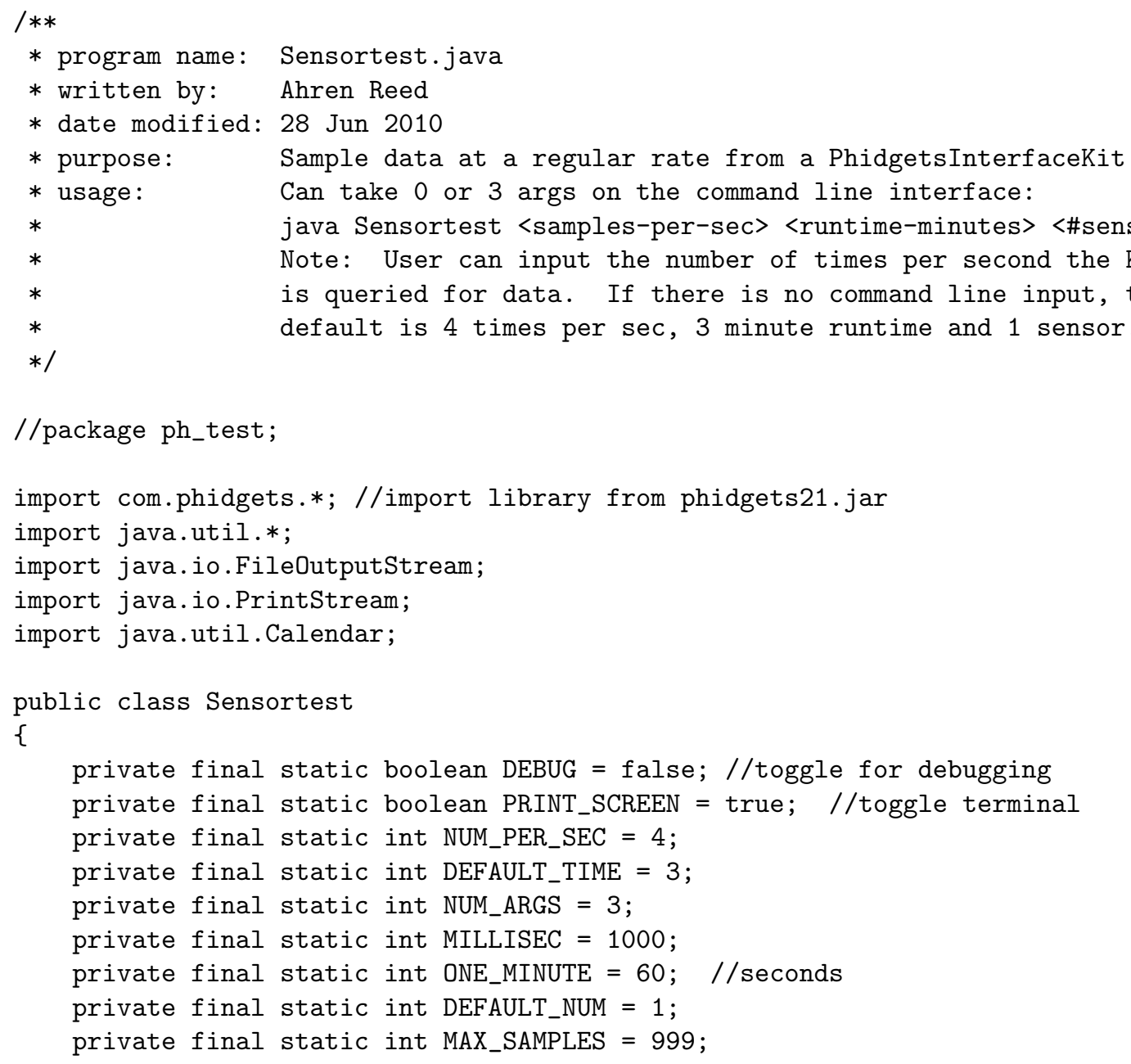




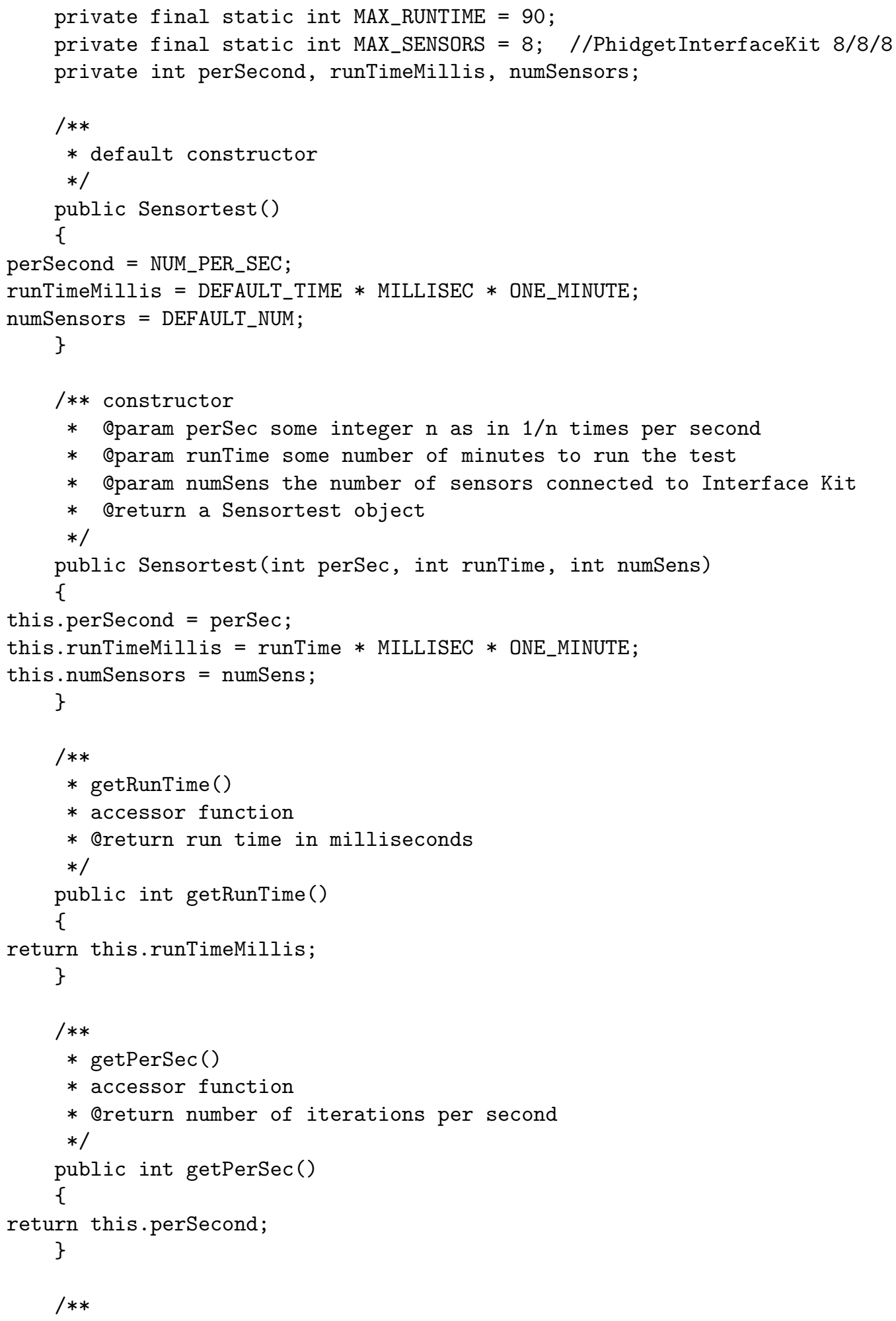




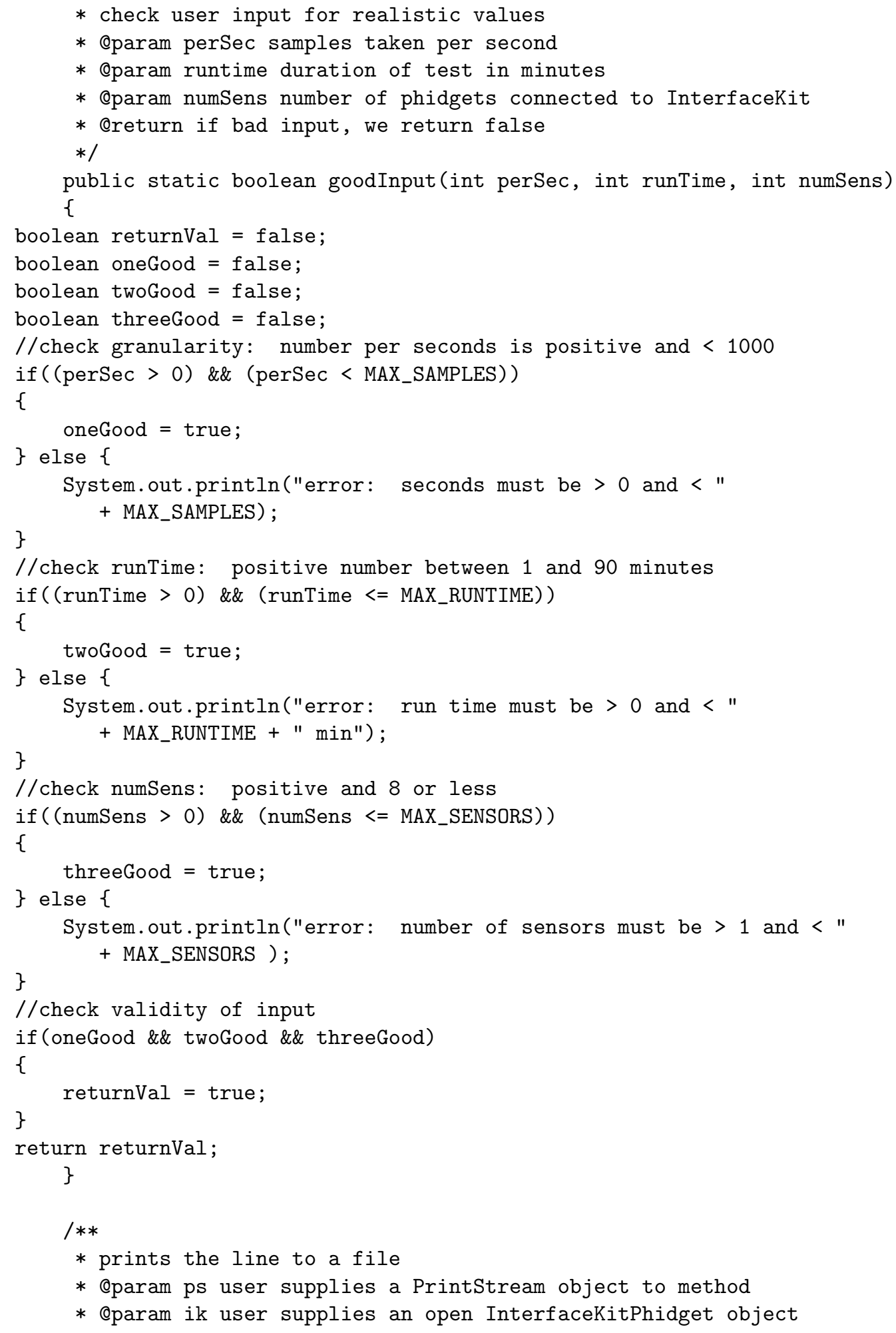




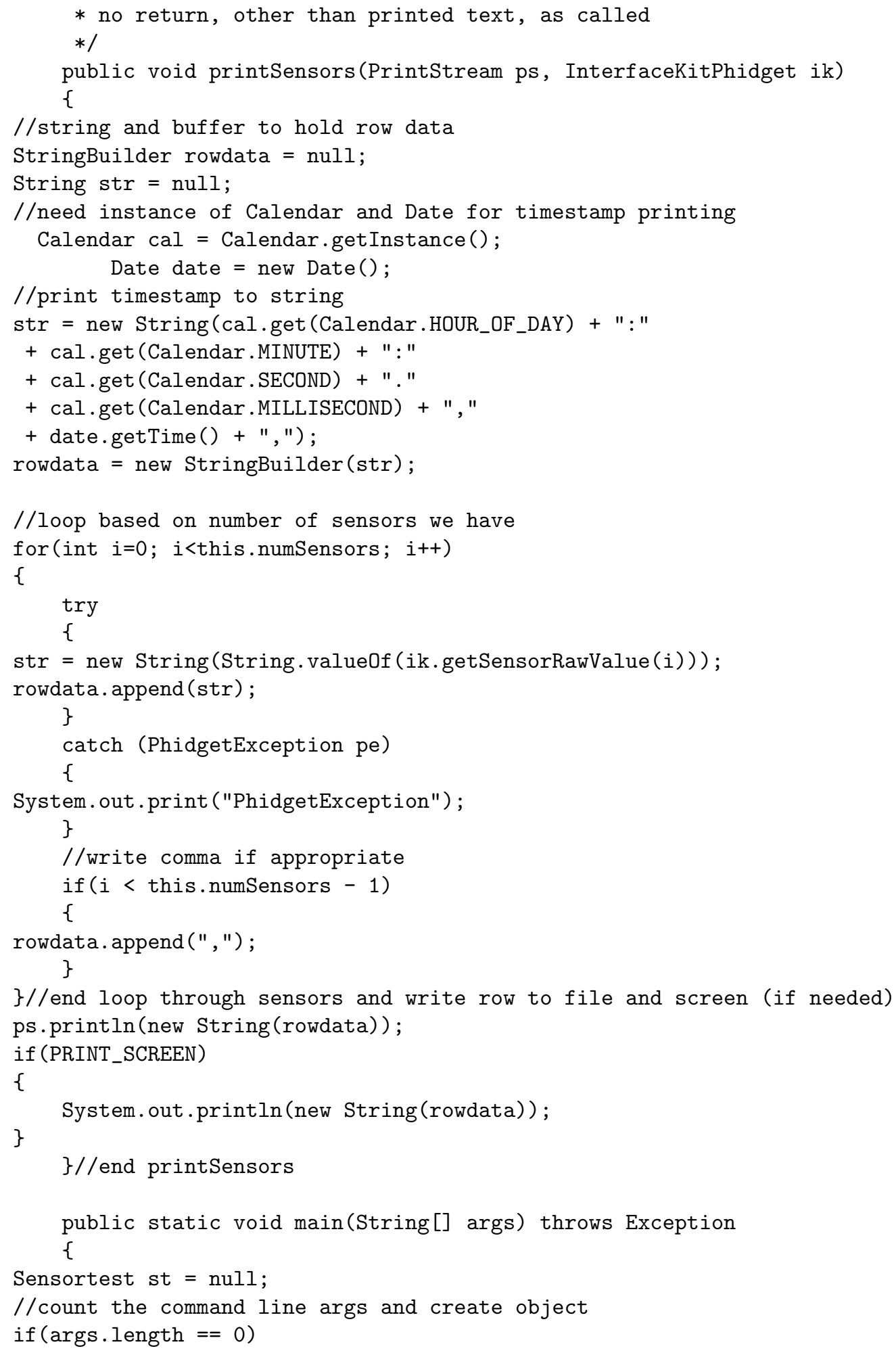




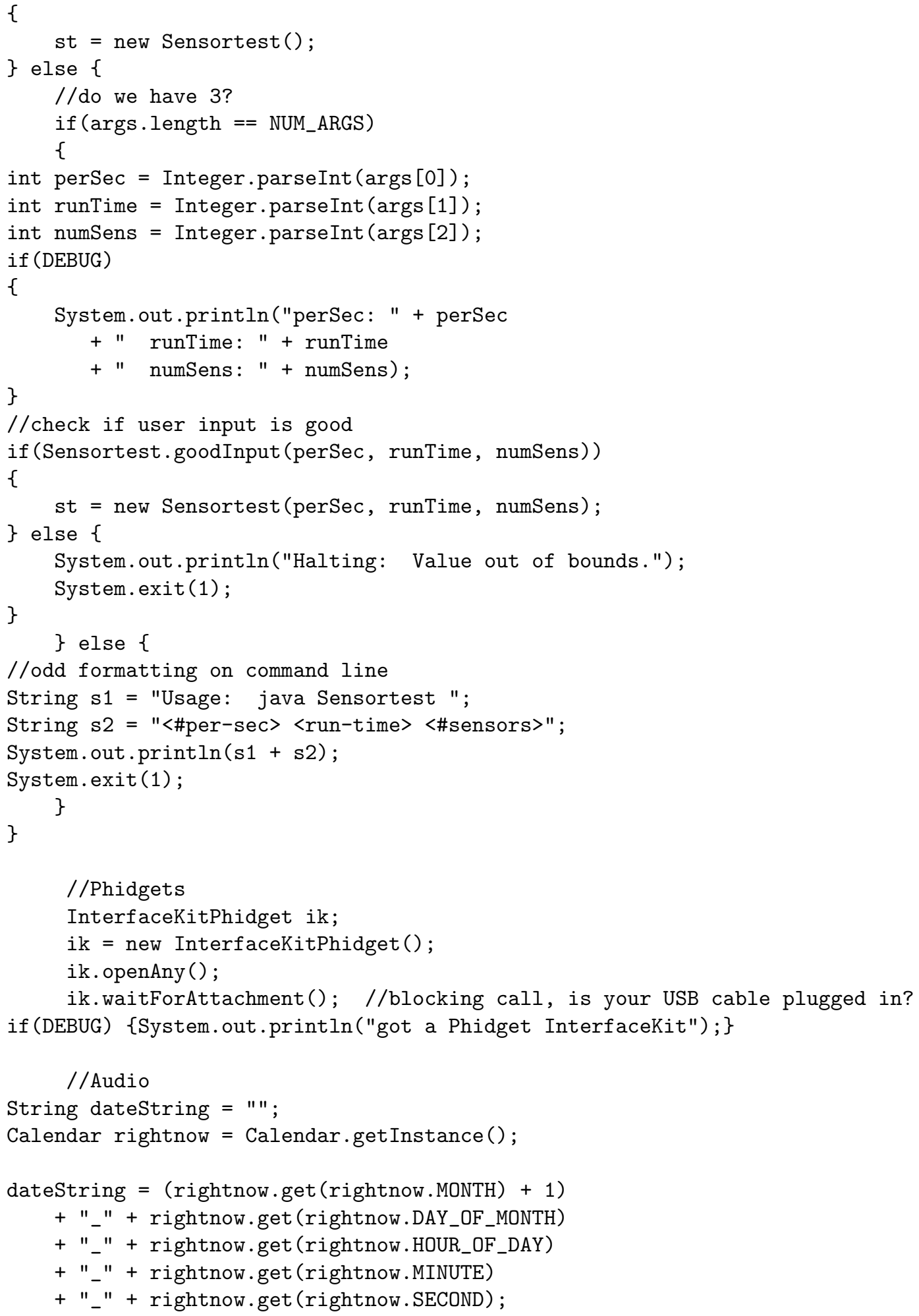




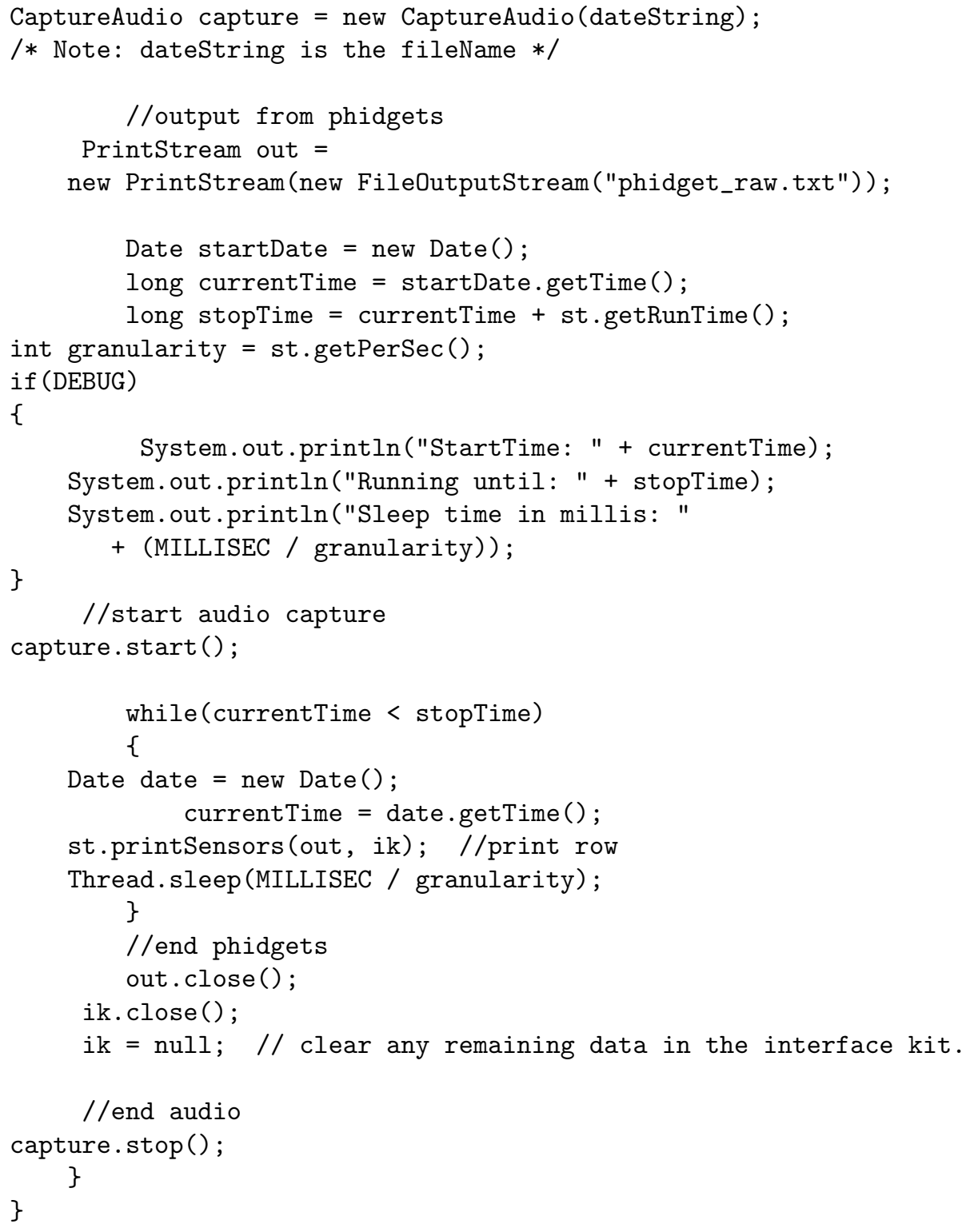




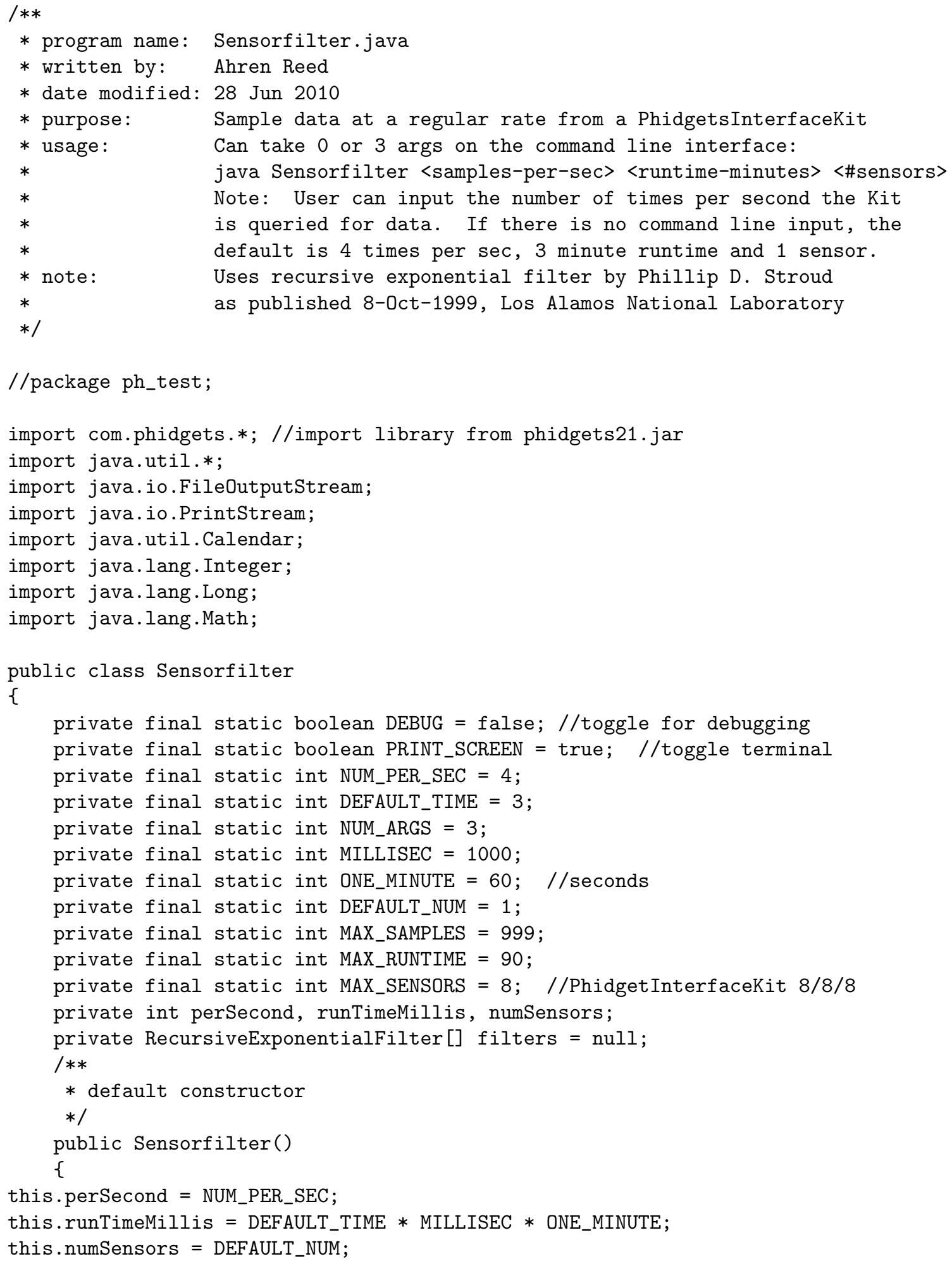




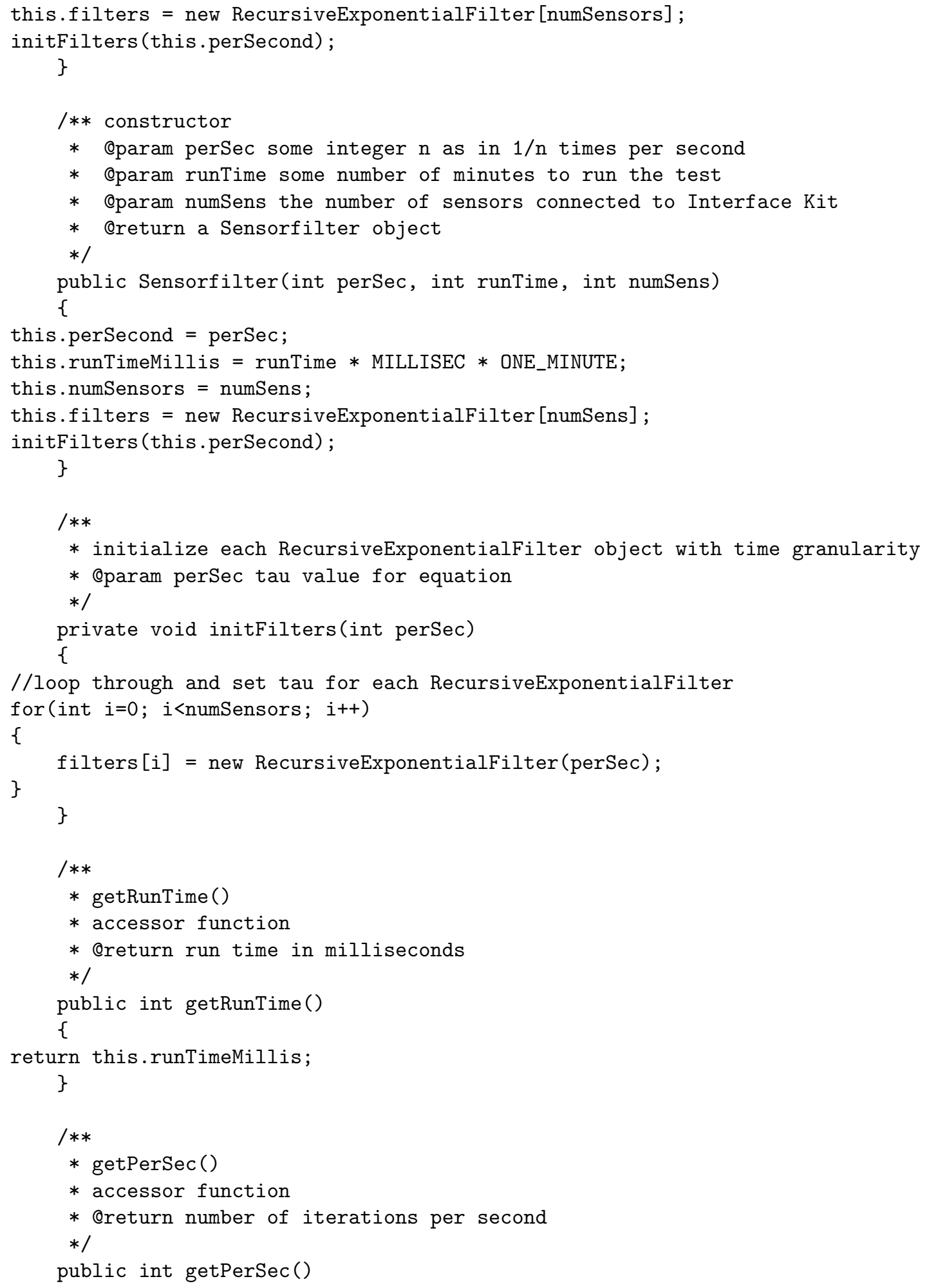




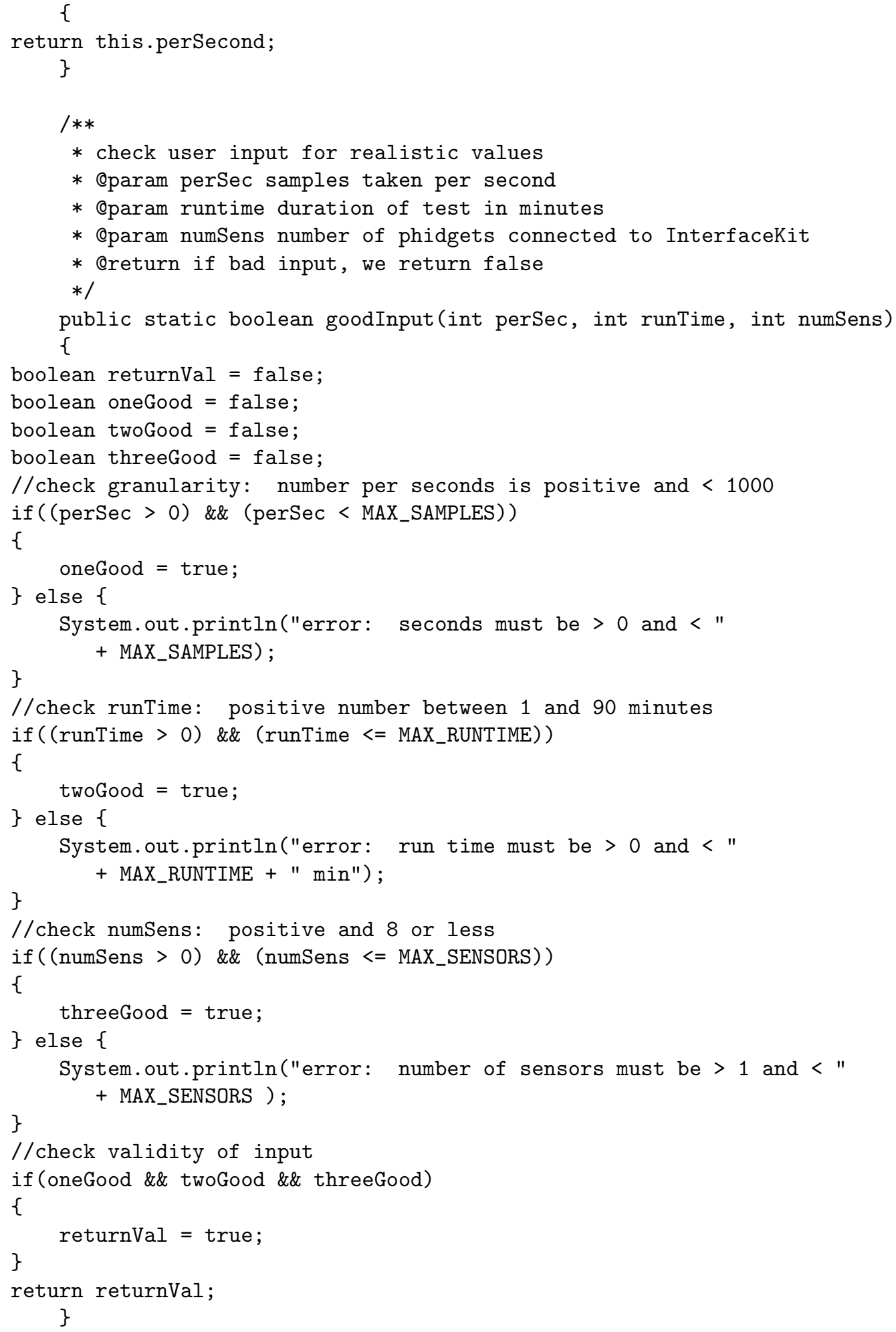




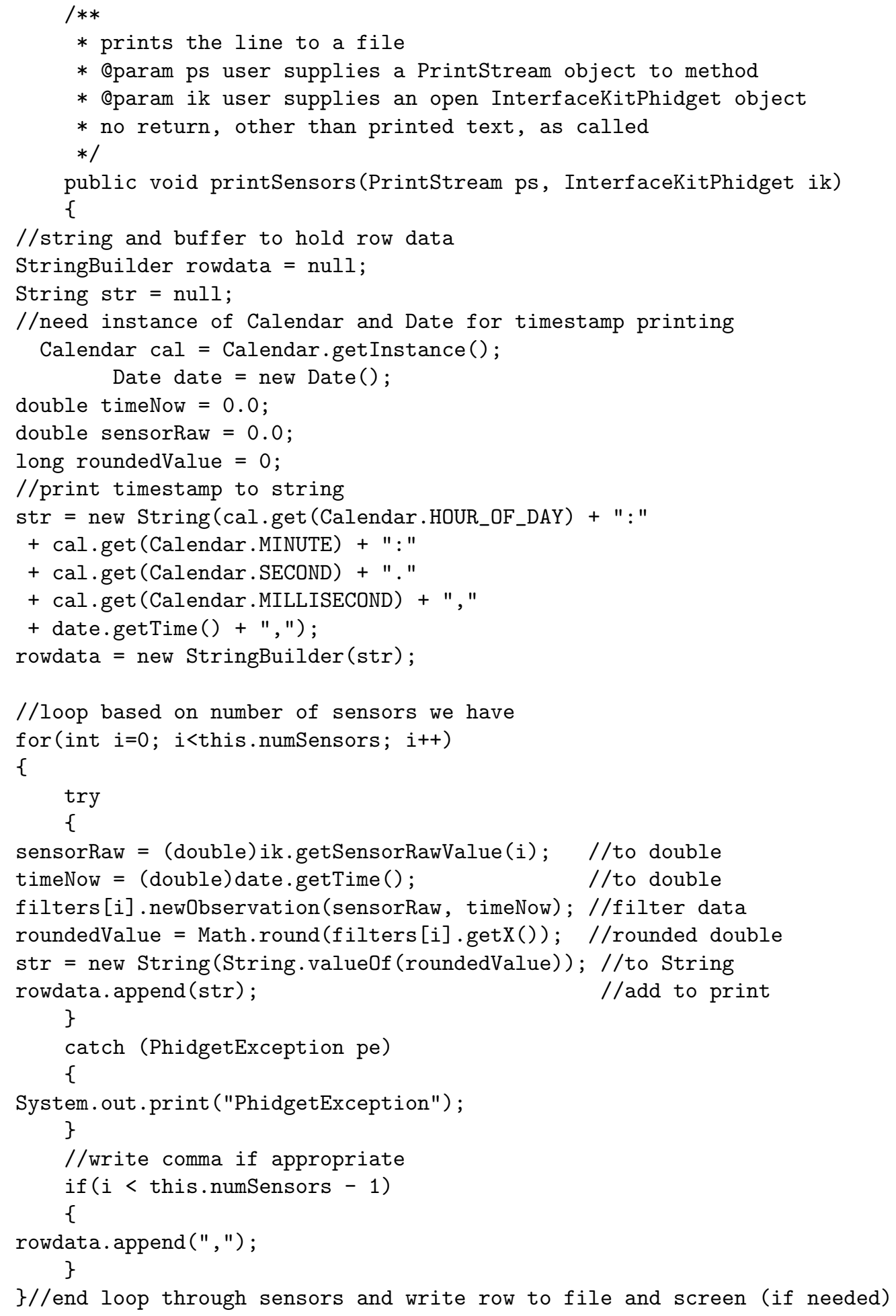




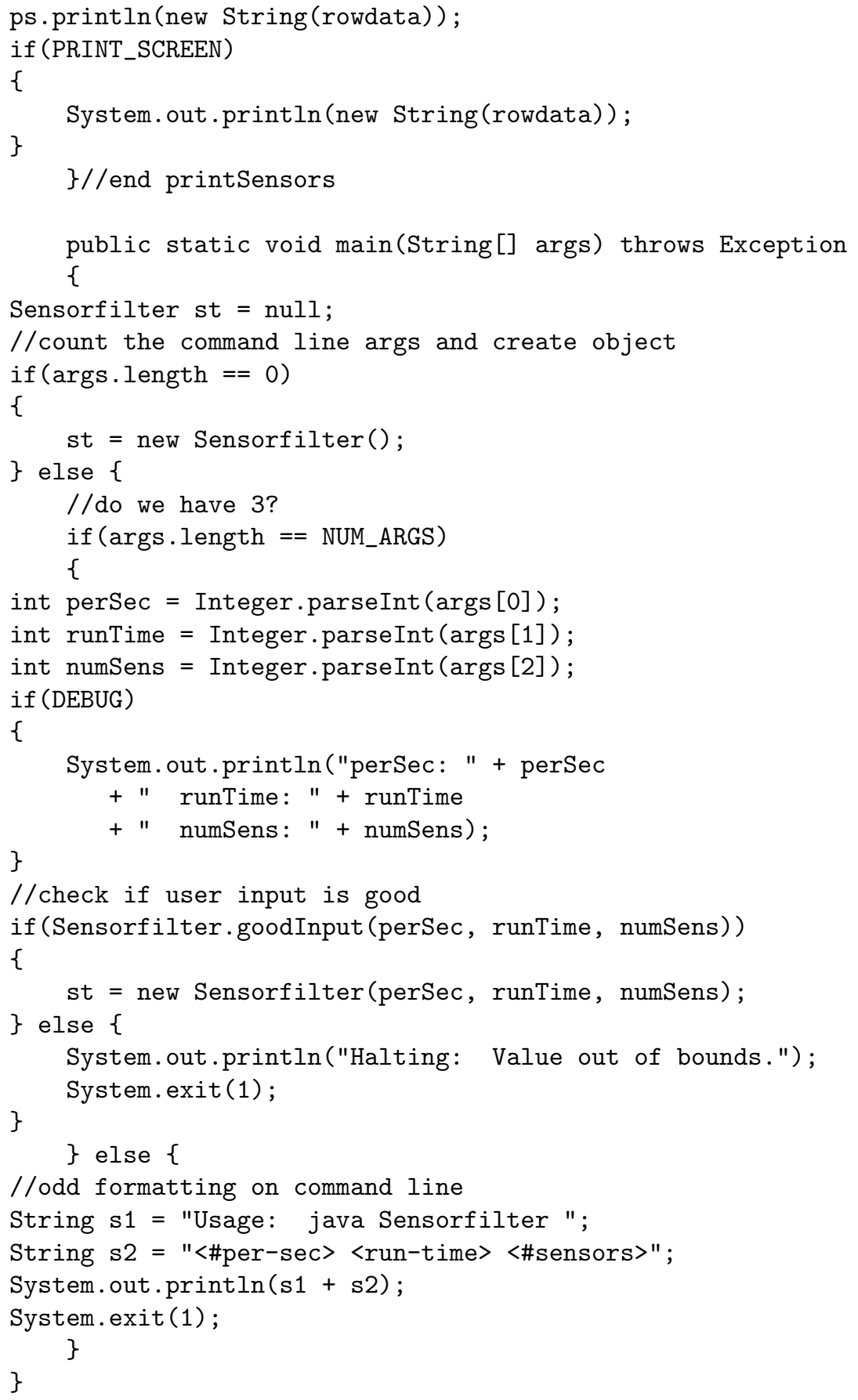


ik.waitForAttachment(); //blocking call, is your USB cable plugged in? if (DEBUG) \{System.out.println("got a Phidget InterfaceKit");\}

//Audio

String dateString = " ";

Calendar rightnow $=$ Calendar .getInstance () ;

dateString $=($ rightnow $\cdot \operatorname{get}($ rightnow $\cdot$ MONTH $)+1)$

+ "_" + rightnow.get (rightnow.DAY_OF_MONTH)

+ "_" + rightnow.get (rightnow.HOUR_OF_DAY)

+ "_" + rightnow.get (rightnow.MINUTE)

+ "_" + rightnow.get (rightnow.SECOND);

CaptureAudio capture $=$ new CaptureAudio(dateString);

/* Note: dateString is the fileName */

//output from phidgets

PrintStream out $=$

new PrintStream(new FileOutputStream("phidget_raw.txt"));

Date startDate $=$ new Date () ;

long currentTime = startDate.getTime ();

long stopTime $=$ currentTime + st.getRunTime () ;

int granularity = st.getPerSec();

if (DEBUG)

\{

System.out.println("StartTime: " + currentTime);

System.out.println("Running until: " + stopTime);

System.out.println("Sleep time in millis: "

\}

+ (MILLISEC / granularity));

//start audio capture

capture.start();

while (currentTime < stopTime)

\{

Date date $=$ new $\operatorname{Date}()$;

currentTime $=$ date.getTime ();

st.printSensors(out, ik); //print row

Thread.sleep(MILLISEC / granularity);

\}

//end phidgets

out.close ();

ik.close ();

$i k=$ null; // clear any remaining data in the interface kit.

//end audio

capture.stop(); 


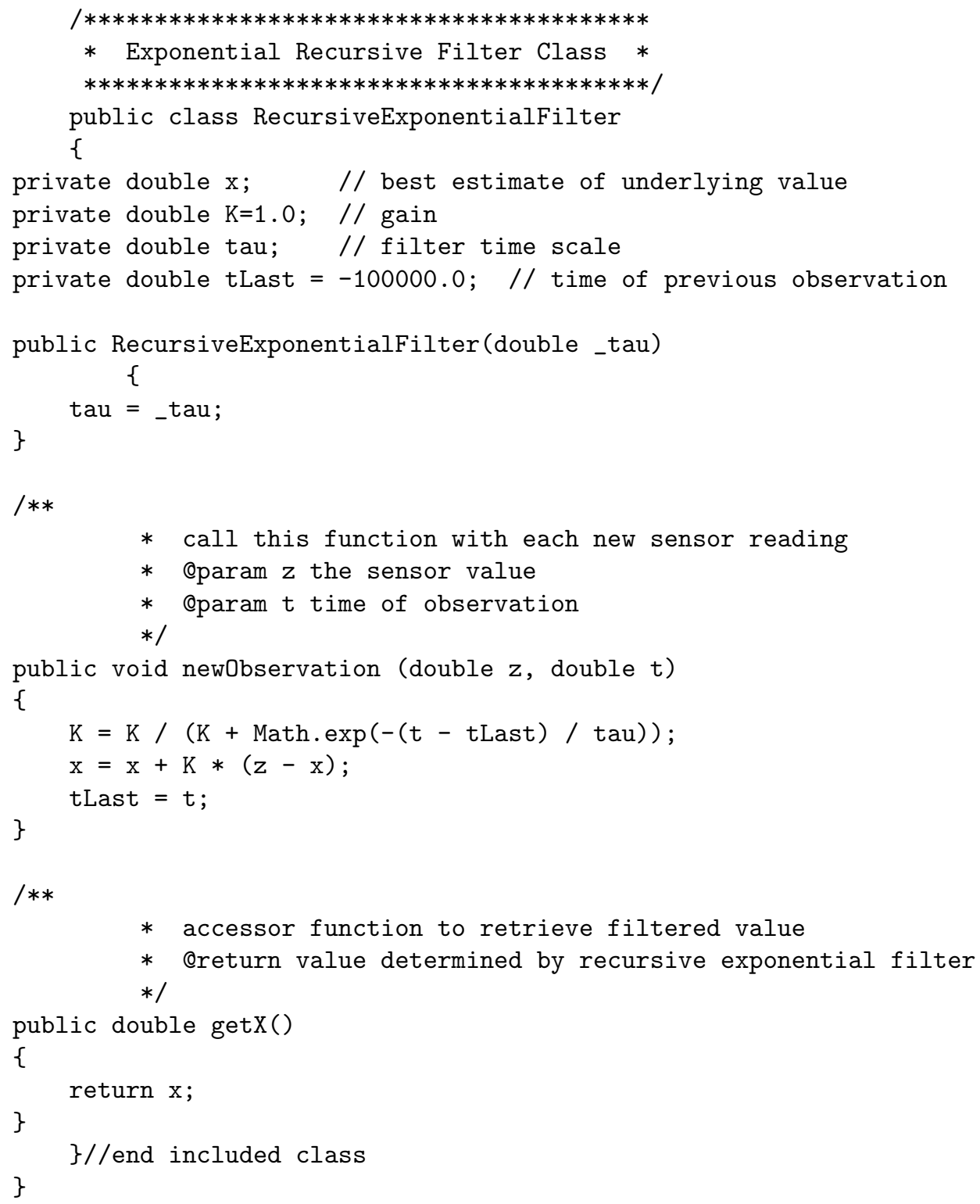

